\title{
Teoria de G-índice e grau de aplicações G-equivariantes
}



SERVIÇO DE PÓS-GRADUAÇÃO DO ICMC-USP

Data do Depósito: 08 de Abril de 2010

Assinatura:

\title{
Teoria de G-índice e grau de aplicações G-equivariantes
}

\author{
Norbil Leodan Cordova Neyra
}

Orientadora: Profa. Dra. Denise de Mattos

Dissertação apresentada ao Instituto de Ciências Matemáticas e de Computação - ICMC-USP, como parte dos requisitos para obtenção do título de Mestre em Ciências - Matemática.

USP - São Carlos

Abril/ 2010 

Eu não acho que eu seja de interesse público, eu não falo isso por causa da minha privacidade, não tenho nada a esconder. Só acho que o público não deve se interessar por mim. Jornais deveriam ter mais discernimento sobre o que publicar, deveriam ter mais requinte. Até onde eu sei, não ofereço nada que acrescente à vida dos leitores. Publiquei meus achados. É isto que ofereço ao público.

Grigori Yakovlevich Perelman 



\section{Agradecimentos}

Inicialmente, gostaria de agradecer a Deus e à minha família, que sempre me deram força, coragem e apoio incondicional para eu seguir em frente, mesmo nas horas mais difíceis. Além disso, pela privação que passaram meus pais, para que eu tivesse a oportunidade de seguir com meus estudos. Amo demais todos vocês.

Agradeço também a uma pessoa muito especial que faz parte da minha vida, Patrícia, por sempre estar comigo e ter me ajudado em todos os sentidos para que este trabalho fosse realizado.

É com muita admiração e respeito que agradeço à minha orientadora Dra. Denise de Mattos e ao professor Dr. Edivaldo Lopes Dos Santos por terem acreditado em mim e pelo tempo e ajuda oferecida no decorrer deste período. Os ensinamentos, dedicação paciência e seriedade profissional deles foram os fatores que conduziram este trabalho. Nada disso teria acontecido sem vocês, muito obrigado!

Agradeço à FAPESP pelo apoio financeiro a este trabalho.

Agradeço a todos os meus professores que fizeram parte da minha vida acadêmica e contribuíram na minha formação como matemático. Em especial, à professora Dra. Márcia Cristina Anderson B. Federson, pelo apoio desde o início desta longa jornada.

Agradeço a todos os meus amigos pela amizade, pelo auxílio e por todos os momentos inesquecíveis que passamos. Em especial, à turma de peruanos pelas horas que curtimos jogando futebol.

À Ana Paula e à Laura, pela atenção que me deram durante todo este período.

Enfim, a todos que colaboraram de alguma forma com este trabalho.

Muito obrigado!! 



\section{Resumo}

Antes da publicação do trabalho "An ideal-valued cohomological index theory with applications to Borsuk-Ulam and Bourgin-Yang theorems"de Fadell e Husseini [20], haviam sido apenas considerados índices numéricos de $G$-espaços, nos casos $G=\mathbb{Z}_{2}$ e $G$ um grupo finito. No entanto, tais índices numéricos são obviamente insuficientes no caso de grupos mais complexos, como por exemplo a 1-esfera $S^{1}$. Neste contexto, Fadell e Husseini introduziram o chamado Indice cohomológico de valor ideal: a cada $G$-espaço $X$ paracompacto, eles associaram um ideal $\operatorname{Ind}^{G}(X ; \mathbb{K})$ do anel de cohomología $\check{H}^{*}(B G ; \mathbb{K})$, onde a cohomologia de Čech $\check{H}^{*}$ é considerada com coeficientes em um corpo $\mathbb{K}$ e $B G$ é o espaço classificante do grupo G. Além disso, Fadell e Husseini associaram a este ideal o Índice cohomológico de valor numérico, o qual é definido como sendo a dimensão do $\mathbb{K}$-espaço vetorial obtido do quociente entre o anel $\check{H}^{*}(B G ; \mathbb{K})$ e o ideal $\operatorname{Ind}^{G}(X ; \mathbb{K})$. O objetivo principal deste trabalho é apresentar um estudo detalhado deste índice e utilizá-lo no estudo dos resultados sobre grau de aplicações $G$-equivariantes provados por Hara em "The degree of equivariant maps"[24].

Palavras chaves: $G$-espaços, aplicações $G$-equivariante, espaços classificantes, $G$-índice, cohomologia de Čech. 



\section{Abstract}

Before the appearance of the paper "An ideal-valued cohomological index theory with applications to Borsuk-Ulam and Bourgin-Yang theorems"of Fadell and Husseini [20], had been considered numerical indices of $G$-spaces, when $G=\mathbb{Z}_{2}$ and when $G$ is a finite group. However, such numerical indices are obviously insufficient in the case of groups more complexes, for example, $G=S^{1}$. In this context Fadell and Husseini, introduced the called valued-ideal cohomological index: to every paracompact $G$-space $X$ they associated an ideal $\operatorname{Ind}^{G}(X, \mathbb{K})$ of the cohomology ring $\check{H}^{*}(B G ; \mathbb{K})$, where the Čech cohomology $\check{H}^{*}$ is considered with coefficients in a field $\mathbb{K}$ and $B G$ is the classifying space of the group $G$. Moreover, they associated to this ideal the numerical valued cohomological index, that is, the dimension of $\mathbb{K}$-vector space obtained by the quotient between the $\operatorname{ring} \check{H}^{*}(B G ; \mathbb{K})$ and the ideal $\operatorname{Ind}^{G}(X, \mathbb{K})$. The main objective of this work is to present a detailed study of this index and use such index on the study of results on degree of equivariant maps proved by Hara in his paper "The degree of equivariant maps"[24].

keywords: $G$-spaces, $G$-equivariant maps, classifying spaces, $G$-índex, Čech cohomology. 

Introdução $\quad$ v

1 Cohomologia de Čech 1

$1.1 \quad$ Limite direto . . . . . . . . . . . . 1

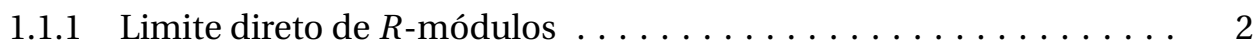

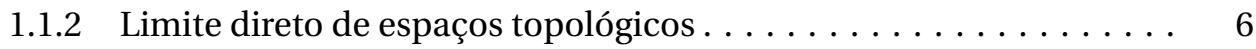

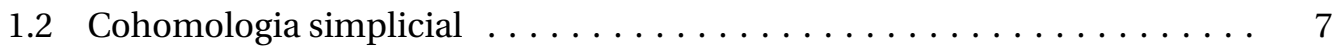

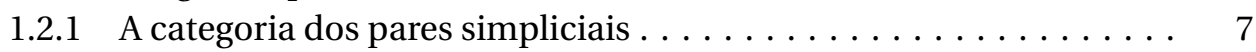

1.2.2 Os $R$-módulos de cohomologia simplicial relativos $\ldots \ldots \ldots \ldots 9$

1.3 Os R-módulos de cohomologia de Čech relativos . . . . . . . . . . . 11

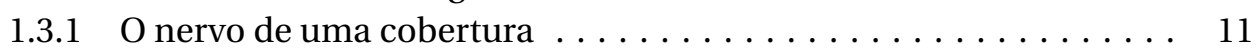

1.3.2 O Homomorfismo induzido em Cohomologia de Čech . . . . . . . 15

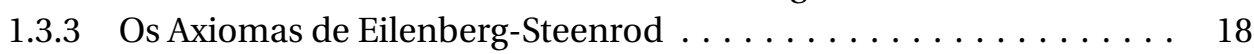

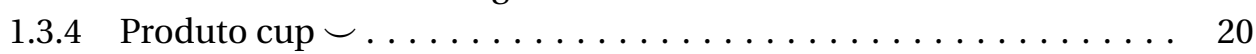

2 Grupos de transformações compactos $\quad 23$

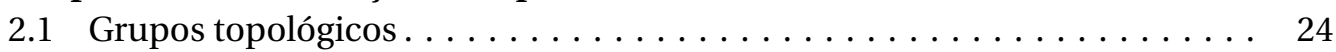

$2.2 G$-espaços e aplicações $G$-equivariantes $\ldots \ldots \ldots \ldots \ldots \ldots \ldots \ldots \ldots \ldots \ldots$

2.2.1 A categoria G-Top ${ }_{p c}^{2}: G$-pares paracompactos $\ldots \ldots \ldots \ldots \ldots 31$

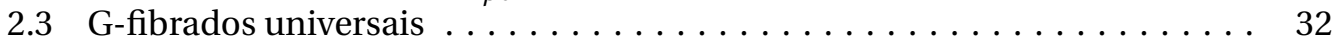

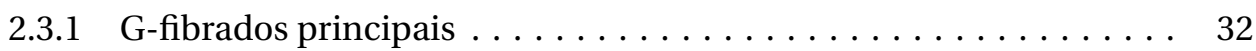

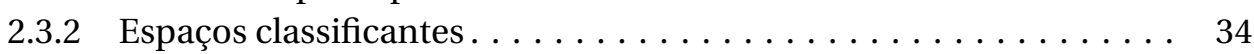

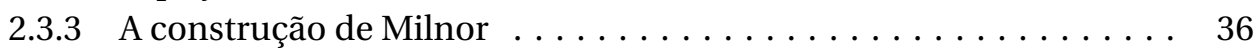

2.3.4 A Construção de Borel $\ldots \ldots \ldots \ldots \ldots \ldots \ldots \ldots \ldots \ldots \ldots$

3 Índice cohomológico de um G-espaço: G-índice 43

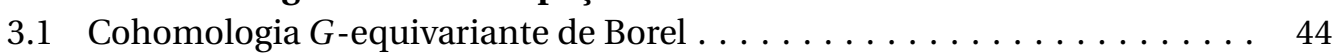

3.1.1 Estrutura de módulo graduado sobre $\check{H}^{*}(B G ; R) \ldots \ldots \ldots \ldots 49$ 
3.2 G-índice cohomológico de Fadell e Husseini $\ldots \ldots \ldots \ldots \ldots \ldots \ldots$

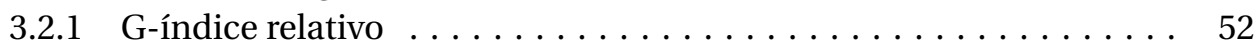

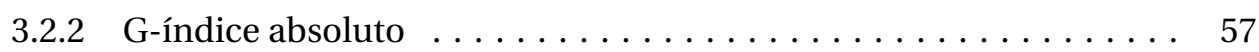

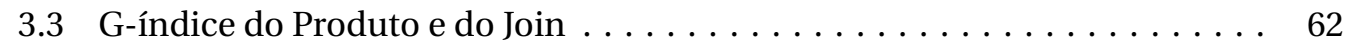

3.4 Casos Particulares de G-índices absolutos $\ldots \ldots \ldots \ldots \ldots \ldots \ldots \ldots 6$

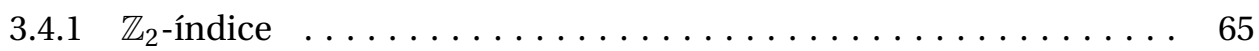

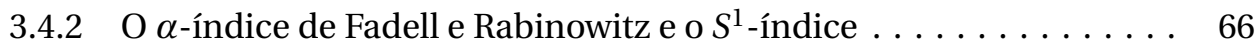

3.4.3 O $O(k)$-índice de uma Variedade de Stiefel $\ldots \ldots \ldots \ldots \ldots \ldots 67$

4 Grau de Aplicações equivariantes $\quad 71$

4.1 O Homomorfismo transfer de Gonçalves e

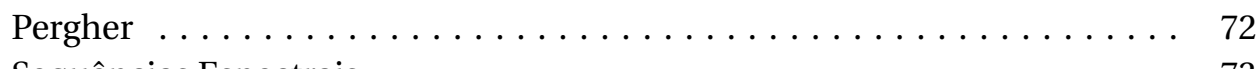

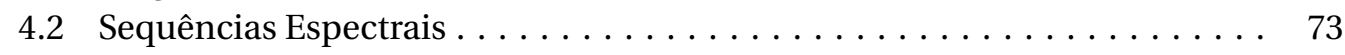

4.3 Integração ao Longo da Fibra $\ldots \ldots \ldots \ldots \ldots \ldots \ldots \ldots \ldots \ldots \ldots \ldots$

4.4 Relação entre o Grau e o G-índice de Fadell e Husseini . . . . . . . . . . . . 77

$\begin{array}{lr}\text { A Fatos Algébricos e Topológicos } & \mathbf{8 3}\end{array}$

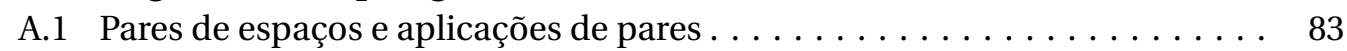

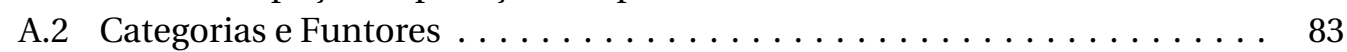

A.3 A Categoria dos Pares Paracompactos: Top To $_{p c}^{2} \ldots \ldots \ldots \ldots \ldots \ldots$

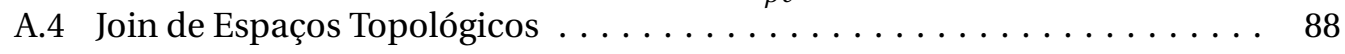

$\begin{array}{lr}\text { Referências Bibliográficas } & 93\end{array}$ 
Vamos estabelecer a nossa terminologia e convenções de notação

$\begin{array}{ll}\cong & \text { isomorfismo algébrico/ bijeção/ homotopia } \\ \approx & \text { homeomorfismo } \\ \mathscr{U}, \mathscr{V} & \text { coberturas abertas de um espaço topológico } \\ \bigoplus & \text { soma direta } \\ \coprod_{\vec{H}} & \text { união disjunta } \\ \breve{H}^{*} & \text { limite direto } \\ G & \text { cohomologia de Čech } \\ G x & \text { grupo topológico } \mid \text { compacto de Lie } \\ X / G & \text { órbita de } x \\ B G & \text { espaço de órbitas } \\ q_{X} & \text { espaços classificantes } \\ e & \text { aplicação classificante } \\ (E, p, B, G, G) & \text { elemento neutro } \\ X_{G} & \text { espaçrado principal } \\ f_{G} & \text { aplicação de Borel } \\ \smile & \text { produto cup } \\ R & \text { anel comutativo com identidade } \\ \mathbb{K} & \text { corpo } \\ \operatorname{Ind}^{G} & G \text {-índice de Fadell e Husseini de valor ideal } \\ \mid \text { Ind } & \text { k-índice de Fadell e Husseini de valor numérico } \\ \operatorname{ker}^{G} & \text { kernel do homomorfismo } f \\ \operatorname{deg} f & \text { grau de uma aplicação } \\ l, J, \ell & \text { inclusões }\end{array}$




\section{Introdução}

Dado um grupo topológico $G$ atuando sobre espaços topológicos $X$ e $Y$, uma aplicação $f: X \rightarrow Y$ é $G$-equivariante se $g \cdot f(x)=f(g \cdot x)$, para todo $x \in X$ e para todo $g \in G$. Um problema relevante em topologia é estabelecer a existência ou a não existência de aplicações $G$-equivariantes entre $G$-espaços e, quando possível, determinar o grau de tais aplicações.

Uma maneira eficaz de se abordar tais problemas é através da chamada teoria de $G$ índice. Possivelmente, C.T. Yang [54] foi o primeiro a definir um $\mathbb{Z}_{2}$-índice (co)homológico de forma explícita, para espaços com ação livre de $\mathbb{Z}_{2}$. Tal índice, o qual está conectado com o conceito de gênus, possui diversas aplicações em diferentes áreas da matemática. Para ações livres de grupos finitos arbitrários, tanto os índices homológicos, como os geométricos, foram primeiramente introduzidos por Shvarts [47],[48] e Conner-Floyd [15],[16]. Posteriormente, para o estudo dos espaços com ação de um grupo compacto de Lie, tornou-se necessária a definição de índices mais gerais, particularmente em aplicações a equações diferenciais não lineares, bifurcações e pontos críticos de aplicações $G$-equivariantes. Muitos autores têm dedicado seus estudos às definições e aplicações dos índices de tais $G$-espaços.

No sentido de se obter novas técnicas para a análise de aplicações $G$ - equivariantes, E. Fadell and S. Husseini, em [20] e [21], desenvolveram uma teoria de índice cohomológico denominada teoria de índice de valor ideal.

Anteriormente à publicação dos trabalhos de Fadell e Husseini, haviam sido considerados apenas índices numéricos, os quais associam a cada $G$-espaço, um número inteiro positivo (ou o símbolo $\infty$ ), com o objetivo de analisar a existência ou não de aplicações $G$-equivariantes. Tais resultados, muitas vezes conduzem a consequências importantes. Por exemplo, o teorema clássico de Borsuk-Ulam, o qual afirma que toda aplicação contínua de uma esfera $n$-dimensional no espaço euclidiano $\mathbb{R}^{n}$ colapsa pelo menos um par de pontos antípodas, é equivalente à afirmação de que não existe uma aplicação $\mathbb{Z}_{2}$-equivariante $S^{n} \rightarrow S^{n-1}$, onde $\mathbb{Z}_{2}$ atua livremente sobre as esferas, via uma involução $\mathbb{Z}_{2}$-equivariante.

No caso de grupos com anéis de cohomología suficientemente simples (por exemplo, 
álgebras polinomiais de uma variável), é conveniente utilizar índices numéricos. No entanto, invariantes numéricos são insuficientes no caso de grupos mais complexos, como por exemplo o toro e o p-toro. Neste contexto, Fadell e Husseini [20] introduziram o chamado Índice de valor ideal: associando a cada $G$-espaço $X$, um ideal $I n d^{G} X$ do anel de cohomología $\Lambda=H^{*}(B G)$, onde $H^{*}$ é uma apropriada teoria de cohomologia, com coeficientes em um corpo $\mathbb{K}$ e $B G$ é o espaço classificante do grupo $G$. O ideal Ind $^{G} X$ é considerado como sendo o kernel do homomorfismo entre as cohomologias equivariantes induzidas pela aplicação de $X$ em um espaço contendo apenas um ponto. É fácil mostrar que, se existe uma aplicação $G$-equivariante $\operatorname{de} X \operatorname{em} Y$, então $\operatorname{Ind}^{G} Y \subset$ Ind $^{G} X$.

A teoria de índice também está relacionada com a teoria de grau de uma aplicação $G$-equivariante. Em topologia algébrica, o grau é um invariante numérico que descreve uma aplicação contínua $f: M \rightarrow N$ entre duas variedades compactas, conexas, orientadas e de mesma dimensão. Intuitivamente, o grau representa o número de vezes que a variedade $M$ "envolve-se"na variedade $N$, sob a aplicação $f$. O grau de uma aplicação é sempre um número inteiro e foi definido pela primeira vez por Brouwer [9], que mostrou que o grau é um invariante por homotopia. Tal fato foi usado para provar o teorema do ponto fixo de Brouwer. Mais precisamente,

Definição Dada uma aplicação contínua $f: M \rightarrow N$ entre duas variedades orientadas compactas, conexas, sem bordo e de mesma dimensão $n$, com geradores $[M]$ e $[N]$ de $H^{n}(M ; \mathbb{Z})$ e $H^{n}(N ; \mathbb{Z})$, respectivamente, o grau de $f$ é definido como sendo o inteiro $\operatorname{deg} f$ tal que

$$
f^{*}([N])=(\operatorname{deg} f)[M] .
$$

O objetivo principal deste trabalho é apresentar um estudo detalhado da teoria de $G$ índice desenvolvida por Fadell e Husseini em [20] e [21], a qual é uma poderosa ferramenta com aplicações em diversas áreas da matemática. Aqui, nos restringiremos às aplicações no estudo do grau de funções $G$-equivariantes. Mais especificamente, apresentaremos uma descrição detalhada do seguinte resultado provado por Y.Hara em [24].

Teorema 4.4.4 [24, Theorem 1.1] Sejam $G$ um grupo compacto de Lie e $M, N G$-variedades compactas, conexas e sem bordo de dimensão finita $n$, que admitem uma $G$-ação livre. Então,

(1) Se $\operatorname{Ind}_{n-\operatorname{dim} G}^{G}\left(N, \mathbb{Z}_{2}\right)=\operatorname{Ind}_{n-\operatorname{dim} G}^{G}\left(M ; \mathbb{Z}_{2}\right) \neq H^{n-\operatorname{dim} G}\left(B G ; \mathbb{Z}_{2}\right)$, então para toda aplicação $G$-equivariante $f: M \rightarrow N$, temos que

$$
f^{*}: H^{n}\left(N ; \mathbb{Z}_{2}\right) \rightarrow H^{n}\left(M ; \mathbb{Z}_{2}\right)
$$

é um isomorfismo. Além disso, se $M$ e $N$ são orientáveis, então para toda aplicação $G$ equivariante $f: M \rightarrow N$, o grau de $f$ é ímpar.

(2) Se $\operatorname{Ind}_{n-\operatorname{dim} G}^{G}\left(N, \mathbb{Z}_{2}\right) \neq \operatorname{Ind}_{n-\operatorname{dim} G}^{G}\left(M ; \mathbb{Z}_{2}\right)=H^{n-\operatorname{dim} G}\left(B G ; \mathbb{Z}_{2}\right)$, então para toda aplicação $G$-equivariante $f: M \rightarrow N$, temos que

$$
f^{*}: H^{n}\left(N ; \mathbb{Z}_{2}\right) \rightarrow H^{n}\left(M ; \mathbb{Z}_{2}\right)
$$

é o homorfismo trivial. Além disso, se $M$ e $N$ são orientáveis, então para toda aplicação $G$-equivariante $f: M \rightarrow N$, o grau de $f$ é par. 
J.Jaworowski em [32] provou um resultado similar ao teorema de Hara. Em suas demonstrações, ambos utilizaram como ferramenta a cohomologia especial de Smith para $\mathbb{Z}_{p}$-espaços livres.

Gonçalves, Pergher, Jaworowski e Volovikov em [18] mostraram a existência de um homomorfismo transfer $\tau_{X}: H^{i}(X ; R) \rightarrow H^{i}(X / G ; R)$ para $G$-espaços livres, com $G$ um grupo finito. Tal homomorfismo possui a propriedade de ser sobrejetor, quando o $G$ espaço for um CW- complexo de dimensão finita. Usando este resultado, apresentamos uma demonstração alternativa do teorema provado por Hara[24] e Jaworowski [32], a qual simplifica consideravelmente a prova apresentada por eles. 


\section{Cohomologia de Čech}

Este capítulo introduz a Teoria de cohomologia de Čech, a qual será usada, no Capítulo 3, para definir a cohomologia $G$-equivariante de Borel. A idéia básica dessa construção é associar a um par de espaços topológicos $(X, A)$, pares simpliciais $\left(K_{\mathscr{U}}, L_{\mathscr{U}}\right)$, onde $\mathscr{U}$ é uma cobertura aberta de $X$. Os $R$-módulos de cohomologia de Čech do par $(X, A)$ são definidos então, como sendo o limite direto dos $R$-módulos de cohomologia simplicial relativos $H^{q}\left(K_{\mathscr{U}}, L_{\mathscr{U}} ; R\right)$, onde $R$ é um anel comutativo com identidade. Para a construção da cohomologia de Čech apresentamos inicialmente, na Seção 1.1, uma descrição do conceito de limite direto de $R$-módulos. Na Subseção 1.1.2, é introduzido o conceito de limite direto de espaços topológicos, que será usado na construção de Milnor, descrita no Capítulo 2. Na Seção 1.2 apresentamos os $R$-módulos de cohomologia simplicial relativos, a partir dos quais a cohomologia de Čech é definida, na Seção 1.3. As referências básicas para este capítulo são o artigo Čech cohomology theory and the axioms de C.H.Dowker [14] e [53].

\subsection{Limite direto}

DEFINIÇ̃̃o 1.1.1. Seja $(\Lambda, \leq)$ um conjunto pré-ordenado. ${ }^{1}$ Dizemos que $(\Lambda, \leq)$ é um conjunto dirigido se, para cada par de pontos $\alpha_{1}$ e $\alpha_{2} \in \Lambda$, existe $\alpha_{3} \in \Lambda$ tal que $\alpha_{1} \leq \alpha_{3}$ e $\alpha_{2} \leq \alpha_{3}$.

OBSERVAÇão 1.1.2. Alguns autores consideram na Definição 1.1.1 a relação $\leq$ como sendo uma relação de ordem parcial ${ }^{2}$.

DEFINIÇão 1.1.3. Seja $(\Lambda, \leq)$ um conjunto dirigido. Um sistema direto

$$
\left\{X_{\alpha}, f^{\beta \alpha}, \Lambda\right\}
$$

\footnotetext{
${ }^{1}$ Uma relação binária $\leq$ sobre um conjunto $\Lambda$ é chamada uma pré-ordem se $\leq$ for reflexiva e transitiva. Um conjunto $\Lambda$ munido de uma pré-ordem é chamado um conjunto pré-ordenado e será denotado por $(\Lambda, \leq)$.

${ }^{2}$ Seja $(\Lambda, \leq)$ um conjunto pré-ordenado. Se $\leq$ satisfaz a propriedade adicional: $a \leq b$ e $b \leq a \Rightarrow a=b$ (antisimétrica), então $\Lambda$ é chamado um conjunto parcialmente ordenado.
} 
em uma categoria $\mathbf{C}$, consiste de uma família de objetos $\left\{X_{\alpha}\right\}_{\alpha \in \Lambda}$ em $\mathbf{C}$ indexados no conjunto dirigido $\Lambda$ e uma família de morfismos de $\mathbf{C}$

$$
\left\{f^{\beta \alpha}: X_{\beta} \rightarrow X_{\alpha} \text {, para cada } \alpha \leq \beta\right\},
$$

satisfazendo as seguintes condições

(i) $f^{\alpha \alpha}=\operatorname{Id}_{X_{\alpha}}: X_{\alpha} \rightarrow X_{\alpha}$.

(ii) $f^{\gamma \alpha}=f^{\gamma \beta} \circ f^{\beta \alpha}: X_{\alpha} \rightarrow X_{\gamma}$, se $\alpha \leq \beta \leq \gamma$.

\subsubsection{Limite direto de $R$-módulos}

Sejam $R$ um anel comutativo com identidade $1_{R} \mathrm{e}$

$$
\left\{M_{\alpha}, f^{\beta \alpha}, \Lambda\right\}
$$

um sistema direto de $R$-módulos, ou seja, $\left\{M_{\alpha}\right\}_{\alpha \in \Lambda}$ é um familia de $R$-módulos indexada em um conjunto dirigido $\Lambda \mathrm{e}$

$$
\left\{f^{\beta \alpha}: M_{\alpha} \rightarrow M_{\beta} ; \text { para cada } \alpha \leq \beta\right\}
$$

é uma familia de $R$-homomorfismos satisfazendo as condições (i) e (ii) da Definição 1.1.3. Consideremos a soma direta dos $R$-módulos $M_{\alpha}: \bigoplus_{\alpha \in \Lambda} M_{\alpha}$ e observemos que seus elementos podem ser escritos de maneira única como uma soma finita $m_{\alpha_{1}}+m_{\alpha_{2}}+\cdots+$ $m_{\alpha_{n}}$, onde cada $m_{\alpha_{i}} \in M_{\alpha_{i}}$ e $i=1, \ldots, n$ (Vide [28, Pg.22]). Seja $S$ o seguinte subconjunto $\operatorname{de} \bigoplus_{\alpha \in \Lambda} M_{\alpha}$,

$$
S=\left\{m_{\alpha}-f^{\beta \alpha}\left(m_{\alpha}\right) ; \alpha, \beta \in \Lambda, \operatorname{com} \beta \leq \alpha\right\},
$$

onde $f^{\beta \alpha}: M_{\alpha} \rightarrow M_{\beta}$, para cada $\alpha \leq \beta$ e consideremos o submódulo $\langle S\rangle$ de $\bigoplus_{\alpha \in \Lambda} M_{\alpha}$ gerado por $S$.

DEFINIÇão 1.1.4. Definimos o limite direto do sistema direto $\left\{M_{\alpha}, f^{\beta \alpha}, \Lambda\right\}$ de $R$-módulos como sendo o $R$-módulo quociente:

$$
\lim _{\alpha \in \Lambda} M_{\alpha}=\frac{\bigoplus_{\alpha \in \Lambda} M_{\alpha}}{\langle S\rangle} .
$$

OBSERVAÇ̃̃o 1.1.5. Seja $\left\{M_{\alpha}, f^{\beta \alpha}, \Lambda\right\}$ um sistema direto de $R$-módulos. Se

$$
m \in \underset{\alpha \in \Lambda}{\lim } M_{\alpha},
$$

então $m$ possui um representante em $\bigoplus_{\alpha \in \Lambda} M_{\alpha}$, o qual é exatamente um elemento $m_{\alpha} \in$ $M_{\alpha}$, para algum $\alpha \in \Lambda$, ou seja, $m=m_{\alpha}+\langle S\rangle$, para algum $\alpha \in \Lambda$ (Vide [53, Pg.242]).

obSERVAÇão 1.1.6. Observemos que se $m_{\alpha} \in \bigoplus_{\alpha \in \Lambda} M_{\alpha}$ é tal que $m_{\alpha} \in\langle S\rangle$, então existe $\beta \in \Lambda, \operatorname{com} \alpha \leq \beta$ tal que $f^{\beta \alpha}\left(m_{\alpha}\right)=0$ (Vide [53, Pg.242]). 
DEFINIÇão 1.1.7. Sejam $\left\{M_{\alpha}, f^{\beta \alpha}, \Lambda\right\}$ e $\left\{N_{\alpha^{\prime}}, g^{\beta^{\prime} \alpha^{\prime}}, \Lambda^{\prime}\right\}$ dois sistemas diretos de $R$-módulos, indexados pelos conjuntos dirigidos $\Lambda$ e $\Lambda^{\prime}$, respectivamente. Seja $\phi: \Lambda \rightarrow \Lambda^{\prime}$ uma aplicação preservando a relação de ordem. Um sistema direto de $R$-homomorfismos é uma família de $R$-homomorfismos $\left\{f_{\alpha}: M_{\alpha} \rightarrow N_{\phi(\alpha)} ; \alpha \in \Lambda\right\}$, tal que o seguinte diagrama é comutativo

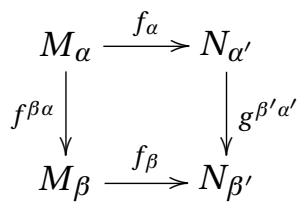

onde $\alpha^{\prime}=\phi(\alpha) \leq \phi(\beta)=\beta^{\prime}$, para todo $\alpha \leq \beta$.

OBSERVAÇÃo 1.1.8. A familia $\left\{f_{\alpha}\right\}_{\alpha \in \Lambda}$ induz um $R$-homomorfismo

$$
\oplus f_{\alpha}: \oplus_{\alpha \in \Lambda} M_{\alpha} \rightarrow \oplus_{\alpha^{\prime} \in \Lambda^{\prime}} N_{\alpha^{\prime}}
$$

definido por $\left(\oplus f_{\alpha}\right)\left(m_{\alpha_{1}}+\cdots+m_{\alpha_{n}}\right)=f_{\alpha_{1}}\left(m_{\alpha_{1}}\right)+\cdots+f_{\alpha_{n}}\left(m_{\alpha_{n}}\right)$.

Observemos que o elemento $m_{\alpha}-f^{\beta \alpha}\left(m_{\alpha}\right)$ é levado por esta aplicação no elemento $f_{\alpha}\left(m_{\alpha}\right)-f_{\beta}\left(f^{\beta \alpha}\left(m_{\alpha}\right)\right)=f_{\alpha}\left(m_{\alpha}\right)-g^{\beta^{\prime} \alpha^{\prime}}\left(f_{\alpha}\left(m_{\alpha}\right)\right)$, onde a última igualdade segue da comutatividade do diagrama 1.2.

Assim, os elementos de $\langle S\rangle$ em $\bigoplus_{\alpha \in \Lambda} M_{\alpha}$ são levados por $\oplus f_{\alpha}$ em elementos do submódulo correspondente $\left\langle S^{\prime}\right\rangle$ de $\bigoplus_{\alpha^{\prime} \in \Lambda^{\prime}} N_{\alpha^{\prime}}$ e, portanto, $\oplus f_{\alpha}$ induz um homomorfismo entre os $R$-módulos quocientes

$$
\begin{aligned}
& f=\lim _{\alpha \in \Lambda} f_{\alpha}: \underset{\alpha \in \Lambda}{\lim _{\alpha \in \Lambda}} M_{\alpha} \rightarrow \underset{\alpha^{\prime} \in \Lambda^{\prime}}{\lim _{\alpha^{\prime}}} N_{\alpha^{\prime}} \text {, onde } \\
& \left(\underset{\alpha \in \Lambda}{\lim _{\alpha \in \Lambda} f_{\alpha}}\right)\left(m_{\alpha}+\langle S\rangle\right)=f_{\alpha}\left(m_{\alpha}\right)+\left\langle S^{\prime}\right\rangle .
\end{aligned}
$$

DEFINIÇÃO 1.1.9. O homomorfismo $f=\lim _{\rightarrow} f_{\alpha}$ definido em 1.3 é chamado o limite direto dos $R$-homomorfismos $f_{\alpha}$.

OBSERVAÇÃO 1.1.10. Observemos da Definição 1.1.7 que o limite direto dos $R$-homomorfismos $f_{\alpha}$ é determinado também pela aplicação $\phi: \Lambda \rightarrow \Lambda^{\prime}$, a qual preserva a relação de ordem entre os conjuntos dirigidos $\Lambda$ e $\Lambda^{\prime}$.

OBSERVAÇÃo 1.1.11. Sejam $\left\{M_{\alpha}, f^{\alpha \beta}, \Lambda\right\},\left\{N_{\alpha^{\prime}}, g^{\alpha^{\prime} \beta^{\prime}}, \Lambda^{\prime}\right\}$ e $\left\{P_{\alpha^{\prime \prime}}, h^{\alpha^{\prime \prime} \beta^{\prime \prime}}, \Lambda^{\prime \prime}\right\}$ sistemas diretos, $\phi: \Lambda \rightarrow \Lambda^{\prime}$ e $\psi: \Lambda^{\prime} \rightarrow \Lambda^{\prime \prime}$ aplicações preservando as respectivas relações de ordem. Dados os sistemas diretos de $R$-homomorfismos

$$
\left\{f_{\alpha}: M_{\alpha} \rightarrow N_{\phi(\alpha)} ; \alpha \in \Lambda\right\} \text { e }\left\{g_{\alpha^{\prime}}: N_{\alpha^{\prime}} \rightarrow P_{\psi\left(\alpha^{\prime}\right)} ; \alpha^{\prime} \in \Lambda^{\prime}\right\}
$$


onde $\phi(\alpha)=\alpha^{\prime}$ e $\psi\left(\alpha^{\prime}\right)=\alpha^{\prime \prime}$, temos que a composição $\psi \circ \phi: \Lambda \rightarrow \Lambda^{\prime \prime}$ é uma aplicação que preserva ordem e, desde que pela Definição 1.1.7, cada quadrado no seguinte diagrama é comutativo,

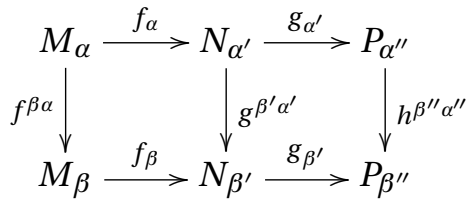

segue que o diagrama a seguir também é comutativo

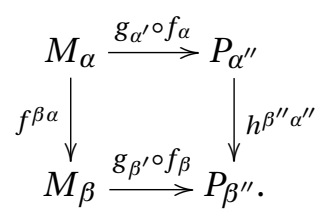

Portanto, a família de $R$-homomorfismos $\left\{g_{\alpha^{\prime}} \circ f_{\alpha}: M_{\alpha} \rightarrow P_{\alpha^{\prime \prime}}\right\}$ é um sistema direto de

$R$-homomorfismos. Além disso, se $m_{\alpha}+\langle S\rangle \in \underset{\alpha \in \Lambda}{\lim } M_{\alpha}$, temos

$$
\begin{aligned}
& \left(\underset{\lim ^{\prime} \in \Lambda^{\prime}}{ } g_{\alpha^{\prime}}\right)\left(\lim _{\alpha \in \Lambda} f_{\alpha}\right)\left(m_{\alpha}+\langle S\rangle\right)=\lim _{\substack{\alpha^{\prime} \in \Lambda^{\prime}}} g_{\alpha^{\prime}}\left(f_{\alpha}\left(m_{\alpha}\right)+\left\langle S^{\prime}\right\rangle\right) \\
& =g_{\alpha^{\prime}} \circ f_{\alpha}\left(m_{\alpha}\right)+\left\langle S^{\prime \prime}\right\rangle \\
& =\left(\lim _{\alpha \in \Lambda} g_{\alpha^{\prime}} \circ f_{\alpha}\right)\left(m_{\alpha}+\langle S\rangle\right) .
\end{aligned}
$$

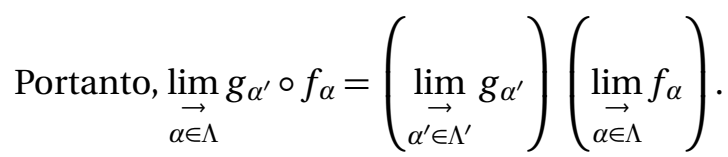

DEFINIÇÃo 1.1.12. Seja $\Lambda$ um conjunto dirigido. Um subconjunto $\Lambda_{0}$ de $\Lambda$ é chamado cofinal em $\Lambda$ se, para cada $\alpha \in \Lambda$, existe $\beta \in \Lambda_{0} \operatorname{com} \alpha \leq \beta$.

OBSERVAÇÃo 1.1.13. Seja $\Lambda$ um conjunto dirigido. Se $\Lambda_{0} \subset \Lambda$ for cofinal em $\Lambda$, então $\Lambda_{0}$ é um conjunto dirigido pela relação de ordem de $\Lambda$. De fato, dados $\alpha_{1} \alpha_{2} \in \Lambda_{0} \subset \Lambda$, desde que $\Lambda$ é um conjunto dirigido, existe $\alpha_{3} \in \Lambda$ tal que $\alpha_{1} \leq \alpha_{3}$ e $\alpha_{2} \leq \alpha_{3}$. Além disso, como $\Lambda_{0}$ é cofinal em $\Lambda$, para este $\alpha_{3} \in \Lambda$, existe $\beta \in \Lambda_{0}$ tal que $\alpha_{1} \leq \alpha_{3} \leq \beta$ e $\alpha_{2} \leq \alpha_{3} \leq \beta$. Portanto, $\Lambda_{0}$ é um conjunto dirigido.

PROPOSIÇÃo 1.1.14. Sejam $\left\{M_{\alpha}, f^{\beta \alpha}, \Lambda\right\}$ um sistema direto de R-módulos indexado pelo conjunto dirigido $\Lambda$ e $\Lambda_{0} \subset \Lambda$ cofinal em $\Lambda$. Então,

$$
\lim _{\alpha \in \Lambda_{0}} M_{\alpha} \cong \underset{\alpha \in \Lambda}{\lim } M_{\alpha}
$$


Demonstração: Dado o sistema direto de $R$-módulos $\left\{M_{\alpha}, f^{\beta \alpha}, \Lambda\right\}$, indexado pelo conjunto dirigido $\Lambda$, desde que $\Lambda_{0}$ é cofinal em $\Lambda$, temos da Observação 1.1.13 que $\left\{M_{\alpha}, f^{\alpha \beta}, \Lambda_{0}\right\}$ é um sistema direto de $R$-módulos. Consideremos a inclusão $\phi: \Lambda_{0} \hookrightarrow \Lambda$, a qual é uma aplicação que preserva a relação de ordem. Assim, temos que a família de $R$-homomorfismos

$$
\left\{\mathrm{Id}=f_{\alpha}: M_{\alpha} \rightarrow M_{\phi(\alpha)}: \alpha \in \Lambda_{0}\right\}
$$

é um sistema direto de $R$-homomorfismos. Seja

$$
f=\lim _{\alpha \in \Lambda_{0}} f_{\alpha}: \lim _{\alpha \in \Lambda_{0}} M_{\alpha} \rightarrow \lim _{\alpha \in \Lambda} M_{\alpha}
$$

o limite direto dos $R$-homomorfismos $f_{\alpha}$ e mostremos que $f$ é um isomorfismo. Dada uma classe lateral

$$
m_{\alpha}+\langle S\rangle \in \underset{\alpha \in \Lambda}{\lim } M_{\alpha}
$$

para algum $\alpha \in \Lambda$, segue da cofinalidade de $\Lambda_{0}$ que existe $\beta \in \Lambda_{0}$ tal que $\alpha \leq \beta$. Seja $f^{\beta \alpha}\left(m_{\alpha}\right)=m_{\beta}$, então $m_{\beta}+\left\langle S_{0}\right\rangle \in \underset{\alpha \in \Lambda_{0}}{\lim _{\alpha}} M_{\alpha}$ e

$$
\begin{aligned}
\underset{\alpha \in \Lambda_{0}}{\left(\lim _{\alpha} f_{\alpha}\right)\left(m_{\beta}+\left\langle S_{0}\right\rangle\right)} & =f_{\beta}\left(m_{\beta}\right)+\langle S\rangle \\
& =m_{\beta}+\langle S\rangle \\
& =m_{\beta}+\left[m_{\alpha}-f^{\beta \alpha}\left(m_{\alpha}\right)\right]+\langle S\rangle \\
& =m_{\beta}+\left[m_{\alpha}-m_{\beta}\right]+\langle S\rangle \\
& =m_{\alpha}+\langle S\rangle
\end{aligned}
$$

desde que, por 1.1, $m_{\alpha}-f^{\beta \alpha}\left(m_{\alpha}\right) \in\langle S\rangle$. Portanto, $\underset{\alpha \in \Lambda_{0}}{\lim _{\alpha}} f_{\alpha}$ é sobrejetora.

Seja $m_{\alpha}+\left\langle S_{0}\right\rangle \in \lim _{\alpha \in \Lambda_{0}} M_{\alpha}$ tal que $m_{\alpha}+\left\langle S_{0}\right\rangle$ pertence ao núcleo de $f$. Assim,

$$
\left(\underset{\alpha \in \Lambda_{0}}{\left(\lim _{\alpha}\right.} f_{\alpha}\right)\left(m_{\alpha}+\left\langle S_{0}\right\rangle\right)=f_{\alpha}\left(m_{\alpha}\right)+\langle S\rangle=m_{\alpha}+\langle S\rangle=\langle S\rangle
$$

o que implica que $m_{\alpha} \in\langle S\rangle$. Segue da Observação 1.1.6 que $f^{\beta \alpha}\left(m_{\alpha}\right)=0$, para algum $\beta \in \Lambda$, com $\alpha \leq \beta$. Pela cofinalidade de $\Lambda_{0}$, para este $\beta \in \Lambda$, existe $\gamma \in \Lambda_{0}$ tal que $\beta \leq \gamma$. Então,

$$
f^{\gamma \alpha}\left(m_{\alpha}\right)=f^{\gamma \beta} \circ f^{\beta \alpha}\left(m_{\alpha}\right)=0, \operatorname{com} \alpha \leq \beta \leq \gamma
$$

Logo, $m_{\alpha}=m_{\alpha}-f^{\gamma \alpha}\left(m_{\alpha}\right)$, onde $\alpha, \gamma \in \Lambda_{0}$ são tais que $\alpha \leq \gamma$, o que implica de 1.1, que $m_{\alpha} \in\left\langle S_{0}\right\rangle$, ou seja, $m_{\alpha}+\left\langle S_{0}\right\rangle=\left\langle S_{0}\right\rangle$. Portanto, $f$ é injetora. 


\subsubsection{Limite direto de espaços topológicos}

DEFINIÇÃo 1.1.15. Sejam $\Lambda$ um conjunto dirigido e $\left\{X_{\alpha} ; \alpha \in \Lambda\right\}$ uma família de espaços topológicos indexada por $\Lambda$. Dada uma família de funções contínuas

$$
\left\{f^{\beta \alpha}: X_{\alpha} \rightarrow X_{\beta} \text {, para cada par de índices } \alpha, \beta \in \Lambda \text { tais que } \alpha \leq \beta\right\}
$$

satisfazendo as condições (i) e (ii) da Definição 1.1.3, então $\left\{X_{\alpha}, f^{\beta \alpha}, \Lambda\right\}$ é chamado um sistema direto de espaços topológicos .

DEFINIÇão 1.1.16. Seja $\left\{X_{\alpha} ; \alpha \in \Lambda\right\}$ uma família de espaços topológicos indexada em um conjunto de índices arbitrário $\Lambda$. Para cada $\alpha \in \Lambda$, seja

$$
X_{\alpha}^{*}=\left\{(x, \alpha) ; x \in X_{\alpha}\right\}=X_{\alpha} \times\{\alpha\},
$$

o qual é homeomorfo a $X_{\alpha}$. Observemos que $X_{\alpha}^{*} \cap X_{\beta}^{*}=\emptyset$, se $\alpha \neq \beta$, ou seja, o conjunto $\left\{X_{\alpha}^{*} ; \alpha \in \Lambda\right\}$ é uma família de espaços topológicos dois a dois disjuntos. Definimos uma topologia sobre

$$
X=\bigcup_{\alpha \in \Lambda} X_{\alpha}^{*}
$$

como segue: $U \subset X$ é aberto em $X$ se, e somente se, $U \cap X_{\alpha}^{*}$ for aberto em $X_{\alpha}^{*}$, para cada $\alpha \in \Lambda$. $X$ munido desta topologia é chamado a união disjunta, (ou união livre ou ainda soma topológica ) dos espaços $X_{\alpha}$ e será denotado por

$$
\sum_{\alpha \in \Lambda} X_{\alpha}
$$

DEFINIÇÃo 1.1.17. Sejam $\left\{X_{\alpha}, f^{\beta \alpha}, \Lambda\right\}$ um sistema direto de espaços topológicos. Definimos sobre $\sum_{\alpha \in \Lambda} X_{\alpha}$ a seguinte relação de equivalência: dados $x_{\alpha} \in X_{\alpha}$ e $x_{\beta} \in X_{\beta}$, então

$$
x_{\alpha} \sim x_{\beta} \Leftrightarrow \exists \gamma \in \Lambda, \text { com } \alpha \leq \gamma \text { e } \beta \leq \gamma \text { tal que } f^{\gamma \alpha}\left(x_{\alpha}\right)=f^{\gamma \beta}\left(x_{\beta}\right) .
$$

O limite direto do sistema direto $\left\{X_{\alpha}, f^{\beta \alpha}, \Lambda\right\}$, o qual será denotado por

$$
\lim _{\alpha \in \Lambda} X_{\alpha} \text { ou } X^{\infty}
$$

é definido como sendo o espaço quociente

$$
\lim _{\vec{\alpha} \in \Lambda} X_{\alpha}=\frac{\sum_{\alpha \in \Lambda} X_{\alpha}}{\sim}=\left\{\left[x_{\alpha}\right]: x_{\alpha} \in X_{\alpha}\right\}
$$

PRoposiçÃo 1.1.18. Seja $\left\{X_{\alpha}, f^{\beta \alpha}, \Lambda\right\}$ um sistema direto de espaços topológicos. Suponhamos que para cada $\alpha, \beta \in \Lambda$ tais que $\alpha \leq \beta$, as aplicações contínuas $f^{\beta \alpha}: X_{\alpha} \hookrightarrow X_{\beta}$ sejam inclusões. Então,

$$
\lim _{\alpha \in \Lambda} X_{\alpha} \stackrel{\text { def }}{=} \frac{\sum_{\alpha \in \Lambda} X_{\alpha}}{\sim}=\sum_{\alpha \in \Lambda} X_{\alpha}
$$


Demonstração: Sejam $x_{\alpha}, x_{\beta} \in \sum_{\alpha \in \Lambda} X_{\alpha}$ tais que $x_{\alpha} \sim x_{\beta}$. Então, existe $\gamma \in \Lambda$ com $\alpha \leq \gamma$ e $\beta \leq \gamma$ tal que $x_{\alpha}=f^{\gamma \alpha}\left(x_{\alpha}\right)=f^{\gamma \beta}\left(x_{\beta}\right)=x_{\beta}$, desde que as aplicações $f^{\gamma \alpha}: X_{\alpha} \hookrightarrow X_{\gamma} \mathrm{e}$ $f^{\gamma \beta}: X_{\beta} \hookrightarrow X_{\gamma}$ são inclusões. Portanto, $\left[x_{\alpha}\right]=\left\{x_{\alpha}\right\}$, para todo $x_{\alpha} \in \sum_{\alpha \in \Lambda} X_{\alpha}$. Segue o resultado.

OBSERVAÇÃo 1.1.19. Seja $\left\{X_{\alpha}, f^{\beta \alpha}, \Lambda\right\}$ um sistema direto de espaços topológicos nas mesmas hipóteses da Proposição 1.1.18. Se cada $X_{\alpha}$ for um espaço compacto Hausdorff, então,

$$
\lim _{\alpha \in \Lambda} X_{\alpha}=\sum_{\alpha \in \Lambda} X_{\alpha}
$$

é paracompacto ${ }^{3}$, desde que que a união disjunta de espaços pacompactos é um espaço paracompacto (Vide [27, 9 DUP, Pg.495]).

EXEMPlo 1.1.20. Dado o sistema direto $\left\{S^{n}, \imath, \mathbb{Z}^{+}\right\}$, onde $\imath: S^{n} \hookrightarrow S^{m}$, é a inclusão, para $n \leq m$, segue da Proposição 1.1.18 que

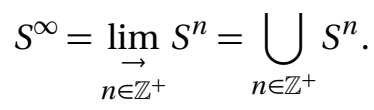

\subsection{Cohomologia simplicial}

Esta seção introduz a construção dos $R$-módulos de cohomologia simplicial relativos, os quais satisfazem os Axiomas de Eilenberg-Steenrod. Para essa construção, usamos como referências [43] e [49].

\subsubsection{A categoria dos pares simpliciais}

Inicialmente, apresentamos as noções básicas sobre a categoria dos pares simpliciais e das aplicações de pares simpliciais, necessárias para o desenvolvimento deste trabalho.

DEFINIÇÃO 1.2.1. Um complexo simplicial abstrato $K$ é um par $(V, S)$ onde $V$ é um conjunto e $S$ é uma coleção de subconjuntos finitos e não vazios de $V$ satisfazendo as seguintes condições:

(i) Se $v \in V$, então $\{v\} \in S$.

(ii) Se $\tau \subset \sigma \in S$ e se $\tau \neq \emptyset$, então $\tau \in S$.

Os elementos de $v \in V$ são chamados vértices e os elementos $\sigma \in S$ são chamados simplexos. Com essa terminologia, a condição $(i)$ afirma que qualquer conjunto consistindo de exatamente um vértice é um simplexo e a condição $(i i)$ diz que qualquer subconjunto não vazio de um simplexo é um simplexo. Um $q$-simplexo é um elemento de $S \operatorname{com} q+1$ vértices. Se $\sigma \in S$ for um $q$-simplexo, dizemos que $\operatorname{dim}(\sigma)=q$. Se $\tau \subset \sigma, \tau$ é chamado uma face de $\sigma$, se $\tau \neq \sigma$, $\tau$ é chamada uma face própria de $\sigma$ e se $\tau$ for um $p$-simplexo, ele é chamado uma $p$-face de $\sigma$. Se $\sigma$ for um $q$-simplexo, então $\sigma$ é a única $q$-face de $\sigma$ e $\tau \subset \sigma$ é uma face própria de $\sigma$ se, e somente se, $\operatorname{dim} \tau<q$.

\footnotetext{
${ }^{3}$ Vide Definição A.3.5
} 
OBSERVAÇÃo 1.2.2. Seja $K$ um complexo simplicial abstrato. Observemos que qualquer simplexo $\sigma \in K$ possui um número finito de faces e, desde que, toda face de uma face de $\sigma$ é ela própria uma face de $\sigma$, os simplexos de $K$ são parcialmente ordenados pela relação: $\tau \subset \sigma$, se $\tau$ for uma face de $\sigma$. Segue da condição (i) da Definição 1.2.1 que existe uma correspondência bijetora entre os 0 -simplexos de $K$ e os vértices de $K$. E, da condição (ii), temos que qualquer simplexo é determinado por suas 0 -faces. Portanto, $K$ pode ser considerado como sendo igual ao conjunto de seus simplexos e identificamos um vértice de $K$ com o 0 -simplexo correspondente a ele.

EXEMPLO 1.2.3. Existe um complexo simplicial, cujo conjunto de vértices é $V=\mathbb{Z}$ e cujo conjunto de simplexos é

$$
S=\{\{n\} ; n \in \mathbb{Z}\} \cup\{\{n, n+1\} ; n \in \mathbb{Z}\} .
$$

Nesse caso, para cada $n \in \mathbb{Z}, K$ contém os 0 -simplexos $\{n\}$ e os 1-simplexos $\{n, n+1\}$.

DEFINIÇÃo 1.2.4. Um subcomplexo $L$ de um complexo simplicial $K$, o qual será denotado por $L \subset K$, é um subconjunto de $K$ (ou seja, $\sigma \in L \Rightarrow \sigma \in K$ ) que é ele próprio um complexo simplicial.

EXEMPLO 1.2.5. Dado um complexo simplicial $K$, o seu $q$-dimensional esqueleto $K^{(q)}$ é definido como sendo o subcomplexo consistindo de todos os $p$-simplexos de $K$, para $p \leq q$.

OBSERVAÇão 1.2.6. Um subconjunto $L$ de $K$ é um subcomplexo de $K$ se, e somente se, dado qualquer simplexo $\tau$ em $K$ que é uma face de um simplexo de $L$, ou seja, $\tau \subset \sigma$, para algum $\sigma \in L$, então $\tau$ é um simplexo de $L$.

DEFINIÇão 1.2.7. Se $K$ for um complexo simplicial, sua dimensão, denotada por $\operatorname{dim} K$, é definida como sendo -1 , se $K$ for vazio e é igual a $n$, se $K$ contém um $n$-simplexo, mas não contém nenhum $(n+1)$-simplexo. Se $K$ contém $n$-simplexos, para todo $n \geq 0$, dizemos que $\operatorname{dim} K$ é infinita e, nesse caso, $K$ é chamado um complexo simplicial infinitodimensional. Assim, $\operatorname{dim} K=\sup \{\operatorname{dim} \sigma ; \sigma \in K\}$. Dizemos que $K$ é finito se ele contém apenas um número finito de simplexos.

OBSERVAÇÃo 1.2.8. Se $K$ for um complexo simplicial finito, então temos que $\operatorname{dim} K<\infty$. Entretanto, se $\operatorname{dim} K<\infty, K$ não é necessariamente finito. O Exemplo 1.2.3, mostra um complexo simplicial infinito-dimensional $K$ tal que $\operatorname{dim} K=1$.

DEFINIÇÃo 1.2.9. Dados dois complexos simpliciais abstratos $K_{1}$ e $K_{2}$, uma aplicação simplicial $\varphi: K_{1} \rightarrow K_{2}$ é uma função $\varphi$ do conjunto de vértices de $K_{1}$ no conjunto de vértices de $K_{2}$ com a seguinte propriedade: para qualquer simplexo $\sigma=\left\{v_{0}, \ldots, v_{q}\right\} \in K_{1}$, sua imagem $\varphi(\sigma)=\left\{\varphi\left(v_{0}\right), \ldots, \varphi\left(v_{q}\right)\right\}$ é um simplexo de $K_{2}$. Especificamente, uma aplicação simplicial $\varphi: K_{1} \rightarrow K_{2}$ é da forma $\varphi\left(\left\{v_{0}, \ldots, v_{q}\right\}\right)=\left\{\varphi_{0}\left(\nu_{0}\right), \ldots, \varphi_{0}\left(v_{q}\right)\right\}$, para alguma aplicação $\varphi_{0}: K_{1}^{(0)} \rightarrow K_{2}^{(0)}$, chamada aplicação vértice de $\varphi$, onde $K_{1}^{(0)}$ e $K_{2}^{(0)}$ denotam os 0 -esqueletos de $K_{1}$ e $K_{2}$, respectivamente. 
OBSERVAÇÃo 1.2.10. Para qualquer complexo simplicial abstrato $K$, existe uma aplicação simplicial identidade $I d_{K}: K \rightarrow K$, a qual corresponde a uma aplicação vértice identidade. Se $L \subset K$, existe uma inclusão natural $\imath: L \hookrightarrow K$, a qual é um aplicação simplicial. Dadas aplicações simpliciais

$$
K_{1} \stackrel{\varphi}{\rightarrow} K_{2} \stackrel{\psi}{\rightarrow} K_{3}
$$

então a composição $\psi \circ \varphi: K_{1} \rightarrow K_{3}$ é uma aplicação simplicial, a qual corresponde a uma aplicação vértice composição.

DEFINIÇÃO 1.2.11. Um par simplicial é um par $(K, L)$, onde $K$ é um complexo simplicial abstrato e $L$ é um subcomplexo de $K$. Uma aplicação de pares simpliciais $\varphi:\left(K_{1}, L_{1}\right) \rightarrow$ $\left(K_{2}, L_{2}\right)$ é uma aplicação simplicial $\varphi: K_{1} \rightarrow K_{2}$ tal que $\varphi\left(L_{1}\right) \subset L_{2}$, ou seja, $\varphi$ leva cada simplexo de $L_{1}$ em um simplexo de $L_{2}$.

Existe uma categoria, cujos objetos são pares simpliciais $(K, L)$ e cujos morfismos são aplicações de pares simpliciais $\varphi:\left(K_{1}, L_{1}\right) \rightarrow\left(K_{2}, L_{2}\right)$.

\subsubsection{Os $R$-módulos de cohomologia simplicial relativos}

Nesta seção, apresentamos um breve resumo sobre a construção dos $R$-módulos de cohomologia simplicial relativos, onde $R$ é um anel comutativo com identidade.

DEFINIÇÃo 1.2.12. Sejam $K$ um complexo simplicial abstrato. Um $q$-simplexo orientado de $K$ é um $q$-simplexo $\sigma \in K$ junto com uma classe de equivalência, determinada pelas ordenações dos vértices de $\sigma$. Dadas duas ordenações $v_{i_{0}}<\ldots<v_{i_{q}}$ e $v_{j_{0}}<\ldots<v_{j_{q}}$ dos vértices de $\sigma$, existe uma permutação $P$ do conjunto $\{0, \ldots, q\}$ tal que $P\left(i_{k}\right)=j_{k}$, para $k=$ $0, \ldots, q$. Definimos uma relação de equivalência sobre o conjunto de todas as ordenações dos vértices de $\sigma$ da seguinte forma: duas ordenações são equivalentes se, e somente se, elas diferem por uma permutação par dos vértices de $\sigma$. Uma escolha de uma classe de equivalência de ordenações dos vértices de $\sigma$ é chamada uma orientação para $\sigma$. Se $v_{0}, \ldots, v_{q}$ são vértices de $\sigma$, então $\left[v_{0}, v_{1}, \ldots, v_{q}\right]$ denotará o $q$-simplexo orientado de $K$ consistindo do simplexo $\sigma$ junto com a classe de equivalência da ordenação $v_{0}<v_{1}<$ $\ldots<v_{q}$ de seus vértices.

EXEMPlo 1.2.13. Seja $K$ um complexo simplicial abstrato. Se $q<0$, não existem $q$ simplexos orientados. Para todo vértice $v$ de $K$, existe um único 0-simplexo orientado $[v]$ e, para todo $q$-simplexo de $K$, com $q \geq 1$, correspondem exatamente dois simplexos $q$-orientados. Assim, se $\sigma$ for um 0 -simplexo, existe uma única orientação para $\sigma$. Uma orientação de um 1-simplexo é apenas uma escolha dos vértices inicial e final, a qual pode ser indicada esquematicamente, desenhando-se uma seta ao longo do simplexo. Uma orientação de um 2-simplexo é uma escolha de um sentido preferencial de rotação, a qual pode ser indicada por uma "seta circular"e, assim, sucessivamente.

DEFINIÇÃo 1.2.14. Dado um complexo simplicial abstrato $K$, seja $C_{q}(K ; R)$ o $R$-módulo livre cuja base são os $q$-simplexos orientados de $K$, chamado o $R$-módulo de $q$-cadeias simpliciais de $K$. Observemos que $C_{q}(K ; R)=\{0\}$, para $q<0$ ou $q>\operatorname{dim} K$ e, 
para todo $q \geq 0, C_{q}(K ; R)$ é o $R$-módulo livre cujo rank é igual ao número de $q$-simplexos orientados de $K$.

Para cada $q \geq 1$, definimos um operador bordo $\partial_{q}$ sobre um $q$-simplexo orientado pela fórmula:

$$
\partial_{q}[\sigma]=\partial_{q}\left[v_{0}, v_{1}, \ldots, v_{q}\right]=\sum_{m=0}^{q}(-1)^{m}\left[v_{0}, v_{1}, \ldots, \widehat{v_{m}}, \ldots, v_{q}\right],
$$

onde $\left[v_{0}, v_{1}, \ldots, \widehat{v_{m}}, \ldots, v_{q}\right]$ denota $o(q-1)$-simplexo obtido omitindo-se o vértice $v_{m}$. Então, $\partial_{q}$ se estende por $R$-linearidade a um único homomorfismo

$$
\partial_{q}: C_{q}(K ; R) \rightarrow C_{q-1}(K ; R)
$$

definido pela fórmula

$$
\partial_{q}\left(\sum_{i=1}^{l} r_{i}\left[\sigma_{i}\right]\right)=\sum_{i=1}^{l} r_{i} \partial_{q}\left[\sigma_{i}\right]=\sum_{i=1}^{l} r_{i}\left(\sum_{m=0}^{q}(-1)^{m}\left[v_{0}^{i}, v_{1}^{i}, \ldots, \widehat{v_{m}^{i}}, \ldots v_{q}^{i}\right]\right)
$$

Para $q \leq 0$, definimos $\partial_{q}: C_{q}(K ; R) \rightarrow C_{q-1}(K ; R)$ como sendo o homomorfismo trivial.

OBSERVAÇ̃̃o 1.2.15. O operador bordo $\partial_{q}$, dado na Definição 1.2.14, satisfaz $\partial_{q-1} \circ \partial_{q}=0$ (Vide [43, pg.30, Lema 5.3]). Portanto, existe um complexo de cadeias

$$
C(K ; R): \cdots \longrightarrow C_{q+1}(K ; R) \stackrel{\partial_{q+1}}{\longrightarrow} C_{q}(K ; R) \stackrel{\partial_{q}}{\longrightarrow} C_{q-1}(K ; R) \stackrel{\partial_{q-1}}{\longrightarrow} \cdots
$$

chamado complexo de cadeias orientado de $K$.

DEFINIÇÃo 1.2.16. Dado um par simplicial $(K, L)$, então $C_{q}(L ; R)$ é naturalmente um submódulo de $C_{q}(K ; R)$ e o $R$-módulo quociente

$$
C_{q}(K, L)=\frac{C_{q}(K ; R)}{C_{q}(L ; R)}
$$

é um $R$-módulo livre, chamado o $R$-módulo de $q$-cadeias simpliciais relativo de $K$ módulo $L$, o qual que tem como base todos os elementos da forma

$$
\sigma_{i}+C_{q}(L ; R)
$$

onde $\sigma_{i}$ é um $q$-simplexo orientado de $K$ que não está em $L$. Temos que

$$
\partial_{q}: C_{q}(K ; R) \rightarrow C_{q-1}(K ; R)
$$

satisfaz $\partial_{q}\left(C_{q}(L ; R)\right) \subset C_{q-1}(L ; R)$. Assim, $\partial_{q}: C_{q}(L ; R) \rightarrow C_{q-1}(L ; R)$ é a restrição do operador bordo. Logo, $\partial_{q}$ induz um $R$-homomorfismo

$$
C_{q}(K, L) \rightarrow C_{q-1}(K, L),
$$


chamado operador bordo relativo, o qual também será denotado por $\partial_{q}$ e satisfaz $\partial_{q-1} \circ \partial_{q}=0$. Dessa forma, obtemos um complexo de cadeias

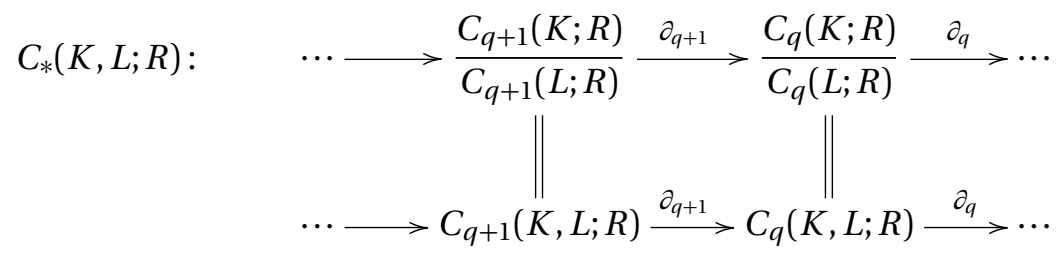

Definimos o $q$-ésimo $R$-módulo de $q$-cocadeias simpliciais relativo pela equação

$$
C^{q}(K, L ; R)=\operatorname{Hom}\left(C_{q}(K, L) ; R\right)=\operatorname{Hom}\left(\frac{C_{q}(K)}{C_{q}(L)} ; R\right) .
$$

O operador cobordo relativo $\delta^{q}: C^{q}(K, L ; R) \rightarrow C^{q+1}(K, L ; R)$ é definido como sendo o dual do operador bordo relativo $\partial_{q}: C_{q}(K, L) \rightarrow C_{q-1}(K, L)$ e, desde que satisfaz $\delta^{q}$ 。 $\delta^{q-1}=0$, obtemos um complexo de cocadeias,

$$
C^{*}(K, L ; R): \cdots \longrightarrow C^{q-1}(K, L ; R) \stackrel{\delta^{q-1}}{\longrightarrow} C^{q}(K, L ; R) \stackrel{\delta^{q}}{\longrightarrow} C^{q+1}(K, L ; R) \stackrel{\delta^{q+1}}{\longrightarrow} \cdots
$$

O q-ésimo $R$-módulo de cohomologia orientado do par simplicial $(K, L)$, com coeficientes em $R$, também chamado $R$-módulo de cohomologia simplicial e denotado por $H^{q}(K, L ; R)$ é definido como sendo

$$
H^{q}(K, L ; R)=\frac{\operatorname{ker} \delta^{q}}{\operatorname{Im} \delta^{q}}
$$

OBSERVAÇÃo 1.2.17. Dada uma aplicação simplicial de pares

$$
f:(K, L) \rightarrow\left(K_{1}, L_{1}\right),
$$

então para $q \geq 0, f$ induz um $R$-homomorfismo $f^{*}: H^{q}\left(K_{1}, L_{1} ; R\right) \rightarrow H^{q}(K, L ; R) . H^{*}(K, L ; R)$ e portanto $H^{q}$ é um funtor contravariante da categoria dos pares simpliciais na categoria dos $R$-módulos, o qual satisfaz os Axiomas de Eilenberg-Steenrod (Vide [43, Pg.265]).

\subsection{Os R-módulos de cohomologia de Čech relativos}

Nesta seção, apresentamos a construção dos $R$-módulos de cohomologia de Čech relativos. A idéia básica dessa construção é associar a um par de espaços topológicos $(X, A)$, pares simpliciais $\left(K_{\mathscr{U}}, L_{\mathscr{U}}\right)$. Os $R$-módulos de cohomologia de Čech do par $(X, A)$ serão definidos como sendo o limite direto dos $R$-módulos $H^{q}\left(K_{\mathscr{U}}, L_{\mathscr{U}} ; R\right)$. A referência básica para esta seção é o artigo Čech cohomology theory and the axioms de C.H.Dowker [14].

\subsubsection{O nervo de uma cobertura}

DEFINIÇ̃̃o 1.3.1. Seja $(X, A)$ um par de espaços topológicos. Uma cobertura aberta do $\boldsymbol{p a r}(X, A)$, a qual será denotada por $\left(\mathscr{U},\left.\mathscr{U}\right|_{A}\right)$, é uma coleção $\mathscr{U}$ de subconjuntos abertos de $X$ cuja reunião é $X$, juntamente com uma subcoleção $\left.\mathscr{U}\right|_{A}$ de $\mathscr{U}$, cuja reunião contém $A$. Denotaremos por $\Lambda(X, A)$ a coleção de todas as coberturas abertas do $\operatorname{par}(X, A)$. 
DEFINIÇÃo 1.3.2. Uma cobertura aberta $\left(\mathscr{V},\left.\mathscr{V}\right|_{A}\right)$ em $\Lambda(X, A)$ é chamada um refinamento aberto de $\left(\mathscr{U},\left.\mathscr{U}\right|_{A}\right)$, se todo conjunto aberto de $\mathscr{V}$ está contido em algum conjunto aberto de $\mathscr{U}$ e, se além disso, todo conjunto aberto de $\left.\mathscr{V}\right|_{A}$ está contido em algum conjunto aberto de $\left.\mathscr{U}\right|_{A}$.

DEFINIÇÃo 1.3.3. Definimos sobre $\Lambda(X, A)$ a seguinte relação de ordem:

$$
\left(\mathscr{U},\left.\mathscr{U}\right|_{A}\right) \leq\left(\mathscr{V},\left.\mathscr{V}\right|_{A}\right) \Leftrightarrow\left(\mathscr{V},\left.\mathscr{V}\right|_{A}\right) \text { é um refinamento de }\left(\mathscr{U},\left.\mathscr{U}\right|_{A}\right)
$$

LEMA 1.3.4. $(\Lambda(X, A), \leq)$ é um conjunto dirigido.

Demonstração: Para qualquer cobertura aberta $\left(\mathscr{U},\left.\mathscr{U}\right|_{A}\right) \operatorname{em} \Lambda(X, A)$, temos que $\left(\mathscr{U},\left.\mathscr{U}\right|_{A}\right) \leq$ $\left(\mathscr{U},\left.\mathscr{U}\right|_{A}\right)$. Além disso, se

$$
\left(\mathscr{U},\left.\mathscr{U}\right|_{A}\right) \leq\left(\mathscr{V},\left.\mathscr{V}\right|_{A}\right) \text { e }\left(\mathscr{V},\left.\mathscr{V}\right|_{A}\right) \leq\left(\mathscr{W},\left.\mathscr{W}\right|_{A}\right)
$$

dado $W \in \mathscr{W}$, existe $V \in \mathscr{V}$ com $W \subset V$. Para este $V$, existe $U \in \mathscr{U}$ tal que $W \subset V \subset U$. Logo, todo aberto de $\mathscr{W}$ está contido em algum aberto de $\mathscr{U}$. Analogamente, mostra-se que todo conjunto aberto de $\left.\mathscr{W}\right|_{A}$ está contido em algum aberto de $\left.\mathscr{U}\right|_{A}$. Portanto,

$$
\left(\mathscr{U},\left.\mathscr{U}\right|_{A}\right) \leq\left(\mathscr{W},\left.\mathscr{W}\right|_{A}\right)
$$

e, segue que, $(\Lambda(X, A), \leq)$ é um conjunto pré-ordenado. Para mostrarmos que $(\Lambda(X, A), \leq)$ é um conjunto dirigido, sejam $\left(\mathscr{U},\left.\mathscr{U}\right|_{A}\right)$ e $\left(\mathscr{V},\left.\mathscr{V}\right|_{A}\right)$ duas coberturas abertas em $(\Lambda(X, A)$ e consideremos

$$
\begin{aligned}
\mathscr{W} & =\{U \cap V ; U \in \mathscr{U} \text { e } V \in \mathscr{V}\} \\
\left.\mathscr{W}\right|_{A} & =\left\{\left.\left.U\right|_{A} \cap V\right|_{A} ;\left.\left.U\right|_{A} \in \mathscr{U}\right|_{A} \text { e }\left.\left.V\right|_{A} \in \mathscr{V}\right|_{A}\right\}
\end{aligned}
$$

Então, dado $W \in \mathscr{W}$ existem $U \in \mathscr{U}$ e $V \in \mathscr{V}$ tais que $W=U \cap V \subset U$ e $W=U \cap V \subset V$. Analogamente, mostra-se que todo aberto de $\left.\mathscr{W}\right|_{\mathscr{A}}$ está contido em algum aberto de $\left.\mathscr{U}\right|_{A}$ e em algum aberto de $\left.\mathscr{V}\right|_{A}$. Assim,

$$
\left(\mathscr{U},\left.\mathscr{U}\right|_{A}\right) \leq\left(\mathscr{W},\left.\mathscr{W}\right|_{A}\right) \text { e }\left(\mathscr{V},\left.\mathscr{V}\right|_{A}\right) \leq\left(\mathscr{W},\left.\mathscr{W}\right|_{A}\right)
$$

ou seja, $\left(\mathscr{W},\left.\mathscr{W}\right|_{A}\right)$ refina $\left(\mathscr{U},\left.\mathscr{U}\right|_{A}\right)$ e $\left(\mathscr{V},\left.\mathscr{V}\right|_{A}\right)$.

OBSERVAÇ̃̃o 1.3.5. Para simplificar a notação, de agora em diante, denotaremos uma cobertura $\left(\mathscr{U},\left.\mathscr{U}\right|_{A}\right)$ do $\operatorname{par}(X, A)$ simplesmente por $\mathscr{U}$, ou seja, $\mathscr{U}=\left(\mathscr{U},\left.\mathscr{U}\right|_{A}\right)$. Assim, a relação $\leq$ definida sobre $\Lambda(X, A)$ será representada da seguinte forma

$$
\mathscr{U}=\left(\mathscr{U},\left.\mathscr{U}\right|_{A}\right) \leq \mathscr{V}=\left(\mathscr{V},\left.\mathscr{V}\right|_{A}\right) \Leftrightarrow \mathscr{V} \text { refina } \mathscr{U}
$$

A cada cobertura aberta $\mathscr{U}=\left(\mathscr{U},\left.\mathscr{U}\right|_{A}\right)$ de um $\operatorname{par}(X, A)$, podemos associar um par simplicial $\left(K_{\mathscr{U}}, L_{\mathscr{U}}\right)$, de tal forma que os vértices de $K_{\mathscr{U}}$ são os conjuntos abertos dessa cobertura; um simplexo de $K_{\mathscr{U}}$ é qualquer coleção finita de conjuntos abertos de $\mathscr{U}$, cuja intersecção é não vazia. Um simplexo de $L_{\mathscr{U}}$ é qualquer coleção finita de conjuntos abertos de $\left.\mathscr{U}\right|_{A}$ cuja intersecção encontra $A$. Mais precisamente, temos a seguinte 
DEFINIÇÃo 1.3.6. Dada uma cobertura aberta $\mathscr{U}=\left(\mathscr{U},\left.\mathscr{U}\right|_{A}\right)$ do $\operatorname{par}(X, A)$, seja $K_{\mathscr{U}}$ o complexo simplicial cujos vértices são os conjuntos abertos $U \in \mathscr{U}$. Se $\sigma$ é um simplexo de $K_{\mathscr{U}}$, então $\sigma=\left[U_{0}, U_{1}, \ldots, U_{q}\right]$ é tal que

$$
U_{0} \cap U_{1} \cap \ldots \cap U_{q} \neq \emptyset .
$$

Além disso, $L_{\mathscr{U}}$ denotará o subcomplexo de $K_{\mathscr{U}}$ consistindo de todos os simplexos $\tau=$ $\left[U_{0}, U_{1}, \ldots, U_{n}\right]$ tais que

$$
U_{0} \cap U_{1} \cap \ldots \cap U_{n} \cap A \neq \emptyset .
$$

O par simplicial $\left(K_{\mathscr{U}}, L_{\mathscr{U}}\right)$ é chamado o nervo da cobertura $\mathscr{U}=\left(\mathscr{U},\left.\mathscr{U}\right|_{A}\right)$ e será denotado por $\mathscr{N}\left(\mathscr{U},\left.\mathscr{U}\right|_{A}\right)=\mathscr{N}(\mathscr{U})$. Para todo $q \geq 0$, os $R$-módulos de cohomologia simplicial

$$
H^{q}\left(K_{\mathscr{U}}, L_{\mathscr{U}} ; R\right)
$$

do par simplicial $\left(K_{\mathscr{U}}, L_{\mathscr{U}}\right)$ também são denotados por

$$
H^{q}(\mathscr{N}(\mathscr{U}) ; R)
$$

DEFINIÇÃo 1.3.7. Sejam $\mathscr{U}=\left(\mathscr{U},\left.\mathscr{U}\right|_{A}\right)$ e $\mathscr{V}=\left(\mathscr{V},\left.\mathscr{V}\right|_{A}\right)$ duas coberturas abertas do par $(X, A)$ tais que $\mathscr{U} \leq \mathscr{V}$, ou seja, $\mathscr{V}$ refina $\mathscr{U}$. Assim, para cada $V \in \mathscr{V}$, podemos escolher $U \in \mathscr{U}$ tal que $V \subset U$. Analogamente, para cada $\left.\left.V\right|_{A} \in \mathscr{V}\right|_{A}$, podemos escolher $\left.\left.U\right|_{A} \in \mathscr{U}\right|_{A}$ tal que $\left.\left.V\right|_{A} \subset U\right|_{A}$. Definimos uma aplicação, chamada aplicação projeção, da seguinte forma

$$
\begin{aligned}
f^{\mathscr{V} \mathscr{U}}:\left(K_{\mathscr{V}}, L_{\mathscr{V}}\right) & \rightarrow\left(K_{\mathscr{U}}, L \mathscr{U}\right) \\
V & \mapsto f^{\mathscr{V} \mathscr{U}}(V)=U, \operatorname{com} V \subset U
\end{aligned}
$$

LEMA 1.3.8. A aplicação projeção $f^{\mathscr{V} \mathscr{U}}:\left(K_{\mathscr{V}}, L_{\mathscr{V}}\right) \rightarrow\left(K_{\mathscr{U}}, L_{\mathscr{U}}\right)$ é uma aplicação simplicial.

Demonstração: Observemos inicialmente que $f^{\mathscr{V} \mathscr{U}}$ leva vértices de $K_{\mathscr{V}}$ em vértices de $K_{\mathscr{U}}$. Além disso, dado um simplexo $\sigma=\left[V_{0}, \ldots, V_{q}\right] \in K_{\mathscr{V}}$, temos

$$
\emptyset \neq V_{0} \cap \ldots \cap V_{q} \subset U_{0} \cap \ldots \cap U_{q}=f^{\mathscr{V} \mathscr{U}}\left(V_{0}\right) \cap \ldots \cap f^{\mathscr{V} \mathscr{U}}\left(V_{q}\right)
$$

e, deste modo, $f^{\mathscr{V} \mathscr{U}}(\sigma)=\left[f^{\mathscr{V} \mathscr{U}}\left(V_{0}\right), \ldots, f^{\mathscr{V} \mathscr{U}}\left(V_{q}\right)\right]$ é um simplexo em $K_{\mathscr{U}}$.

Mais ainda, dado um simplexo $\tau=\left[V_{0}, \ldots, V_{n}\right] \in L_{\mathscr{V}}$, então cada $\left.V_{i} \in \mathscr{V}\right|_{A}$ e, deste modo, $\left.f^{\mathscr{V} \mathscr{U}}\left(V_{i}\right) \in \mathscr{U}\right|_{A}$, para todo $i=0, \ldots, n$. Assim, temos que

$$
\emptyset \neq V_{0} \cap \ldots \cap V_{q} \cap A \subset U_{0} \cap \ldots \cap U_{n} \cap A=f^{\mathscr{V} \mathscr{U}}\left(V_{0}\right) \cap \ldots \cap f^{\mathscr{V} \mathscr{U}}\left(V_{n}\right) \cap A
$$

e, portanto, $f^{\mathscr{V} \mathscr{U}}(\tau)=\left[f^{\mathscr{V} \mathscr{U}}\left(V_{0}\right), \ldots, f^{\mathscr{V} \mathscr{U}}\left(V_{q}\right)\right]$ é um simplexo em $L \mathscr{U}$.

OBSERVAÇão 1.3.9. A aplicação projeção $f^{\mathscr{V} \mathscr{U}}:\left(K_{\mathscr{V}}, L_{\mathscr{V}}\right) \rightarrow\left(K_{\mathscr{U}}, L_{\mathscr{U}}\right)$ não é necessariamente única, desde que dado um aberto $V \in \mathscr{V}, f^{\mathscr{V} \mathscr{U}}(V)$ depende de uma escolha para o aberto $U \in \mathscr{U}$ satisfazendo a condição $V \subset U$. Entretanto, para cada $q \geq 0$, o homomorfismo induzido em cohomologia simplicial

$$
\left(f^{\mathscr{V} \mathscr{U}}\right)^{*}: H^{q}\left(K_{\mathscr{U}}, L_{\mathscr{U}} ; R\right) \rightarrow H^{q}\left(K_{\mathscr{V}}, L_{\mathscr{V}} ; R\right)
$$

é independente da escolha para $f^{\mathscr{V} \mathscr{U}}$, como mostra o lema a seguir. 
LEMA 1.3.10. [14, Pg. 182] Sejam $f^{\mathscr{V} \mathscr{U}}, g^{\mathscr{V} \mathscr{U}}:\left(K_{\mathscr{V}}, L_{\mathscr{V}}\right) \rightarrow\left(K_{\mathscr{U}}, L_{\mathscr{U}}\right)$ aplicações projeções obtidas a partir de escolhas distintas. Então, os homomorfismos induzidos em cohomologia simplicial coincidem, ou seja, para cada $q \geq 0$,

$$
\left(f^{\mathscr{V} \mathscr{U}}\right)^{*}=\left(g^{\mathscr{V} \mathscr{U}}\right)^{*}: H^{q}\left(K_{\mathscr{U}}, L_{\mathscr{U}} ; R\right) \rightarrow H^{q}\left(K_{\mathscr{V}}, L_{\mathscr{V}} ; R\right) .
$$

Obtemos assim uma coleção de homomorfismos

$$
\left\{\left(f^{\mathscr{V} \mathscr{U}}\right)^{*}: H^{q}\left(K_{\mathscr{U}}, L_{\mathscr{U}} ; R\right) \rightarrow H^{q}\left(K_{\mathscr{V}}, L_{\mathscr{V}} ; R\right) \text {, para cada } \mathscr{U} \leq \mathscr{V}\right\}
$$

e, dessa forma, podemos considerar a seguinte

PRoposição 1.3.11. Seja $\Lambda(X, A)$ a coleção de todas as coberturas abertas do par $(X, A)$. Então, para cada $q \geq 0$, a coleção

$$
\left\{H^{q}\left(K_{\mathscr{U}}, L_{\mathscr{U}} ; R\right),\left(f^{\mathscr{V} \mathscr{U}}\right)^{*}, \Lambda(X, A)\right\}
$$

é um sistema direto.

Demonstração: Dada uma aplicação projeção $f^{\mathscr{U} \mathscr{U}}:\left(K_{\mathscr{U}}, L \mathscr{U}\right) \rightarrow\left(K_{\mathscr{U}}, L \mathscr{U}\right)$, então $f^{\mathscr{U} \mathscr{U}}$ induz o homomorfismo identidade

$$
\left(f^{\mathscr{U} \mathscr{U}}\right)^{*}=\mathrm{Id}: H^{q}\left(K_{\mathscr{U}}, L_{\mathscr{U}} ; R\right) \rightarrow H^{q}\left(K_{\mathscr{U}}, L_{\mathscr{U}} ; R\right) .
$$

Se $\mathscr{U}, \mathscr{V}$ e $\mathscr{W}$ são coberturas abertas do $\operatorname{par}(X, A)$ tais que $\mathscr{U} \leq \mathscr{V} \leq \mathscr{W}$ e se

$$
f^{\mathscr{V} \mathscr{U}}:\left(K_{\mathscr{V}}, L_{\mathscr{V}}\right) \rightarrow\left(K_{\mathscr{U}}, L_{\mathscr{U}}\right) \text { e } f^{\mathscr{W} \mathscr{V}}:\left(K_{\mathscr{W}}, L_{\mathscr{W}}\right) \rightarrow\left(K_{\mathscr{V}}, L_{\mathscr{V}}\right)
$$

são aplicações projeções, então a composição

$$
f^{\mathscr{V} \mathscr{U}} \circ f^{\mathscr{W} \mathscr{V}}:\left(K_{\mathscr{W}}, L_{\mathscr{W}}\right) \rightarrow\left(K_{\mathscr{U}}, L_{\mathscr{U}}\right)
$$

é uma aplicação projeção, desde que dado $W \in \mathscr{W}$, existe $V=f^{\mathscr{W} \mathscr{V}}(W) \in \mathscr{V}$ tal que $W \subset$ $V$. Para este $V \in \mathscr{V}$, existe $U=f^{\mathscr{V} \mathscr{U}}(V) \in \mathscr{U}$, com $V \subset U$, logo existe $U=f^{\mathscr{V} \mathscr{U}} \circ f^{\mathscr{W} \mathscr{V}}(W)$, $\operatorname{com} W \subset U$.

Como $f^{\mathscr{W} \mathscr{U}}:\left(K_{\mathscr{W}}, L_{\mathscr{W}}\right) \rightarrow\left(K_{\mathscr{U}}, L_{\mathscr{U}}\right)$ é também uma aplicação projeção, segue do Lema 1.3.10 e do fato da cohomologia simplicial ser um funtor contravariante que

$$
\left(f^{\mathscr{W} \mathscr{U}}\right)^{*}=\left(f^{\mathscr{V} \mathscr{U}} \circ f^{\mathscr{W} \mathscr{V}}\right)^{*}=\left(f^{\mathscr{W} \mathscr{V}}\right)^{*} \circ\left(f^{\mathscr{V} \mathscr{U}}\right)^{*}: H^{q}\left(K_{\mathscr{U}}, L_{\mathscr{U}}\right) \rightarrow H^{q}\left(K_{\mathscr{W}}, L_{\mathscr{W}}\right) .
$$

Portanto, para cada $q \geq 0$, a coleção $\left\{H^{q}\left(K_{\mathscr{U}}, L_{\mathscr{U}} ; R\right),\left(f^{\mathscr{V} \mathscr{U}}\right)^{*}, \Lambda(X, A)\right\}$ é um sistema direto.

DEFINIÇÃo 1.3.12. O $q$-ésimo $R$-módulo de cohomologia de Čech do $\operatorname{par}(X, A)$, denotado por $\check{H}^{q}(X, A ; R)$, é definido como sendo o limite direto do sistema direto

$$
\left\{H^{q}\left(K_{\mathscr{U}}, L_{\mathscr{U}} ; R\right),\left(f^{\mathscr{V} \mathscr{U}}\right)^{*}, \Lambda(X, A)\right\}
$$

(Vide Definição 1.1.4), ou seja, para cada $q \geq 0$ :

$$
\check{H}^{q}(X, A ; R)=\lim _{\mathscr{U} \in \Lambda(X, A)} H^{q}\left(K_{\mathscr{U}}, L_{\mathscr{U}} ; R\right) .
$$




\subsubsection{O Homomorfismo induzido em Cohomologia de Čech}

Para cada aplicação de pares $f:(X, A) \rightarrow(Y, B)$, nosso objetivo é construir um sistema direto de $R$-homomorfismos, (Vide Definição 1.1.7) associado a $f$, o qual induz um $R$ homomorfismo entre os $R$-módulos de cohomologia de Čech. Para isto, sejam $\Lambda(Y, B)$ e $\Lambda(X, A)$ os conjuntos dirigidos formados pelas coleções de todas as coberturas abertas dos pares $(Y, B)$ e $(X, A)$, respectivamente. Dados os sistemas diretos de $R$-módulos

$$
\begin{aligned}
& \left\{H^{q}\left(K_{\mathscr{U}_{Y}}, L \mathscr{U}_{Y} ; R\right),\left(f^{\mathscr{V}_{Y} \mathscr{U}_{Y}}\right)^{*}, \Lambda(Y, B)\right\} \\
& \left\{H^{q}\left(K_{\mathscr{U}_{X}}, L_{\mathscr{U}_{X}} ; R\right),\left(f^{\mathscr{V}_{X} \mathscr{U}_{X}}\right)^{*}, \Lambda(X, A)\right\}
\end{aligned}
$$

seja $\phi: \Lambda(Y, B) \rightarrow \Lambda(X, A)$ definida por $\phi\left(\mathscr{U}_{Y}\right)=\phi\left(\mathscr{U}_{Y},\left.\mathscr{U}_{Y}\right|_{B}\right)=\left(\mathscr{U}_{X},\left.\mathscr{U}_{X}\right|_{A}\right)=\mathscr{U}_{X}$ onde as coleções são definidos da seguinte forma:

$$
\mathscr{U}_{X}=\left.\left\{f^{-1}(U): U \in \mathscr{U}_{Y}\right\} \quad \mathscr{U}_{X}\right|_{A}=\left\{f^{-1}\left(\left.U\right|_{B}\right):\left.\left.U\right|_{B} \in \mathscr{U}_{Y}\right|_{B}\right\} .
$$

LEMA 1.3.13. A aplicação $\phi: \Lambda(Y, B) \rightarrow \Lambda(X, A)$ definida em (1.13) preserva ordem.

Demonstração: Desde que $f(A) \subset B$, então $\phi$ está bem definida. Agora sejam $\mathscr{U}_{Y}, \mathscr{V}_{Y} \in$ $\Lambda(Y, B)$ tais que $\mathscr{U}_{Y} \leq \mathscr{V}_{Y}$, ou seja, $\mathscr{V}_{Y}$ refina $\mathscr{U}_{Y}$ e mostremos que $\phi\left(\mathscr{U}_{Y}\right)=\mathscr{U}_{X} \leq \mathscr{V}_{X}=$ $\phi\left(V_{Y}\right)$. Dado um aberto $V_{X} \in \mathscr{V}_{X}$, então $V_{X}=f^{-1}\left(V_{Y}\right)$, para algum $V_{Y} \in \mathscr{V}_{Y}$. Como $\mathscr{V}_{Y}$ refina $\mathscr{U}_{Y}$, existe um aberto $U_{Y} \in \mathscr{U}_{Y}$ tal que $V_{Y} \subset U_{Y}$. Assim,

$$
V_{X}=f^{-1}\left(V_{Y}\right) \subset f^{-1}\left(U_{Y}\right),
$$

e existe $U_{X}=f^{-1}\left(U_{Y}\right) \in \mathscr{U}_{X}$ tal que $V_{X} \subset U_{X}$. Portanto, $\mathscr{U}_{X} \leq \mathscr{V}_{X}$. Analogamente, mostrase que $\left.\mathscr{U}_{X}\right|_{A} \leq\left.\mathscr{V}_{X}\right|_{A}$.

Dadas duas coberturas abertas $\mathscr{U}_{Y} \in \Lambda(Y, B)$ e $\mathscr{U}_{X} \in \Lambda(X, A)$ tais que $\mathscr{U}_{X}=\phi\left(\mathscr{U}_{Y}\right)$, consideremos os nervos associados a estas coberturas,

$$
\left(K_{\phi\left(\mathscr{U}_{Y}\right)}, L_{\phi\left(\mathscr{U}_{Y}\right)}\right) \text { e }\left(K_{\mathscr{U}_{Y}}, L_{\mathscr{U}_{Y}}\right) .
$$

Seja $U_{X} \in \mathscr{U}_{X}=\phi\left(\mathscr{U}_{Y}\right)$ um vértice no complexo simplicial $K_{\mathscr{U}_{X}}=K_{\phi\left(\mathscr{U}_{Y}\right)}$. Então, $U_{X}=$ $f^{-1}\left(U_{Y}\right)$, para algum $U_{Y} \in \mathscr{U}_{Y}$, ou seja, $U_{Y}$ é um vértice em $K_{\mathscr{U}_{Y}}$. Analogamente, se $\left.U_{X}\right|_{A}$ é um vértice em $L \mathscr{U}_{X}=L_{\phi\left(\mathscr{U}_{Y}\right)}$, segue que $\left.U_{X}\right|_{A}=f^{-1}\left(\left.U_{Y}\right|_{B}\right)$, para algum vértice $\left.U_{Y}\right|_{B}$ em $L_{\mathscr{U}_{Y}}$. Observemos que o aberto $U_{X}$ não é necessariamente único, desde que dois conjuntos abertos em $\mathscr{U}_{Y}$ podem possuir a mesma imagem inversa pela aplicação $f$. Contudo, fixamos uma escolha para $U_{X}$ e definimos a aplicação

$$
\begin{array}{cl}
f_{\mathscr{U}_{Y}}:\left(K_{\phi\left(\mathscr{U}_{Y}\right)}, L_{\phi\left(\mathscr{U}_{Y}\right)}\right) & \rightarrow\left(K_{\mathscr{U}_{Y}}, L_{\mathscr{U}_{Y}}\right), \\
U_{X} & \mapsto U_{Y}, \quad \operatorname{comde} \phi\left(\mathscr{U}_{Y}\right)=\mathscr{U}_{X} \\
U_{X}=f^{-1}\left(U_{Y}\right)
\end{array}
$$

a qual leva os vértices $U_{X}=f^{-1}\left(U_{Y}\right)$ de $K_{\phi\left(\mathscr{U}_{Y}\right)}$ sobre os vértices $U_{Y}$ de $K_{\mathscr{U}_{Y}}$. Mostremos que $f_{\mathscr{U}_{Y}}$ assim definida é uma aplicação simplicial. Com efeito, seja $\sigma=\left[U_{X}^{0}, \ldots, U_{X}^{p}\right]$ um simplexo em $K_{\mathscr{U}_{X}}=K_{\phi\left(\mathscr{U}_{Y}\right)}$. Desde que

$$
\emptyset \neq U_{X}^{0} \cap \ldots \cap U_{X}^{p}=f^{-1}\left(U_{Y}^{0}\right) \cap \ldots \cap f^{-1}\left(U_{Y}^{p}\right)=f^{-1}\left(U_{Y}^{0} \cap \cdots \cap U_{Y}^{p}\right),
$$


isso implica que $U_{Y}^{0} \cap \cdots \cap U_{Y}^{p} \neq \emptyset$. Portanto,

$$
f_{\mathscr{U}_{Y}}(\sigma)=f_{\mathscr{U}_{Y}}\left(\left[U_{X}^{0}, \ldots, U_{X}^{p}\right]\right)=\left[U_{Y}^{0}, \cdots, U_{Y}^{p}\right]
$$

é um simplexo de $K_{\mathscr{U}_{Y}}$. Mais ainda, dado um simplexo $\tau=\left[U_{X}^{0}, \ldots, U_{X}^{n}\right] \in L_{\mathscr{U}_{X}}=L_{\phi\left(\mathscr{U}_{Y}\right)}$, então cada $\left.U_{X}^{i} \in \mathscr{U}_{X}\right|_{A}$ e, como $f(A) \subset B$, deste modo, $f_{\mathscr{U}_{Y}}\left(U_{X}^{i}\right)=\left.U_{Y}^{i} \in \mathscr{U}_{Y}\right|_{B}$, para todo $i=0, \ldots, n$. Assim, temos que

$$
\begin{aligned}
\emptyset \neq U_{X}^{0} \cap \ldots \cap U_{X}^{n} \cap A & \subset f^{-1}\left(U_{Y}^{0}\right) \cap \ldots \cap f^{-1}\left(U_{Y}^{n}\right) \cap f^{-1}(B) \\
& =f^{-1}\left(U_{Y}^{0} \cap \cdots \cap U_{Y}^{n} \cap B\right)
\end{aligned}
$$

isso implica que $U_{Y}^{0} \cap \cdots \cap U_{Y}^{n} \cap B \neq \emptyset$. Portanto,

$$
f_{\mathscr{U}_{Y}}(\tau)=f_{\mathscr{U}_{Y}}\left(\left[U_{X}^{0}, \ldots, U_{X}^{n}\right]\right)=\left[U_{Y}^{0}, \cdots, U_{Y}^{n}\right]
$$

é um simplexo de $L_{\mathscr{U}_{Y}}$.

OBSERVAÇ̃̃o 1.3.14. A aplicação $f_{\mathscr{U}_{Y}}:\left(K_{\phi\left(\mathscr{U}_{Y}\right)}, L_{\phi\left(\mathscr{U}_{Y}\right)}\right) \rightarrow\left(K_{\mathscr{U}_{Y}}, L_{\mathscr{U}_{Y}}\right)$, onde $\phi\left(\mathscr{U}_{Y}\right)=\mathscr{U}_{X}$, definida em (1.14), não é necessariamente única, desde que dado um aberto $U_{X} \in \mathscr{U}_{X}$, $f_{\mathscr{U}_{Y}}\left(U_{X}\right)$ depende de uma escolha para o aberto $U_{Y} \in \mathscr{U}_{Y}$ satisfazendo a condição $U_{X}=$ $f^{-1}\left(U_{Y}\right)$. Entretanto, para cada $q \geq 0$, o homomorfismo induzido em cohomologia simplicial

$$
\left(f_{\mathscr{U}_{Y}}\right)^{*}: H^{q}\left(K_{\mathscr{U}_{Y}}, L \mathscr{U}_{Y} ; R\right) \rightarrow H^{q}\left(K_{\phi\left(\mathscr{U}_{Y}\right)}, L_{\phi\left(\mathscr{U}_{Y}\right)} ; R\right) ; \phi\left(\mathscr{U}_{Y}\right)=\mathscr{U}_{X}
$$

é independente da escolha para $f_{\mathscr{U}_{Y}}$, como mostra o lema a seguir.

LEMA 1.3.15. [14, Pg. 283] Sejam $\left.f_{\mathscr{U}_{Y}}, g_{\mathscr{U}_{Y}}:\left(K_{\phi\left(\mathscr{U}_{Y}\right)}, L_{\phi(\mathscr{U} Y}\right)\right) \rightarrow\left(K_{\mathscr{U}_{Y}}, L_{\mathscr{U}_{Y}}\right)$ aplicações obtidas a partir de escolhas distintas. Então, os homomorfismos induzidos em cohomologia simplicial coincidem, ou seja, para cada $q \geq 0$,

$$
\left(f_{\mathscr{U}_{Y}}\right)^{*}=\left(g_{\mathscr{U}_{Y}}\right)^{*}: H^{q}\left(K_{\mathscr{U}_{Y}}, L_{\mathscr{U}_{Y}} ; R\right) \rightarrow H^{q}\left(K_{\phi\left(\mathscr{U}_{Y}\right)}, L_{\phi\left(\mathscr{U}_{Y}\right)} ; R\right) .
$$

Obtemos assim, uma coleção de $R$-homomorfismos

$$
\left\{\left(f_{\mathscr{U}_{Y}}\right)^{*}: H^{q}\left(K_{\mathscr{U}_{Y}}, L_{\mathscr{U}_{Y}} ; R\right) \rightarrow H^{q}\left(K_{\phi\left(\mathscr{U}_{Y}\right)}, L_{\phi\left(\mathscr{U}_{Y}\right)} ; R\right), \mathscr{U}_{Y} \in \Lambda(Y, B)\right\}
$$

e, dessa forma, podemos considerar a seguinte

PROPOSIÇÃo 1.3.16. Dada uma aplicação de pares $f:(X, A) \rightarrow(Y, B)$, consideremos os sistemas diretos de R-módulos:

$$
\begin{aligned}
& \left\{H^{q}\left(K_{\mathscr{U}_{Y}}, L_{\mathscr{U}_{Y}} ; R\right),\left(f^{\mathscr{V}_{Y} \mathscr{U}_{Y}}\right)^{*}, \Lambda(Y, B)\right\}, \\
& \left\{H^{q}\left(K_{\mathscr{U}_{X}}, L_{\mathscr{U}_{X}} ; R\right),\left(f^{\mathscr{V}_{X} \mathscr{U}_{X}}\right)^{*}, \Lambda(X, A)\right\},
\end{aligned}
$$

com $\mathscr{U}_{X}=\phi\left(\mathscr{U}_{Y}\right) \leq \phi\left(\mathscr{V}_{Y}\right)=\mathscr{V}_{X}$, para cada $\mathscr{U}_{Y} \leq \mathscr{V}_{Y}$ (Vide Lema 1.3.13), onde $\phi: \Lambda(Y, B) \rightarrow \Lambda(X, A)$ é a aplicação definida em (1.13), a qual preserva ordem. Então, $a$ familia de R-homomorfismos

$$
\left\{\left(f_{\mathscr{U}_{Y}}\right)^{*}: H^{q}\left(K_{\mathscr{U}_{Y}}, L_{\mathscr{U}_{Y}} ; R\right) \rightarrow H^{q}\left(K_{\phi\left(\mathscr{U}_{Y}\right)}, L_{\phi\left(\mathscr{U}_{Y}\right)} ; R\right), \mathscr{U}_{Y} \in \Lambda(Y, B)\right\},
$$

é um sistema direto de R-homomorfismos. 
Demonstração: Consideremos o seguinte diagrama

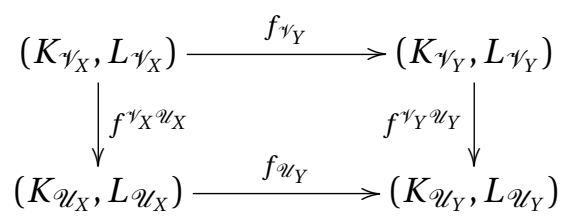

onde $\mathscr{U}_{X}=\phi\left(\mathscr{U}_{Y}\right) \leq \phi\left(\mathscr{V}_{Y}\right)=\mathscr{V}_{X}$, para cada $\mathscr{U}_{Y} \leq \mathscr{V}_{Y}$ em $\Lambda(Y, B)$. Dado um aberto $V_{X} \in$ $\mathscr{V}_{X}=\phi\left(\mathscr{V}_{Y}\right)$, então $V_{X}=f^{-1}\left(V_{Y}\right)$, para algum $V_{Y} \in \mathscr{V}_{Y}$. Como $\mathscr{V}_{Y}$ refina $\mathscr{U}_{Y}$, existe um aberto $U_{Y} \in \mathscr{U}_{Y}$ tal que $V_{Y} \subset U_{Y}$. Assim,

$$
V_{X}=f^{-1}\left(V_{Y}\right) \subset f^{-1}\left(U_{Y}\right)
$$

e existe $U_{X}=f^{-1}\left(U_{Y}\right) \in \mathscr{U}_{X}$ tal que $V_{X} \subset U_{X}$. Então, segue das Definições (1.9) e (1.14) que

$$
\begin{aligned}
\left(f^{\mathscr{V}_{Y} \mathscr{U}_{Y}} \circ f_{\mathscr{V}_{Y}}\right)\left(V_{X}\right) & =f^{\mathscr{V _ { Y }} \mathscr{U}_{Y}}\left(f_{\mathscr{V}_{Y}}\left(V_{X}\right)\right) \\
& =f^{\mathscr{V _ { Y }} \mathscr{U}_{Y}}\left(V_{Y}\right) \\
& =U_{Y} \\
& =f_{\mathscr{U}_{Y}}\left(U_{X}\right) \\
& =f_{\mathscr{U}_{Y}}\left(f^{\mathscr{V}_{X} \mathscr{U}_{X}}\left(V_{X}\right)\right) \\
& =\left(f_{\mathscr{U}_{Y}} \circ f^{\mathscr{V}_{X} \mathscr{U}_{X}}\right)\left(V_{X}\right) .
\end{aligned}
$$

Deste modo, segue que o diagrama (1.16) é comutativo. Assim, temos que

$$
\begin{aligned}
\left(f_{\mathscr{V}_{Y}}\right)^{*} \circ\left(f^{\mathscr{V}_{Y} \mathscr{U}_{Y}}\right)^{*} & =\left(f^{\mathscr{V}_{Y} \mathscr{U}_{Y}} \circ f_{\mathscr{V}_{Y}}\right)^{*} \\
& =\left(f_{\mathscr{U}_{Y}} \circ f^{\mathscr{V}} \mathscr{U}_{X}\right)^{*} \\
& =\left(f^{\mathscr{V}_{X} \mathscr{U}_{X}}\right)^{*} \circ\left(f_{\mathscr{U}_{Y}}\right)^{*} .
\end{aligned}
$$

e, portanto, o correspondente diagrama em cohomologia

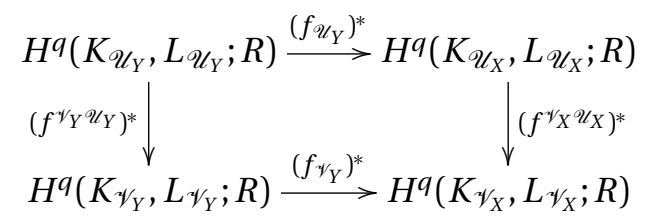

é comutativo. Portanto, a familia de $R$-homomorfismos dada é um sistema direto de $R$-homomorfismos.

DEFINIÇÃo 1.3.17. Seja $f:(X, A) \rightarrow(Y, B)$ uma aplicação de pares. Definimos o homomorfismo induzido em cohomologia de $\check{C}$ ech, o qual será denotado por $\check{f}^{*}$, como sendo o limite direto do sistema direto de $R$-homomorfismos

$$
\left\{\left(f_{\mathscr{U}_{Y}}\right)^{*}: H^{q}\left(K_{\mathscr{U}_{Y}}, L_{\mathscr{U}_{Y}} ; R\right) \rightarrow H^{q}\left(K_{\phi\left(\mathscr{U}_{Y}\right)}, L_{\phi\left(\mathscr{U}_{Y}\right)} ; R\right), \mathscr{U}_{Y} \in \Lambda(Y, B)\right\},
$$

ou seja, para cada $q \geq 0$ :

$$
\check{f}^{*}=\lim _{\mathscr{U}_{Y} \in \Lambda(Y, B)}\left(f_{\mathscr{U}_{Y}}\right)^{*}: \check{H}^{q}(Y, B ; R) \rightarrow \check{H}^{q}(X, A ; R)
$$




\subsubsection{Os Axiomas de Eilenberg-Steenrod}

Denotemos por Top ${ }^{2}$ a categoria dos pares topológicos junto com as aplicações contínuas de pares e por R-mod a categoria que tem como objetos os $R$-módulos e como morfismos os $R$-homomorfismos.

TEOREMA 1.3.18. Para todo $q \geq 0$, a função

$$
\check{H}^{q}: \boldsymbol{T o p}{ }^{2} \rightarrow \boldsymbol{R}-\boldsymbol{m o d}
$$

que associa a cada par de espaços $(X, A)$, o R-módulo de cohomologia de Čech

$$
\check{H}^{q}(X, A ; R)
$$

e a cada aplicação continua de pares $f:(X, A) \rightarrow(Y, B)$ associa o homomorfismo induzido em cohomologia de Čech

$$
\check{f}^{*}: \check{H}^{q}(Y, B ; R) \rightarrow \check{H}^{q}(X, A ; R)
$$

é um funtor contravariante, chamado q-funtor Cohomologia de Čech.

Demonstração: [1] Seja Id : $(X, A) \rightarrow(X, A)$ a aplicação identidade. Então, para cada

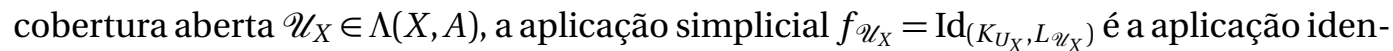
tidade e, portanto, induz o homomorfismo identidade $\left(f_{\mathscr{U}_{X}}\right)^{*}$ na cohomologia simplicial e, deste modo,

$$
\check{f}^{*}=(\check{I} d)^{*}: \check{H}^{q}(X, A ; R) \rightarrow \check{H}^{q}(X, A ; R) .
$$

[2] Sejam $f:(X, A) \rightarrow(Y, B), g:(Y, B) \rightarrow(Z, C)$ aplicações de pares. Para cada cobertura aberta $\mathscr{U}_{Z} \in \Lambda(Z, C)$, seja $\mathscr{U}_{Y}=g^{-1}\left(\mathscr{U}_{Z}\right), \mathscr{U}_{X}=f^{-1}\left(\mathscr{U}_{Y}\right)$. Consideremos

$$
\begin{gathered}
f_{\mathscr{U}_{Y}}:\left(K_{\mathscr{U}_{X}}, L_{\mathscr{U}_{X}}\right) \rightarrow\left(K_{\mathscr{U}_{Y}}, L_{\mathscr{U}_{Y}}\right) \quad, g_{\mathscr{U}_{Z}}:\left(K_{\mathscr{U}_{Y}}, L_{\mathscr{U}_{Y}}\right) \rightarrow\left(K_{\mathscr{U}_{Z}}, L \mathscr{U}_{Z}\right) \\
(g \circ f)_{\mathscr{U}_{Z}}:\left(K_{\mathscr{U}_{X}}, L \mathscr{U}_{X}\right) \rightarrow\left(K_{\mathscr{U}_{Z}}, L \mathscr{U}_{Z}\right)
\end{gathered}
$$

as aplicações simpliciais. Se $U_{X}$ é um vértice de $K_{\mathscr{U}_{X}}$, então $f_{\mathscr{U}_{Y}}\left(U_{X}\right)$ é um vértice $V_{Y}$ de $K_{\mathscr{U}_{Y}}$ tal que $U_{X}=f^{-1}\left(V_{Y}\right)$ e $g_{\mathscr{U}_{Z}}\left(V_{Y}\right)$ é um vértice $W_{Z}$ de $K_{\mathscr{U}_{Z}}$ tal que $V_{Y}=g^{-1}\left(W_{Z}\right)$. Assim, $g_{\mathscr{U}_{Z}} \circ f_{\mathscr{U}_{Y}}$ leva o vértice $U_{X}$ no vértice $W_{Z}$ e $U_{X}=(g \circ f)^{-1}\left(W_{Z}\right)$. Segue do Lema $1.3 .15 \mathrm{e}$ do fato da cohomologia simplicial ser um função contravariante que

$$
\left((g \circ f)_{\mathscr{U}_{Z}}\right)^{*}=\left(g_{\mathscr{U}_{Z}} \circ f_{\mathscr{U}_{Y}}\right)^{*}=\left(f_{\mathscr{U}_{Y}}\right)^{*} \circ\left(g_{\mathscr{U}_{Z}}\right)^{*}
$$

e, portanto, usando a Observação 1.1.11 temos que

$$
\begin{aligned}
(g \circ f)^{*} & =\lim _{\overrightarrow{\mathscr{U}_{Z} \in \Lambda(Z, C)}}\left((g \circ f)_{\mathscr{U}_{Z}}\right)^{*} \\
& =\lim _{\overrightarrow{\mathscr{U}_{Z} \in \Lambda(Z, C)}}\left[\left(f_{\mathscr{U}_{Y}}\right)^{*} \circ\left(g_{\mathscr{U}_{Z}}\right)^{*}\right] \\
& =\left(\lim _{\mathscr{U}_{Y} \in \Lambda(Y, B)}\left(f_{\mathscr{U}_{Y}}\right)^{*}\right)\left(\lim _{\mathscr{U}_{Z} \in \hat{\Lambda}(Z, C)}\left(g_{\mathscr{U}_{Z}}\right)^{*}\right) \\
& =\check{f}^{*} \circ \check{g}^{*},
\end{aligned}
$$


o que implica que $\check{H}^{q}$ é um funtor contravariante.

C.H. Dowker mostrou em [14, pgs. 285-292], que a Teoria de Cohomologia de Čech satisfaz os axiomas de Eilenberg-Steenrod. Em resumo, a Teoria de Cohomologia de Čech é a coleção dos $q$-funtores cohomologia de Čech $\left\{\check{H}^{q}\right\}$ que satisfaz os seguintes axiomas:

1. (InVARiÂnCIA HomotóPICA) Se $f_{1}, f_{2}:(X, A) \rightarrow(Y, B)$ são aplicações homotópicas, então $\check{f}_{1}^{*}=\check{f}_{2}^{*}$.

2. (Exatid ̃̃o) Dados os pares $(X, A)$ e $(A, B)$, existe um $R$-homomorfismo

$$
\delta: \check{H}^{q}(A, B ; R) \rightarrow \check{H}^{q+1}(X, A ; R)
$$

que comuta com os homomorfismos induzidos em cohomologia de Čech e a sequência longa

$$
\cdots \stackrel{\delta}{\longrightarrow} \check{H}^{q+1}(X, A ; R) \stackrel{\check{J}^{*}}{\longrightarrow} \check{H}^{q+1}(X, B ; R) \stackrel{\check{I}^{*}}{\longrightarrow} \check{H}^{q+1}(A, B ; R) \stackrel{\delta}{\longrightarrow}
$$

é exata, onde $\imath:(A, B) \rightarrow(X, B), \mathrm{J}:(X, B) \rightarrow(X, A)$ são as inclusões.

3. (Excisão) Se $(X, A)$ é um par e $B$ é um subconjunto de $X$ tal que $\bar{B} \subset \operatorname{Int}(A)$, então, para todo $q \geq 0$, o homomorfismo

$$
\check{\imath}^{*}: \check{H}^{q}(X, A ; R) \rightarrow \check{H}^{q}(X-B, A-B ; R)
$$

induzido da inclusão é um isomorfismo.

4. (Dimensão) Se $X=\left\{x_{0}\right\}$ consiste de um apenas ponto, então

$$
\check{H}^{q}\left(\left\{x_{0}\right\} ; R\right)=\left\{\begin{array}{ll}
R, & q=0 \\
0, & q>0
\end{array} .\right.
$$

Segue da validade dos Axiomas de Eilenberg-Steenrod , como acontece no caso da cohomologia singular, que existe a sequência de Mayer-Vietoris na cohomologia de Čech, dada a seguir:

PROPOSIÇão 1.3.19. [49, Corolario 9, pag. 239] Se $\left\{\left(X_{1}, A_{1}\right),\left(X_{2}, A_{2}\right)\right\}$ for um par excisivo ${ }^{4}$ de $(X, A)=\left(X_{1} \cup X_{2}, A_{1} \cup A_{2}\right)$, então a seguinte sequência longa

$$
\cdots \rightarrow \check{H}^{q}(X, A ; R) \stackrel{\zeta}{\rightarrow} \check{H}^{q}\left(X_{1}, A_{1} ; R\right) \oplus \check{H}^{q}\left(X_{2}, A_{2} ; R\right) \stackrel{\eta}{\rightarrow} \check{H}^{q}\left(X_{1} \cap X_{2}, A_{1} \cap A_{2} ; R\right) \rightarrow \cdots
$$

é exata, onde $\zeta=\left(\check{i}_{1}^{*},-\check{i}_{2}^{*}\right), \eta=\check{\ell}_{1}^{*}+\check{\ell}_{2}^{*}$ com $i_{1}:\left(X_{1}, A_{1}\right) \rightarrow(X, A), i_{2}:\left(X_{2}, A_{2}\right) \rightarrow(X, A)$, $\ell_{1}:\left(X_{1} \cap X_{2}, A_{1} \cap A_{2}\right) \rightarrow\left(X_{1}, A_{1}\right) e \ell_{2}:\left(X_{1} \cap X_{2}, A_{1} \cap A_{2}\right) \rightarrow\left(X_{2}, A_{2}\right)$.

\footnotetext{
${ }^{4}$ Vide [49, Pag. 188]
} 
DEFINIÇÃo 1.3.20. Dizemos que o par $(U, V)$ é um par de vizinhanças do par $(X, A)$ se $U$ for uma vizinhança de $X$ e se $V$ for uma vizinhança de $A$. Se $U$ e $V$ forem vizinhanças fechadas em um espaço topológico $Y$, dizemos que $(U, V)$ é um par de vizinhanças fechadas.

Seja $\mathscr{N}$ a família de pares de vizinhanças fechadas $\{(U, V)\}$ do par $(X, A)$ com a seguninte relação de ordem dada pelas inclusões, ou seja,

$$
(U, V) \leq\left(U^{\prime}, V^{\prime}\right) \Leftrightarrow U^{\prime} \subset U \text { e } V^{\prime} \subset V .
$$

Temos que $\mathscr{N}$ conjunto é um dirigido. Com efeito, para todo $(U, V) \in \mathscr{N}$,

$$
(U, V) \leq(U, V)
$$

pois $U \subset U$ e $V \subset V$. Dados $(U, V) \leq\left(U^{\prime}, V^{\prime}\right) \leq\left(U^{\prime \prime}, V^{\prime \prime}\right)$, então temos as inclusões $V^{\prime \prime} \subset$ $V^{\prime} \subset V$ e $U^{\prime \prime} \subset U^{\prime} \subset U$, o que implica que $V^{\prime \prime} \subset V$ e $U^{\prime \prime} \subset U$, logo, $(U, V) \leq\left(U^{\prime \prime}, V^{\prime \prime}\right)$. Finalmente, dados $(U, V),\left(U^{\prime}, V^{\prime}\right)$ pares de vizinhanças fechadas de $(X, A)$, temos que $U \cap U^{\prime}$ é uma vizinhança fechada que está contida em $U$ e $U^{\prime}$ e, analogamente $V \cap V^{\prime}$ é uma vizinhança fechada que está contida em $V$ e $V^{\prime}$ e, portanto,

$$
(U, V) \leq\left(U \cap U^{\prime}, V \cap V^{\prime}\right) \quad\left(U^{\prime}, V^{\prime}\right) \leq\left(U \cap U^{\prime}, V \cap V^{\prime}\right)
$$

ou seja $\mathscr{N}$ é um conjunto dirigido. Usando as propriedades da cohomologia de Čech, temos que a família

$$
\left\{\check{H}^{q}(U, V), \check{l}^{*}, \mathscr{N}\right\}
$$

onde $\check{\imath}^{*}: \check{H}^{q}\left(U^{\prime}, V^{\prime} ; R\right) \rightarrow \check{H}^{q}(U, V ; R)$ é a induzida em cohomologia de Čech da inclusão $\imath:\left(U^{\prime}, V^{\prime}\right) \rightarrow(U, V)$, é um sistema direto. Deste modo, temos o seguinte

TEOREMA 1.3.21. [53, Teo. 6-16 pag 227 e Teo. 7.10 pag. 250]

(Teorema da Continuidade) Seja $(X, A)$ um par de espaços, onde A é fechado em X e X é um subconjunto fechado de um espaço normal $Y$. Então, existe um isomorfismo

$$
\varpi: \lim _{(U, V) \in \mathcal{N}} \check{H}^{q}(U, V ; R) \rightarrow \check{H}^{q}(X, A ; R)
$$

ou seja,

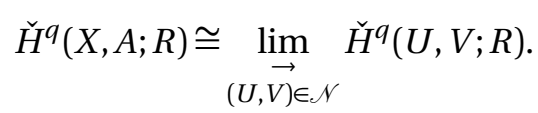

\subsubsection{Produto cup $\smile$}

Sejam $\left(X, A_{1}\right),\left(X, A_{2}\right)$ pares de espaços tais que $\left\{A_{1}, A_{2}\right\}$ é um par excisivo de $A_{1} \cup A_{2}$ e $\Lambda(X, A)$ é o conjunto dirigido de todas coberturas abertas do $\operatorname{par}(X, A)$. Suponhamos que $\left(K_{\mathscr{U}}, L_{\mathscr{U}}^{1}\right),\left(K_{\mathscr{U}}, L_{\mathscr{U}}^{2}\right)$ e $\left(K_{\mathscr{U}}, L_{\mathscr{U}}^{12}\right)$ sejam os pares simpliciais para $\left(X, A_{1}\right),\left(X, A_{2}\right)$ e $\left(X, A_{1} \cup\right.$ $\left.A_{2}\right)$, respectivamente. 
Temos que, se $\left\{A_{1}, A_{2}\right\}$ é um par excisivo de $A_{1} \cup A_{2}$, então $\left\{L_{\mathscr{U}}^{1}, L_{\mathscr{U}}^{2}\right\}$ é um par excisivo de $L_{\mathscr{U}}^{12}$ e, deste modo temos o seguinte diagrama comutativo

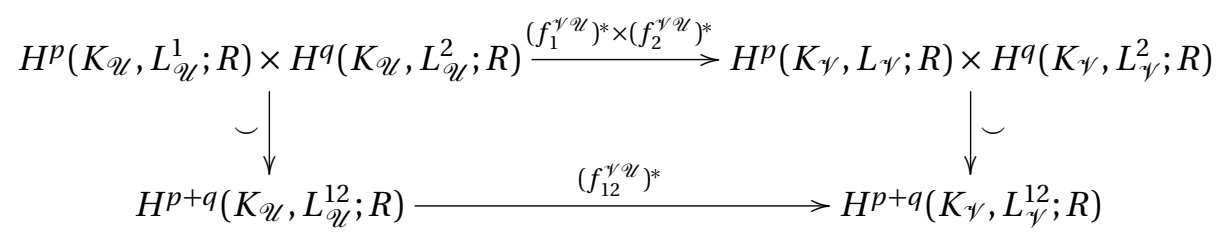

com $\mathscr{V} \leq \mathscr{U}$, onde $\smile$ é o produto cup na cohomologia simplicial. Com efeito, dados $x \in H^{p}\left(K_{\mathscr{U}}, L_{\mathscr{U}}^{1} ; R\right)$ e $y \in H^{q}\left(K_{\mathscr{U}}, L_{\mathscr{U}}^{2} ; R\right)$

$$
\begin{aligned}
\left(f_{12}^{\mathscr{V} \mathscr{U}}\right)^{*}(\smile(x, y)) & =\left(f_{12}^{\mathscr{V} \mathscr{U}}\right)^{*}(x \smile y) \\
& =\left(f_{1}^{\mathscr{V} \mathscr{U}}\right)^{*}(x) \smile\left(f_{2}^{\mathscr{V} \mathscr{U}}\right)^{*}(y) \\
& =\smile\left(\left(f_{1}^{\mathscr{V} \mathscr{U}}\right)^{*}(x),\left(f_{2}^{\mathscr{V} \mathscr{U}}\right)^{*}(y)\right) \\
& =\smile\left[\left(f_{1}^{\mathscr{V} \mathscr{U}}\right)^{*} \times\left(f_{2}^{\mathscr{V} \mathscr{U}}\right)^{*}\right](x, y) .
\end{aligned}
$$

Assim, o homomorfismo produto cup $\smile$ em cohomologia simplicial

$$
\smile: H^{p}\left(K_{\mathscr{U}}, L_{\mathscr{U}}^{1} ; R\right) \times H^{q}\left(K_{\mathscr{U}}, L_{\mathscr{U}}^{2} ; R\right) \rightarrow H^{p+q}\left(K_{\mathscr{U}}, L_{\mathscr{U}}^{12} ; R\right)
$$

é um homomorfismo de sistemas diretos.

DEFINIÇ̃̃o 1.3.22. Sejam $\left(X, A_{1}\right),\left(X, A_{2}\right)$ pares topológicos tais que $\left\{A_{1}, A_{2}\right\}$ é um par excisivo de $A_{1} \cup A_{2}$. O homomorfismo induzido do homomorfismo de limites diretos, denotado por $\smile$, é chamado produto cup na cohomologia de Čech, ou seja

$$
\smile: \check{H}^{p}\left(X, A_{1} ; R\right) \otimes \check{H}^{q}\left(X, A_{2} ; R\right) \rightarrow \check{H}^{p+q}\left(X, A_{1} \cup A_{2} ; R\right)
$$

O produto cup na cohomologia de Čech, satisfaz as seguintes propriedades:

PROPOSIÇão 1.3.23.

1. (Linearidade) Sejam $f:\left(X, A_{1} \cup A_{2}\right) \rightarrow\left(Y, B_{1} \cup B_{2}\right), f_{1}:\left(X, A_{1}\right) \rightarrow\left(Y, B_{1}\right)$ e $f_{2}:$ $\left(X, A_{2}\right) \rightarrow\left(Y, B_{2}\right)$ aplicações de pares tais que, para todo $x \in X, f_{1}(x)=f_{2}(x)=f(x)$. Suponhamos que $u \in \check{H}^{p}\left(Y, B_{1} ; R\right)$ e $v \in \check{H}^{q}\left(Y, B_{2} ; R\right)$, então em $\check{H}^{p+q}\left(X, A_{1} \cup A_{2} ; R\right)$ temos

$$
\check{f}^{*}(u \smile v)=\check{f}_{1}^{*}(u) \smile \check{f}_{2}^{*}(v) .
$$

2. (Identidade) Para qualquer $u \in \check{H}^{q}(X, A ; R)$, temos que

$$
1 \smile u=u \smile 1=u, \operatorname{com} 1 \in \check{H}^{0}(X ; R) .
$$

3. (Associatividade) Sejam $u_{1} \in \check{H}^{p}\left(X, A_{1} ; R\right), u_{2} \in \check{H}^{q}\left(X, A_{2} ; R\right) e u_{3} \in \check{H}^{r}\left(X, A_{3} ; R\right)$, então em $\check{H}^{p+q+r}\left(X, A_{1} \cup A_{2} \cup A_{3} ; R\right)$ temos

$$
u_{1} \smile\left(u_{2} \smile u_{3}\right)=\left(u_{1} \smile u_{2}\right) \smile u_{3} .
$$


4. (Anticomutatividade) Sejam $u \in \check{H}^{p}\left(X, A_{1} ; R\right)$ e $v \in \check{H}^{q}\left(X, A_{2} ; R\right)$, então em $\check{H}^{p+q}\left(X, A_{1} \cup A_{2} ; R\right)$ temos

$$
u \smile v=(-1)^{p q} v \smile u .
$$

Finalizamos esta seção, observando que existe uma relação entre a cohomologia de Čech e a cohomologia de Alexander (Para detalhes, vide [49]) mediante um isomorfismo natural, como mostra a seguinte

ProposiçÃo 1.3.24. [49, Capítulo 6, Ex.3, pag.359] Para todo par topológico $(X, A)$ existe um isomorfismo natural

$$
\check{H}^{q}(X, A ; R) \cong \bar{H}^{q}(X, A ; R)
$$

onde $\bar{H}^{q}$ denota o funtor cohomologia de Alexander. 


\section{Grupos de transformações compactos}

A categoria na qual iremos desenvolver os conceitos e resultados deste trabalho é a categoria G-Top ${ }_{p c}^{2}$, que contém como objetos os $G$-pares paracompactos $(X, A)$, onde $G$ é um grupo compacto de Lie, e cujos morfismos $f:(X, A) \rightarrow(Y, B)$ são as aplicações de pares $G$-equivariantes, ou seja, pares paracompactos $(X, A)$ com uma determinada $G$-ação e aplicações $f:(X, A) \rightarrow(Y, B)$ que possuem a propriedade de comutar com a $G$-ação dada. Neste capítulo, as seções estão organizadas como segue: na Seção 2.1, apresentamos resultados básicos sobre grupos topológicos. Na Seção 2.2 introduzimos a teoria de ações de um grupo topológico $G$ sobre um espaço topológico $X$, definindo os conceitos de $G$-espaços e de aplicações $G$-equivariantes. Como um caso particular, na Subseção 2.2.1 consideramos o caso em que $G$ é um grupo compacto de Lie e $X$ é um espaço paracompacto, para assim definir os objetos e morfismos da categoria G-Top ${ }_{p c}^{2}$. Na Seção 2.3, apresentamos uma classe especial de fibrados, os chamados $G$-fibrados principais, onde $G$ é um grupo topológico. Tais fibrados são caracterizados pela propriedade de que a fibra do fibrado é o próprio grupo $G$, o qual age sobre si mesmo por translações. $G$-fibrados universais, espaços classificantes e aplicações classificantes são introduzidos, juntamente com a construção de Milnor, segundo a qual o espaço classificante $B G$ do $G$-fibrado universal é descrito como sendo o limite direto do quociente de joins do grupo $G$. Na Subseção 2.3.4 descrevemos a construção de Borel sobre os espaços classificantes de um grupo compacto de Lie. Tal construção é um procedimento para criar novos espaços e aplicações, através do $G$-fibrado universal, os quais serão a base da definição da cohomologia $G$-equivariante de Borel estudada no Capítulo 3. Embora este capítulo trate dos grupos de transformação compactos, o nosso objetivo aqui não é proporcionar um estudo sistemático da teoria das $G$-ações sobre espaços topológicos, mas apenas estabelecer uma linguagem básica para o estudo da teoria de índice de um $G$-espaço e o grau de uma aplicação $G$-equivariante. Exemplos importantes de $G$ espaços e $G$-ações serão detalhados nos Capítulos 3 e 4, no caso de grupos compactos de Lie. 


\subsection{Grupos topológicos}

Nesta seção, introduzimos um breve resumo do conceito de grupo topológico e apresentamos algumas de suas principais propriedades. Detalhes desses resultados podem ser encontrados em [8], [10], [11] e [40].

DEFINIÇÃo 2.1.1. Um grupo topológico é uma tripla $(G, *, \tau)$ onde $(G, *)$ é um grupo algébrico e $(G, \tau)$ é um espaço topológico de Hausdorff, cuja topologia $\tau$ é compatível com a operação $*$ do grupo, ou seja, as aplicações:

$$
\begin{aligned}
\mu:\left(G \times G, \tau_{\text {prod }}\right) & \rightarrow(G, \tau) \\
(g, h) & \mapsto \mu(g, h)=g * h \\
\iota:(G, \tau) & \rightarrow(G, \tau) \\
g & \mapsto \iota(g)=g^{-1},
\end{aligned}
$$

chamadas multiplicação e inversão, respectivamente, são contínuas, onde $\tau_{\text {prod }}$ denota a topologia produto sobre $G \times G$. O elemento neutro do grupo $(G, *)$ será denotado por $e$.

DEFINIÇÃo 2.1.2. Um grupo topológico $(G, *, \tau)$ é chamado compacto, se $(G, \tau)$ for um espaço topológico compacto.

OBSERVAÇÃo 2.1.3. Se $(G, *, \tau)$ for um grupo topológico, dado um subgrupo $H$ de $G$, então $\left(H, *, \tau_{H}\right)$ é um grupo topológico, com relação à topologia induzida de subespaço $\tau_{H}$. Se $H$ for um subespaço compacto, então $\left(H, *, \tau_{H}\right)$ é um grupo topológico compacto.

obSERVAÇÃo 2.1.4. A continuidade da aplicações $\mu$ e $\iota$, exigidas na Definição 2.1.1, são equivalentes às seguintes condições:

1. Dados $(g, h) \in G \times G$ e $W \in \tau, \operatorname{com} \mu(g, h)=g * h \in W$, existem $U, V \in \tau$ tais que $(g, h) \in U \times V$ e $\mu(U \times V)=U * V \subset W$, onde $U * V=\{g * h, g \in U$ e $h \in V\}$.

2. Dados $g \in G$ e $V \in \tau$, $\operatorname{com} \iota(g)=g^{-1} \in V$, existe $U \in \tau$ tal que $g \in U$ e $\mu(U)=$ $U^{-1} \subset V$, onde $U^{-1}=\left\{g^{-1}, g \in U\right\}$.

obSERVAÇÃo 2.1.5. Dados um grupo $(G, *)$ e um subgrupo $H$ de $G$, a coleção

$$
G / H=\{g * H ; g \in G\},
$$

das classes laterais à esquerda de $H$ em $G$, munida da operação

$$
(g * H) \cdot(h * H)=(a * b) * H,
$$

é um grupo se, e somente se, $H$ é um subgrupo normal de $G$, ou seja, $g * H * g^{-1}=H$, $\forall g \in G$. Neste caso, $G / H=\{g * H ; g \in G\}=\{H * g, g \in G\}$. Denotaremos a projeção natural $g \mapsto g * H$ por $\varphi: G \rightarrow G / H$. 
Proposição 2.1.6. Seja $(G, *, \tau)$ um grupo topológico. Então, são válidos os seguintes resultados:

1. Se $H$ for um subgrupo fechado de $(G, *, \tau)$, então o espaço quociente $G / H$, munido da topologia quociente induzida pela projeção canônica $\varphi: G \rightarrow G / H$, é um espaço de Hausdorffe $\varphi$ é continua e aberta (Vide [8, Proposição 1.4, Pg. 2]).

2. Se $H$ for um subgrupo fechado e normal de $(G, *, \tau)$, então $\left(G / H, \cdot, \tau_{\varphi}\right)$ é um grupo topológico (Vide [8, Proposição 1.5, Pg. 2]).

3. Se $G_{0}$ for a componente conexa de $G$, contendo o elemento identidade e $\in G$, então $G_{0}$ é um subgrupo fechado e normal de $G$ (Vide [8, Proposição 1.6, Pg. 3]).

4. As componentes conexas de G estão em correspondência um-a-um com as classes laterais de $G / G_{0}$ (Vide [40, Teorema 1.12, Pg.13]).

DEFIniÇão 2.1.7. Um grupo topológico $(G, *, \tau)$ é chamado um Grupo de Lie, se $G$ for uma $C^{\infty}$-variedade diferenciável tal que as operações multiplicação e inversão do grupo $(G, *)$, dadas na Definição 2.1.1 são aplicações de classe $C^{\infty}$.

PROPOSIÇÃo 2.1.8. Seja G um grupo de Lie.

1. Se $H \subset G$ for um subgrupo fechado, então $H$ é um grupo de Lie, com a estrutura diferenciável de uma subvariedade de G (Vide [40, Teorema 5.13, Pg.43]).

2. Se $G$ for um grupo compacto e se $G_{0}$ for a componente conexa de $G$ contendo o elemento identidade e $\in G$, então $G / G_{0}$ é um grupo finito (Vide [24, Pg.116]).

EXEMPLO 2.1.9. Qualquer grupo arbitrário $(G, *)$ é um grupo topológico com a topologia discreta, na qual todo subconjunto de $G$ é aberto. Em particular, se $G$ for finito, então $G$ é um grupo compacto.

EXEMPLO 2.1.10. Seja $\left\{\left(G_{\alpha}, *_{\alpha}, \tau_{\alpha}\right)\right\}_{\alpha \in J}$ uma família de grupos topológicos e consideremos o espaço produto $\left(\prod_{\alpha \in J} G_{\alpha}, \prod \tau_{\alpha}\right)$, onde $\prod \tau_{\alpha}$ denota a topologia produto. Então, as operações

$$
\begin{aligned}
\mu:\left(\prod_{\alpha \in J} G_{\alpha}\right) \times\left(\prod_{\alpha \in J} G_{\alpha}\right) & \rightarrow \prod_{\alpha \in J} G_{\alpha} \\
\left(\left(x_{\alpha}\right)_{\alpha},\left(y_{\alpha}\right)_{\alpha}\right) & \mapsto\left(x_{\alpha} *_{\alpha} y_{\alpha}\right)_{\alpha} \\
\iota: \prod_{\alpha \in J} G_{\alpha} & \rightarrow \prod_{\alpha \in J} G_{\alpha} \\
\left(x_{\alpha}\right)_{\alpha} & \mapsto\left(x_{\alpha}^{-1}\right)_{\alpha},
\end{aligned}
$$

são contínuas e, portanto, $\left(\prod_{\alpha \in J} G_{\alpha}, \prod \tau_{\alpha}\right)$ é um grupo topológico.

EXEMPLo 2.1.11. A esfera $S^{1}=\{z \in \mathbb{C}:|z|=1\} \cong \mathbb{R} / \mathbb{Z}$ é um subgrupo multiplicativo de $\mathbb{C}$ e o isomorfismo $\mathbb{R} / \mathbb{Z} \rightarrow S^{1}$ é induzido pela aplicação $t \mapsto e^{2 \pi i t}$. Desde que a multiplicação em $\mathbb{C}$ é associativa e os inversos existem, pois se $\|z\|=1$, então $z \bar{z}=\|z\|^{2}=1$, segue que $S^{1}$, munida da topologia induzida de $\mathbb{C}$, é um grupo compacto de Lie. 
EXEMPLO 2.1.12. O grupo $G L(n, \mathbb{R})$ das matrizes $n \times n$ inversíveis com entradas reais, é um grupo topológico, chamado o Grupo Linear Geral, quando considerado como um subespaço do $n^{2}$-dimensional espaço vetorial $M(n, \mathbb{R})$ de todas as matrizes $n \times n$. $G L_{n}(\mathbb{R})$ é um subespaço aberto de $M(n, \mathbb{R})$, desde que as matrizes inversíveis são aquelas cujo determinante é não nulo e, a função determinante:

$$
\begin{aligned}
\text { det } & : M(n, \mathbb{R}) \rightarrow \mathbb{R} \\
A & \mapsto \operatorname{det}(A) .
\end{aligned}
$$

é de classe $C^{\infty}$. Além disso, a multiplicação e a inversão de matrizes são aplicações de classe $C^{\infty}$. Portanto, $G L(n, \mathbb{R})$ é um grupo de Lie. Seus subgrupos clássicos:

$$
\begin{aligned}
S L(n, \mathbb{R}) & =\{A \in G L(n, \mathbb{R}) ; \operatorname{det}(A)=1\} \\
O(n) & =\left\{A \in G L(n, \mathbb{R}) ; A \cdot A^{t}=I\right\},
\end{aligned}
$$

são grupos topológicos, com a topologia induzida de $M(n, \mathbb{R})$, chamados, respectivamente, Grupo Especial Linear e Grupo Ortogonal, onde $A^{t}$ denota a matriz transposta de $A$ e $I$ denota a matriz identidade. Desde que ambos são subgrupos fechados de $G L(n, \mathbb{R})$ (Vide [40, Pgs.15 e 19]), segue da Proposição 2.1.8(1), que $S L(n, \mathbb{R})$ e $O(n)$ são grupos de Lie (Vide também [40, Corolário 5.14, Pg.43]). Mostra-se ainda que $O(n)$ é um grupo compacto (Vide [34, Pg.57]).

\section{$2.2 G$-espaços e aplicações $G$-equivariantes}

Os conceitos básicos sobre categorias, paracompacidade e join de espaços, citados aqui, podem ser encontrados nas seções A.2, A.3 e A.4 do Apêndice A. Para um grupo topológico fixado $G$, existe uma categoria ${ }^{1} G$-Top, cujos objetos são os espaços topológicos que admitem uma ação do grupo $G$, os chamados $G$-espaços, e cujos morfismos são as aplicações contínuas chamadas $G$-equivariantes, as quais comutam com a $G$-ação. A partir de agora, grupos topológicos $(G, *, \tau)$ e espaços topológicos $\left(X, \tau^{\prime}\right)$, serão denotados simplesmente por $G$ e $X$, respectivamente.

DEFINIÇÃo 2.2.1. Uma ação à esquerda de um grupo topológico $G$ sobre um espaço topológico $X$, também chamada uma $G$-ação à esquerda de $G$ sobre $X$, é uma função contínua

$$
\begin{aligned}
\rho: G \times X & \rightarrow X \\
(g, x) & \mapsto \rho(g, x) \stackrel{\text { notacăa }}{=} g x,
\end{aligned}
$$

onde $G \times X$ é munido da topologia produto, satisfazendo as seguintes condições:

(1) $g(h x)=(g * h) x, \forall x \in X$ e $\forall g, h \in G$.

(2) $e x=x, \forall x \in X$.

\footnotetext{
${ }^{1}$ Vide Definição A.2.1 e Exemplo A.2.3
} 
DEFINIÇÃO 2.2.2. Um G -espaço à esquerda, também chamado Grupo de Transformação, é um par $(X, \rho)$, consistindo de um espaço topológico de Hausdorff $X$ e uma $G$-ação à esquerda $\rho$ de um grupo topológico $G$ sobre $X$. Se $(X, \rho)$ for um $G$-espaço à esquerda, dizemos que o grupo $G$ age (ou atua) à esquerda sobre o espaço topológico $X$.

OBSERVAÇÃo 2.2.3. No caso particular em que o $G$-espaço $(X, \rho)$ é uma variedade topológica, dizemos que $X$ é uma $\boldsymbol{G}$-variedade topológica.

OBSERVAÇÃo 2.2.4. Também é possível definir uma $G$-ação à direita de um grupo topológico $G$ sobre um espaço topológico $X$ da seguinte forma: uma ação à direita de um grupo topológico $G$ sobre um espaço topológico $X$, chamada uma $G$-ação à direita de $G$ sobre $X$, é uma função contínua

$$
\begin{aligned}
\rho: \quad X \times G & \rightarrow X \\
(x, g) & \mapsto x g,
\end{aligned}
$$

satisfazendo as seguintes condições:

(1) $(x h) g=x(h * g), \forall x \in X$ e $\forall g, h \in G$.

(2) $x e=x, \forall x \in X$.

No contexto deste trabalho, de maneira geral, chamaremos uma $G$-ação à esquerda simplesmente de $G$-ação e um $G$-espaço à esquerda $(X, \rho)$, será chamado simplesmente um $G$-espaço. Quando estivermos nos referindo a ações à direita, usaremos então os termos $G$-ação à direita e $G$-espaço à direita.

EXEMPLo 2.2.5. Dados grupos topológicos $G_{1}$ e $G_{2}$, sejam $\left(X_{1}, \rho_{1}\right)$ um $G_{1}$-espaço e $\left(X_{2}, \rho_{2}\right)$ um $G_{2}$-espaço. Então, o produto $X_{1} \times X_{2}$ e o join ${ }^{2} X_{1} * X_{2}$ possuem estrutura de $\left(G_{1} \times G_{2}\right)^{3}$ espaço, onde

$$
\begin{aligned}
\rho:\left(G_{1} \times G_{2}\right) \times\left(X_{1} \times X_{2}\right) & \rightarrow X_{1} \times X_{2} \\
\left(\left(g_{1}, g_{2}\right),\left(x_{1}, x_{2}\right)\right) & \mapsto\left(g_{1} x_{1}, g_{2} x_{2}\right)
\end{aligned}
$$

é a $G_{1}$-ação sobre o espaço produto $X_{1} \times X_{2} \mathrm{e}$

$$
\begin{aligned}
\rho^{\prime}: \quad\left(G_{1} \times G_{2}\right) \times\left(X_{1} * X_{2}\right) & \rightarrow X_{1} * X_{2} \\
\left(\left(g_{1}, g_{2}\right), t_{1} x_{1}+t_{2} x_{2}\right) & \mapsto t_{1}\left(g_{1} x_{1}\right)+t_{2}\left(g_{2} x_{2}\right)
\end{aligned}
$$

é a $G_{2}$-ação sobre o join $X_{1} * X_{2}$. Esta ação é chamada ação produto.

DEFINIÇão 2.2.6. Dados um grupo topológico $G$ e um $G$-espaço $(X, \rho)$, para cada $g \in G$ fixado, podemos definir uma aplicação,

$$
\begin{aligned}
L_{g}: \quad X & \rightarrow X \\
x & \mapsto L_{g}(x)=g x
\end{aligned}
$$

chamada translação à esquerda por $g$.

\footnotetext{
${ }^{2}$ Vide Definição A.4.1

${ }^{3} \mathrm{O}$ Exemplo 2.1.10, garante que $G_{1} \times G_{2}$ é um grupo topológico.
} 
OBSERVAÇÃo 2.2.7. Observemos que, para cada a aplicação $g \in G, L_{g}$ é a restrição $\left.\rho\right|_{\{g\} \times X}$ : $\{g\} \times X \rightarrow X$, da ação contínua $\rho$. Assim, $L_{g}$ é contínua, para todo $g \in G$. Além disso, para cada $g \in G$ e para cada $x \in X$,

$$
\begin{aligned}
\left(L_{g} \circ L_{g^{-1}}\right)(x) & =L_{g}\left(L_{g^{-1}}(x)\right)=L_{g}\left(g^{-1} x\right) \\
& =g\left(g^{-1} x\right)=\left(g * g^{-1}\right) x=e x=x,
\end{aligned}
$$

ou seja, $\left(L_{g}\right)^{-1}=L_{g^{-1}}$ e, portanto, $L_{g}$ é um homeomorfismo, $\forall g \in G$.

obSERVAÇÃo 2.2.8. Dado um espaço topológico $X$, seja $\operatorname{Homeo}(X)$ o grupo (com a operação de composição) de todos os homeomorfismos de $X$ em $X$. Então, a aplicação $g \mapsto L_{g}$ define um homomorfismo

$$
\begin{aligned}
L: \quad G & \rightarrow \operatorname{Homeo}(X) \\
g & \mapsto L_{g}
\end{aligned}
$$

O kernel desse homomorfismo $L$ é chamado o "kernel da ação $\rho$ ", ou seja,

$$
\operatorname{Ker}(\rho)=\{g \in G ; g x=x, \forall x \in X\} .
$$

DEFINIÇÃo 2.2.9. Seja $(X, \rho)$ um $G$-espaço. O conjunto

$$
R=\{(x, g x) ; x \in X, g \in G\}
$$

é uma relação de equivalência sobre $X$. O conjunto das classes de equivalência determinado pela relação $R$ será denotado por $X / G$. A projeção natural $\pi: X \rightarrow X / G, x \mapsto[x]$, também chamada aplicação de órbitas, induz a topologia quociente sobre $X / G$, ou seja, $U \subset X / G$ é aberto se, e somente se, $\pi^{-1}(U)$ é aberto em $X$. O espaço quociente $X / G$ é chamado espaço de órbitas do $G$-espaço $(X, \rho)$.

A classe de equivalência de um ponto $x \in X$ é chamada a órbita de $x$ pela ação de $G$ e será denotada por $G x$, isto é,

$$
G x=[x]=\{g x \in X ; g \in G\} .
$$

observação 2.2.10. Seja $(X, \rho)$ um $G$-espaço, então a projeção natural $\pi: X \rightarrow X / G$, $x \mapsto G x$ é uma aplicação aberta. De fato, dado um aberto $U \subset X$, temos que

$$
\pi^{-1} \pi(U)=\bigcup_{g \in G} L_{g}(U)
$$

é aberto, desde que pela Observação 2.2.7, $L_{g}$ é um homeomorfismo. Segue da definição de topologia quociente que $\pi(U)$ é aberto.

OBSERVAÇÃo 2.2.11. Observemos que as órbitas $G x$ e $G y$ de quaisquer dois pontos $x, y \in$ $X$ ou são iguais ou são disjuntas.

DEFINIÇão 2.2.12. Dados um $G$-espaço $(X, \rho)$ e um ponto $x \in X$, o conjunto

$$
G_{x}=\{g \in G ; g x=x\}
$$

é um subgrupo topológico de $G$, chamado o subgrupo de isotropia do $G$-espaço $(X, \rho)$ em $x$. 
As ações de um grupo topológico $G$ sobre um espaço topológico $X$, podem ser classificadas de acordo a seguinte

DEFINIÇÃo 2.2.13. Seja $\rho: G \times X \rightarrow X$ uma $G$-ação de um grupo topológico $G$ sobre um espaço topológico $X$. Dizemos que

(1) $\rho$ é trivial, se $g x=x, \forall g \in G$ e $\forall x \in X$.

(2) $\rho$ é efetiva, se $g x=x, \forall x \in X \Rightarrow g=e$ ou, equivalentemente,

$$
\operatorname{Ker}(\rho)=\{g \in G ; g x=x, \forall x \in X\}=\{e\} .
$$

Em outras palavras, uma ação é efetiva, se cada elemento $g \neq e$ em $G$, move pelo menos um ponto de $X$.

(3) $\rho$ é livre, se $g x \neq x, \forall x \in X$ e $\forall g \neq e$, ou equivalentemente, se todos os subgrupos de isotropia são triviais, isto é,

$$
G_{x}=\{g \in G ; g x=x\}=\{e\}, \text { para todo } x \in X .
$$

Em outras palavras, uma ação é livre se cada elemento não trivial de $G$, move todo ponto de $X$.

(4) $\rho$ é transitiva, se $X / G$ consiste de um único ponto, ou seja, $X$ contém uma única órbita $G x=X$, para algum (e, portanto, para todo) $x \in X$.

OBSERVAÇÃo 2.2.14. Dada uma $G$-ação livre de um grupo topológico $G$ sobre um espaço topológico $X$, dizemos que $(X, \rho)$ é um $G$-espaço livre e que $G$ age livremente sobre $X$. A mesma terminologia é usada para os outros tipos de $G$-ações.

DEFINIÇÃo 2.2.15. Dizemos que um subconjunto $A$ de um $G$-espaço $(X, \rho)$ é $\boldsymbol{G}$-invariante pela ação $\rho$ de $G$ sobre $X$, se $g x \in A$, para todo $g \in G$ e para todo $x \in A$.

OBSERVAÇÃo 2.2.16. Dado um subconjunto $\boldsymbol{G}$-invariante $A$ de um $G$-espaço $(X, \rho)$, então $\rho$ induz uma $G$-ação contínua $G \times A \rightarrow A,(g, a) \mapsto g a$. Dessa forma, $A$ torna-se um $G$-espaço.

As aplicações continuas entre $G$-espaços que comutam com a $G$-ação são chamadas $G$ equivariantes. Formalmente, temos a seguinte

DEFINIÇão 2.2.17. Sejam $\left(X, \rho_{X}\right)$ e $\left(Y, \rho_{Y}\right) G$-espaços. Uma função contínua entre $G$ espaços $f: X \rightarrow Y$ é chamada uma aplicação $G$-equivariante ou uma $G$-aplicação se para todo $g \in G$ e para todo $x \in X$

$$
f(g x)=g f(x) .
$$

OBSERVAÇÃo 2.2.18. Para um grupo topológico fixado $G$, os $G$-espaços à esquerda e as aplicações $G$-equivariantes formam a categoria $G$-Top. Essa categoria possui produtos: dada uma família de $G$-espaços $\left\{\left(X_{j}, \rho_{j}\right) ; j \in J\right\}$, então o produto topológico $\prod_{j \in J} X_{j}$, pode ser munido de uma $G$-ação à esquerda

$$
\begin{aligned}
\rho: G \times \prod_{j \in J} X_{j} & \rightarrow \prod_{j \in J} X_{j} \\
\left(g,\left(x_{j}\right)\right) & \mapsto\left(g x_{j}\right),
\end{aligned}
$$


ou seja, $\left(\prod_{j \in J} X_{j}, \rho\right)$ é um produto na categoria $G$-Top.

Podemos ainda definir uma $G$-ação à esquerda sobre o join ${ }^{4} X=*_{j \in J} X_{j}$ da família de $G$-espaços $\left\{\left(X_{j}, \rho_{j}\right) ; j \in J\right\}$ como segue,

$$
\begin{aligned}
\rho: G \times *_{j \in J} X_{j} & \rightarrow *_{j \in J} X_{j} \\
\left(g, \sum t_{j} x_{j}\right) & \mapsto \sum t_{j} g x_{j} .
\end{aligned}
$$

Se os espaços $X_{j}$ forem $G$-espaços à direita, então

$$
\begin{aligned}
\rho: *_{j \in J} X_{j} \times G & \rightarrow *_{j \in J} X_{j} \\
\left(\sum t_{j} x_{j}, g\right) & \mapsto \sum t_{j} x_{j} g .
\end{aligned}
$$

Ambas definem ações contínuas de $G$ sobre $X=*_{j \in J} X_{j}$. A continuidade é verificada usando-se a propriedade universal da topologia do join. Portanto, $\left(*_{j \in J} X_{j}, \rho\right)$ é um $G$ espaço à esquerda e à direita, respectivamente.

DEFINIÇÃo 2.2.19. Uma ação $\rho$ definida pelas expressões dadas em (2.3), (2.4) ou (2.5), é chamada uma $G$-ação diagonal.

DEFINIÇão 2.2.20. Dados $G$-espaços $\left(X, \rho_{X}\right)$ e $\left(Y, \rho_{Y}\right)$, uma aplicação $G$-equivariante $f$ : $X \rightarrow Y$ induz, por passagem ao espaço de órbitas, uma aplicação

$$
\begin{aligned}
\bar{f}: \quad X / G & \rightarrow Y / G \\
G x=[x] & \mapsto G f(x)=[f(x)],
\end{aligned}
$$

chamada aplicação induzida nos espaços de órbitas, onde $G f(x)$ é a órbita do ponto $f(x) \in Y$.

No caso particular em que a $G$-ação sobre o espaço $X$ é determinada por um grupo compacto, o espaço de órbitas $X / G$ possui importantes propriedades, dadas na seguinte

Proposiç̧̃o 2.2.21. [8, Teorema 3.1, Pg. 38] Seja $(X, \rho)$ um G-espaço, onde G é um grupo compacto. Então,

(1) $X / G$ é Hausdorff.

(2) A projeção natural $\pi: X \rightarrow X / G$ é uma aplicação fechada.

(3) $\pi: X \rightarrow X / G$ é uma aplicação própria ${ }^{5}$.

(4) $X$ é compacto se, e somente se, $X / G$ for compacto.

COROLARIO 2.2.22. Se $(X, \rho)$ for um $G$-espaço paracompacto ${ }^{6}$, então o espaço de órbitas $X / G$ é paracompacto.

\footnotetext{
${ }^{4}$ Vide Definição A.4.6

${ }^{5}$ Uma função contínua $f: X \rightarrow Y$ é chamada uma aplicação própria se para todo compacto $K \subset Y$, $f^{-1}(K) \subset X$ é compacto.

${ }^{6}$ Vide Definição A.3.5
} 
Demonstração: Desde que a projeção $\pi: X \rightarrow X / G$ é uma aplicação contínua, fechada e sobrejetora, segue da Proposição A.3.12, que $X / G$ é paracompacto.

OBSERVAÇ̃̃o 2.2.23. Se $G$ for um grupo compacto de Lie e $M$ for uma variedade diferenciável $^{7}$, uma $G$-ação diferenciável é uma aplicação de classe $C^{\infty}$

$$
\begin{aligned}
\rho: G \times M & \rightarrow M \\
(g, m) & \mapsto g m,
\end{aligned}
$$

a qual é uma $G$-ação. Uma variedade diferenciável munida de uma $G$-ação diferenciável é chamada uma $G$-variedade diferenciável. Existe também o conceito de $G$-variedades localmente diferenciáveis (Vide [8, Capítulo IV]).

O resultado a seguir, foi provado por Bredon em [8, Capítulo IV, Teoremas 3.3 e 3.8], no caso em que $M$ é uma $G$-variedade localmente diferenciável, mas é válido, mais geralmente, para $G$-variedades topológicas (Vide Montgomery [41]).

PROPOSIÇÃo 2.2.24. Suponhamos que G seja um grupo compacto de Lie agindo livremente sobre uma variedade topológica compacta e conexa de dimensão finita $m$. Então, $M / G$ também é uma variedade topológica compacta conexa de dimensão $m-\operatorname{dim} G$.

\subsubsection{A categoria G-Top $p_{p c}^{2}: G$-pares paracompactos}

Nesta subseção, estaremos considerando $G$-ações na categoria G-Top ${ }_{p c}^{2}$, cujos objetos são $G$-pares paracompactos e cujos morfismos são as aplicações de pares $G$-equivariantes, onde $G$ é um grupo compacto de Lie. A partir de agora, um $G$-espaço $(X, \rho)$ será denotado simplesmente por $X$ e, quando for necessário, fixaremos a notação $\rho$ para a $G$-ação sobre $X$.

DEFINIÇão 2.2.25. Um par de espaços ${ }^{8}(X, A)$, no qual $X$ é um $G$-espaço e $A$ é um subespaço $G$-invariante, é chamado um $G$-par. Em particular, se $A=\emptyset$, escreveremos simplesmente $(X, \emptyset)=X$.

DEFINIÇão 2.2.26. Dados $G$-pares $(X, A)$ e $(Y, B)$, uma aplicação de pares ${ }^{9} f:(X, A) \rightarrow$ $(Y, B)$ é chamada uma aplicação de pares $G$-equivariante se $f: X \rightarrow Y$ for uma aplicação $G$-equivariante.

OBSERVAÇÃo 2.2.27. Dados $G$-pares $(X, A)$ e $(Y, B)$, uma aplicação de pares $G$-equivariante $f:(X, A) \rightarrow(Y, B)$ induz, por passagem ao quociente, uma aplicação de pares entre os espaços de órbitas

$$
\begin{aligned}
\bar{f}:(X / G, A / G) & \rightarrow(Y / G, B / G) \\
G x & \mapsto G f(x),
\end{aligned}
$$

chamada aplicação induzida nos espaços órbitas. Observemos que dado $G a \in A / G$, $\bar{f}(G a)=G f(a) \in B / G$, desde que $f(A) \subset B$.

\footnotetext{
${ }^{7}$ Por simplicidade de notação, o termo diferenciável aqui será usado para designar variedades diferenciáveis e aplicações diferenciáveis de classe $C^{\infty}$.

${ }^{8}$ Vide Definição A.1.1

${ }^{9}$ Vide Definição A.1.2
} 
DEFINIÇÃo 2.2.28. Dizemos que duas aplicações de pares $G$-equivariantes $f_{0}, f_{1}:(X, A) \rightarrow$ $(Y, B)$ são $G$-homotópicas, se existe uma aplicação de pares $G$-equivariante $F:(X \times I, A \times$ $I) \rightarrow(Y, B)$, chamada uma $G$-homotopia, tal que $F(x, 0)=f_{0}(x), F(x, 1)=f_{1}(x)$, onde $I=[0,1]$ possui a $G$-ação trivial e $X \times I, A \times I$ possuem a $G$-ação diagonal.

DEFINIÇÃo 2.2.29. Um par paracompacto $(X, A){ }^{10}$ que é também um $G$-par, onde $G$ é um grupo compacto de Lie, é chamado um $G$-par paracompacto.

OBSERVAÇão 2.2.30. Para um grupo compacto de Lie fixado $G$, a coleção de todos os $G$ pares paracompactos, juntamente com as aplicações de pares $G$-equivariantes, formam uma categoria, a qual será denotada por G-Top ${ }_{p c}^{2}$.

\subsection{G-fibrados universais}

Uma construção direta do $G$-fibrado universal, para um grupo topológico $G$ arbitrário, foi dada primeiramente por Milnor[39]. Tal construção utiliza a noção de join de espaços topológicos ${ }^{11}$. A construção de Milnor do espaço classificante $B G$ do $G$-fibrado universal $\omega_{G}=\left(E G, B G, p_{G}, G, G\right)$, é descrita como sendo o limite direto do quociente de joins do grupo $G$.

\subsubsection{G-fibrados principais}

Nosso objetivo nesta seção é apresentar os conceitos e as principais propriedades de uma classe especial de fibrados, os chamados $G$-fibrados principais, onde $(G, \cdot, \tau)$ é um grupo topológico. Tais fibrados são caracterizados pela propriedade de que a fibra do fibrado é o próprio grupo $G$, o qual age sobre si mesmo por translações. Estaremos adotando aqui a notação multiplicativa para a operação do grupo $G$. Os resultados apresentados aqui são baseados nas referências [10], [30] e [50].

DEFINIÇÃo 2.3.1. Dado um grupo topológico $G$, um $G$-fibrado principal à direita consiste de uma $G$-ação livre à direita

$$
\begin{aligned}
\rho: \quad E \times G & \rightarrow E \\
(x, g) & \mapsto x g
\end{aligned}
$$

e uma função contínua e sobrejetora $p: E \rightarrow B$, satisfazendo as seguintes condições:

(1) Para cada $x \in E$ e $g \in G, p(x g)=p(x)$.

(2) Para cada $b \in B$, existe um aberto $V$ contendo $b$ em $B$ e um $G$-homeomorfismo $\varphi$ : $p^{-1}(V) \rightarrow V \times G$ tal que o seguinte diagrama

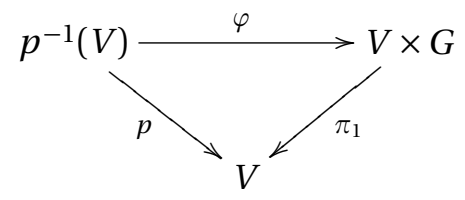

\footnotetext{
${ }^{10}$ Vide Definição A.3.14

${ }^{11}$ Vide Definição A.4.6
} 
é comutativo, onde $\pi_{1}: V \times G \rightarrow V$ é a projeção na primeira coordenada. $E$ é chamado espaço total, $B$ é chamado espaço base, $p: E \rightarrow B$ é chamada projeção, $G$ é a fibra do fibrado e $\varphi$ é chamado uma trivialização de $p$ sobre $V$, com fibra típica $G$. Aqui, $G$ age à direita sobre $V \times G$ via a ação

$$
\begin{aligned}
\rho:(V \times G) \times G & \rightarrow V \times G \\
((\nu, h), g) & \mapsto(\nu, h g) .
\end{aligned}
$$

Um fibrado é chamado trivial se existe uma trivialização sobre $B$.

OBSERVAÇÃO 2.3.2. Usaremos a notação $\xi=(E, p, B, G, G)$ para representar um $G$-fibrado principal com espaço total $E$, espaço base $B$, projeção $p: E \rightarrow B$ e grupo $G$.

EXEMPlo 2.3.3. Dados um espaço topológico $B$ e um grupo $G$, consideremos a $G$-ação trivial sobre $B$ e a $G$-ação diagonal à direita sobre $B \times G$ dada por

$$
\begin{aligned}
(B \times G) \times G & \rightarrow B \times G \\
((b, h), g) & \mapsto(b, h g) .
\end{aligned}
$$

Então, $\xi=\left(B \times G, p_{1}, B, G, G\right)$ é um $G$-fibrado principal, chamado G-fibrado produto (Vide [30, Capítulo 3, Exemplo 2.3]).

EXEMPLO 2.3.4. Seja $\xi=(E, p, B, G, G)$ um $G$-fibrado principal. Para cada espaço topológico $B^{*}$ e para cada função contínua $f: B^{*} \rightarrow B$, o fibrado induzido de $\xi$ por $f$, denotado por $f^{*}(\xi)$, tem como espaço base $B^{*}$ e seu espaço total é definido como sendo o subespaço

$$
f^{*}(E)=\left\{\left(b^{*}, x\right) \in B^{*} \times E ; f\left(b^{*}\right)=p(x)\right\},
$$

chamado o pull-back de $\xi$ por $f$. A projeção é dada pela aplicação

$$
p^{*}: f^{*}(E) \rightarrow B^{*}, \quad p^{*}\left(b^{*}, x\right)=b^{*} .
$$

Temos que $f^{*}(\xi)=\left(f^{*}(E), p^{*}, B^{*}, G, G\right)$ é um $G$-fibrado principal, chamado $G$-fibrado induzido de $\xi$ por $f$ (Vide [30, Capítulo 4, Proposição 4.1].

No contexto deste trabalho, o exemplo mais importante de $G$-fibrado principal é dado pela aplicação de órbitas $\pi: X \rightarrow X / G$, no caso em que $X$ é um espaço paracompacto e $G$ é um grupo compacto de Lie que age livremente sobre $X$. Nesse caso, temos o seguinte teorema fundamental

TEOREMA 2.3.5. Seja $X$ um G-espaço paracompacto, onde G é um grupo compacto de Lie agindo livremente sobre $X$. Então, $(X, \pi, X / G, G, G)$ é um $G$-fibrado principal, onde $\pi$ : $X \rightarrow X / G$ é a aplicação de órbitas.

obSERVAÇÃo 2.3.6. O teorema 2.3.5 foi provado por Bredon [8, Capítulo II, Teorema 5.8] no caso em que $X$ é um espaço completamente regular ${ }^{12}$. Desde que todo espaço paracompacto é normal ${ }^{13}$ e, pelo Lema de Urysohn, todo espaço normal é um espaço completamente regular, segue o resultado. Observemos ainda que, nas hipóteses do Teorema 2.3.5, segue do Corolário 2.2.22, que o espaço de órbitas $X / G$ é paracompacto.

\footnotetext{
${ }^{12} \mathrm{Um}$ espaço de Hausdorff $X$ é completamente regular se dados $x \in X$ e um fechado $A \subset X$ que não contém $x$, existe uma função contínua $f: X \rightarrow[0,1]$ tal que $f(x)=1$ e $f(a)=0, \forall a \in A$.

${ }^{13}$ Vide Proposição A.3.10
} 
DEFINIÇÃo 2.3.7. Sejam $\xi=(E, p, B, G)$ e $\eta=\left(E^{\prime}, p^{\prime}, B, G\right)$ dois $G$-fibrados principais sobre o mesmo espaço base $B$. Um $\boldsymbol{B}$-morfismo principal $u: \xi \rightarrow \eta$ é uma aplicação $G$-equivariante $u: E \rightarrow E^{\prime}$ tal que $p=p^{\prime} \circ u$, ou seja, o seguinte diagrama é comutativo.

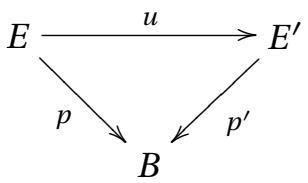

Um morfismo $u: \xi \rightarrow \eta$ entre dois $G$-fibrados principais sobre $B$ é chamado um $B$ isomorfismo, se $u: E \rightarrow E^{\prime}$ for um homeomorfismo.

\subsubsection{Espaços classificantes}

O objetivo desta seção é apresentar a construção do espaço classificante segundo Milnor[39]. Dado um grupo compacto de Lie, existe um espaço $B G$, chamado espaço classificante, e um $G$-fibrado universal $E G \rightarrow B G$ tal que para qualquer espaço paracompacto $B$, a construção "pulback"induz uma bijeção entre o conjunto $[B, B G]$ das classes de homotopia de aplicações de $B$ em $B G$ e a classe dos isomorfismos de $G$-fibrados principais sobre $B$. A referência clássica para este tópico é [30].

Apresentamos inicialmente o seguinte resultado fundamental para a construção de um $G$-fibrado universal.

LEMA 2.3.8. [30, Capítulo IV, Teorema 9.9] Seja $\xi=(E, p, B, G, G)$ um $G$-fibrado principal sobre um espaço paracompacto $B$ e suponhamos que as aplicações $u, v: B^{*} \rightarrow B$ sejam homotópicas. Então, os $G$-fibrados induzidos $u^{*}(\xi)$ e $v^{*}(\xi)$ são $B^{*}$-isomorfos.

Seja $B$ um espaço paracompacto. Definimos uma relação de equivalência como segue: dados dois $G$-fibrados principais $\xi$ e $\eta$ sobre $B$,

$$
\xi \sim \eta \quad \text { se, e somente se, } \quad \xi \text { é } B \text {-isomorfo a } \eta \text {. }
$$

A classe de equivalência de isomorfismos do $G$-fibrado principal $\xi$ sobre $B$ será denotada por $\{\xi\}$. O conjunto de todas as classes de equivalência de isomorfismos de $G$-fibrados principais sobre $B$ será denotado por $K_{G}(B)$.

Seja $\omega=\left(E_{0}, p_{0}, B_{0}, G, G\right)$ um $G$-fibrado principal sobre um espaço paracompacto $B_{0}$. Para cada espaço paracompacto $B$, definimos uma função:

$$
\phi_{\omega}(B):\left[B, B_{0}\right] \rightarrow K_{G}(B), \quad \phi_{\omega}(B)([u])=\left\{u^{*}(\omega),\right\}
$$

onde $u^{*}(\omega)$ é o $G$-fibrado induzido de $\omega$ por $u$ e $\left[B, B_{0}\right]$ denota o conjunto de todas as classes de homotopia $B \rightarrow B_{0}$. Esta função está bem definida, desde que se $[u]=[v]$, então $u, v: B \rightarrow B_{0}$ são homotópicas e segue do Lema 2.3.8 que $u^{*}(\omega)$ e $v^{*}(\omega)$ são $B$ isomorfos. Portanto, $\left\{u^{*}(\omega)\right\}=\left\{\nu^{*}(\omega)\right\}$.

DEFINIÇÃo 2.3.9. Um $G$-fibrado principal $\omega=\left(E_{0}, p_{0}, B_{0}, G, G\right)$ sobre o espaço paracompacto $B_{0}$ é chamado um $G$-fibrado universal se, para cada espaço paracompacto $B$, a função $\phi_{\omega}(B):\left[X, B_{0}\right] \rightarrow K_{G}(B)$ for uma bijeção. O espaço topológico $B_{0}$ é chamado um espaço classificante do grupo $G$ e usaremos as notações $E_{0}=E G, B_{0}=B G, p_{0}=p_{G} \mathrm{e}$ $\omega=\omega_{G}$. 
Temos assim o seguinte teorema de classificação de $G$-fibrados principais, no caso paracompacto

teorema 2.3.10. Teorema da Classificação[12, Capítulo 14, Teorema 14.4.1] Associamos a cada classe de isomorfismo de $G$-fibrados principais sobre um espaço paracompacto $B$ a classe de homotopia de uma aplicação classificante, obtendo uma bem definida bijeção $K_{G}(B) \cong[B, B G]$.

Seja $\omega_{G}=\left(E G, p_{G}, B G, G, G\right)$ um $G$-fibrado universal. Então, são válidos os seguintes resultados

PRoposição 2.3.11. Para cada $G$-fibrado principal $\xi=(E, p, B, G, G)$ sobre um espaço paracompacto $B$, existe uma aplicação continua $q: B \rightarrow B G$ tal que $\xi$ e o G-fibrado induzido $q^{*}\left(\omega_{G}\right)$ são $G$-fibrados principais $B$-isomorfos. A aplicação q $: B \rightarrow B G$ échamada uma aplicação classificante para o $G$-fibrado principal $\xi=(E, p, B, G, G)$.

Demonstração: Como $\phi_{\omega_{G}}(B)$ é bijetiva, então em particular é sobrejetiva. Logo dado um $G$-fibrado principal $\xi$ sobre um espaço paracompacto $B$, existe uma aplicação $q$ : $B \rightarrow B G$ tal que $\{\xi\}=\phi_{\omega_{G}}(B)([q])=\left\{q^{*}\left(\omega_{G}\right)\right\}$ então $\xi$ e $q^{*}\left(\omega_{G}\right)$ são $B$-isomorfos.

Proposição 2.3.12. Sejam $u, v: B \rightarrow B G$ duas aplicações continuas tais que $u^{*}\left(\omega_{G}\right) e$ $\nu^{*}\left(\omega_{G}\right)$ são $G$-fibrados principais B-isomorfos. Então u e u são homotópicas.

Demonstração: Como $\phi_{\omega_{G}}(B)$ é bijetiva, então em particular é injetiva. Logo se dadas duas aplicações $u, v: X \rightarrow B_{0}$ tais que $u^{*}\left(\omega_{G}\right)$ e $v^{*}\left(\omega_{G}\right)$ são $B$-isomorfos então

$$
\phi_{\omega_{G}}(B)([u])=\left\{u^{*}\left(\omega_{G}\right)\right\}=\left\{v^{*}\left(\omega_{G}\right)\right\}=\phi_{\omega_{G}}(B)([v])
$$

Se segue da injetividade de $\phi_{\omega_{G}}(B)$ que $[u]=[v]$. Portanto $u_{0}$ e $u_{1}$ são homotópicos.

Do teorema 2.3.5, temos que a aplicação de órbitas determina um $G$-fibrado principal, no caso em que $G$ é um grupo compacto de Lie e o espaço total desse fibrado é paracompacto. Além disso, nesse caso o espaço base é paracompacto. Assim, usando este fibrado, obtemos o seguinte teorema de grande importância para nosso trabalho:

TEOREMA 2.3.13. Sejam $X$ e $Y$ espaços paracompactos com ação livre de um grupo de compacto de Lie $G$. Denotemos por $\xi_{X} e \xi_{Y}$ os $G$-fibrados principais $(X, \pi, X / G, G, G) e$ $(Y, \pi, Y / G, G, G)$, respectivamente, com $X / G$ e $Y / G$ paracompactos. Seja $q_{X}: X / G \rightarrow B G$ uma aplicação classificante para $\xi_{X}$ e suponhamos que $f: X \rightarrow Y$ seja uma aplicação $G$-equivariante. Se $q_{Y}: Y / G \rightarrow B G$ for uma aplicação classificante para $\xi_{Y}$, então a aplicação $q_{Y} \circ \bar{f}: X / G \rightarrow B G$ também classifica o $G$-fibrado principal $\xi_{X}$. Em outras palavras, o seguinte diagrama

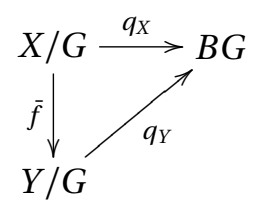

é homotopicamente comutativo, onde $\bar{f}: X / G \rightarrow Y / G$ é a induzida por $f$ entre os espaços de órbitas. 
Demonstração: Desde que $q_{X}: X / G \rightarrow B G$ é uma aplicação classificante para $\xi_{X}$, segue da proposição 2.3.11 que $\xi_{X}$ e $q_{X}^{*}\left(\omega_{G}\right)$ são $(X / G)$-isomorfos e, analogamente, $\xi_{Y}$ e $q_{Y}^{*}\left(\omega_{G}\right)$ são $(Y / G)$-isomorfos. Obtemos assim os seguintes diagramas comutativos
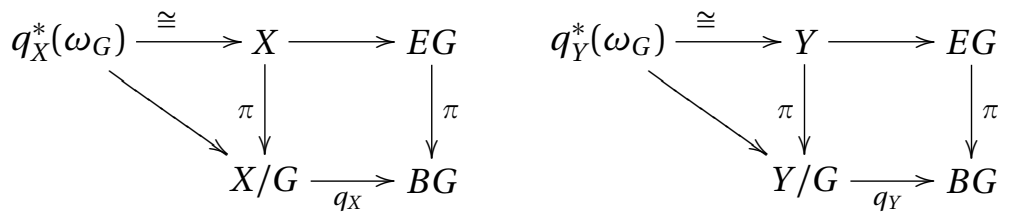

Desde que $f: X \rightarrow Y$ é uma aplicação $G$-equivariante, temos de [30, Capítulo 4, Teorema 4.2], que $\xi_{X}$ e o $G$-fibrado induzido $\bar{f}^{*}\left(\xi_{Y}\right)$ são $G$-fibrados principais $(X / G)$-isomorfos. Por outro lado, desde que $\xi_{Y}$ é isomorfo a $q_{Y}^{*}\left(\omega_{G}\right)$, segue de [30, Capítulo $4, \S 10$, que $\bar{f}^{*}\left(\xi_{Y}\right)$ é isomorfo a $\bar{f}^{*}\left(q_{Y}^{*}\left(\omega_{G}\right)\right)$.

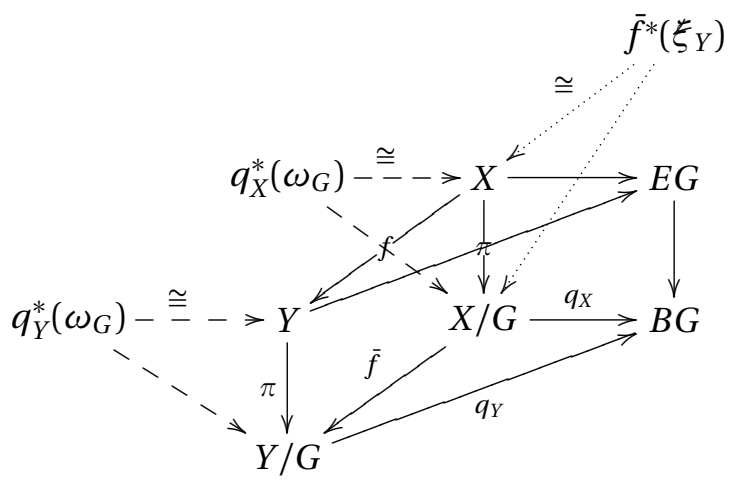

Alem disso, temos de [30, Capítulo 2, Proposição 5.7], que $\bar{f}^{*}\left(q_{Y}^{*}\left(\omega_{G}\right)\right)$ e $\left(q_{Y} \circ \bar{f}\right)^{*}\left(\omega_{G}\right)$ são $G$-fibrados principais $(X / G)$-isomorfos e, portanto $\xi_{X}, \mathrm{e}\left(q_{Y} \circ \bar{f}\right)^{*}\left(\omega_{G}\right)$ são $G$-fibrados principais $(X / G)$-isomorfos, o que implica da Proposição 2.3.11, que $q_{Y} \circ \bar{f}$ é uma aplicação classificante para o $G$-fibrado principal $\xi_{X}$. Segue da Proposição 2.3.12, que $q_{Y} \circ \bar{f}$ é homotópica a $q_{X}$.

\subsubsection{A construção de Milnor}

A construção de Milnor do espaço classificante $B G$ do $G$-fibrado universal $\omega_{G}=\left(E G, B G, p_{G}, G, G\right)$ é descrita como sendo o limite direto do quociente de joins do grupo $G$. Embora essa construção seja a mais geral possível, no contexto do nosso trabalho, estaremos considerando $G$ um grupo compacto de Lie.

Consideremos o join de $(n+1)$-cópias de $G$

$$
E G(n)=G * \cdots * G,
$$

munido da topologia de Milnor ${ }^{14}$. Assim, temos uma sequência de inclusões

$$
G \stackrel{l}{\hookrightarrow} \cdots \stackrel{l}{\hookrightarrow} E G(n) \stackrel{l}{\hookrightarrow} E G(n+1) \stackrel{l}{\hookrightarrow} \cdots
$$

${ }^{14}$ Vide Definição A.4.6 
Dessa forma, a coleção $\left\{E G(n), f^{m n}=\imath, \mathbb{Z}^{+}, n \leq m\right\}$ é um sistema direto de espaços topológicos ${ }^{15}$, indexado no conjunto dirigido $\mathbb{Z}^{+}$. Segue da Proposição 1.1.18 que

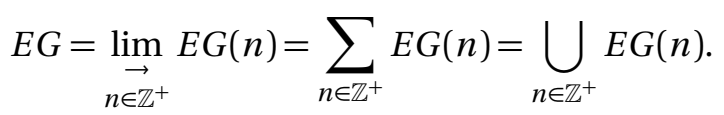

Milnor define uma $G$-ação à direita sobre cada $E G(n)$

$$
\begin{array}{cl}
E G(n) \times G & \rightarrow E G(n) \\
\left(t_{0} g_{0}+\ldots+t_{n} g_{n}, g\right) & \mapsto t_{0}\left(g_{0} g\right)+\ldots+t_{n}\left(g_{n} g\right),
\end{array}
$$

a qual induz uma $G$-ação à direita sobre o espaço $E G$, como foi definido em (2.5). Mostrase que essa ação é livre. O espaço de órbitas

$$
E G / G
$$

determinado pela $G$-ação livre sobre $E G$ será denotado por $B G$ e

$$
p_{G}: E G \rightarrow E G / G=B G
$$

denotará a aplicação de órbitas.

obSERVAÇÃo 2.3.14. Desde que $G$ é compacto Hausdorff, temos da Proposição A.4.7, que cada $E G(n)$ é compacto Hausdorff e segue da Proposição A.3.7 que cada $E G(n)$ é paracompacto. Como a união disjunta de espaços pacompactos é um espaço paracompacto (Vide Observação 1.1.19), temos que $E G$ é paracompacto, logo $E G$ é normal ${ }^{16} \mathrm{e}$, consequentemente, regular. Por outro lado, sendo uma reunião enumerável de espaços compactos, temos da Definição A.3.16, que $E G$ é $\sigma$-compacto e assim, concluimos que $E G$ é um espaço regular e $\sigma$-compacto. Portanto, segue da Observação A.3.17, que se $X$ for um espaço paracompacto, então o produto cartesiano $E G \times X$, munido da topologia produto, será um espaço paracompacto.

observaÇão 2.3.15. Segue do Teorema 2.3.5 e das Observações 2.3.14 e 2.3.6, que $\left(E G, p_{G}, B G, G, G\right)$ é um $G$-fibrado principal com espaço base $B G$ paracompacto.

Enunciamos a seguir o Teorema de Milnor, no caso particular em que $G$ é um grupo compacto de Lie, cuja demonstração no caso geral pode ser encontrada em [12], Capítulo 14, Teorema 14.4.2 e [39].

TEOREMA 2.3.16. Dado um grupo compacto de Lie G, existe um G-fibrado universal $\omega_{G}=\left(E G, p_{G}, B G, G, G\right)$, com $E G$ contrátil e $B G=E G / G$.

obSERVAÇÃo 2.3.17. Sejam $G_{1}$ e $G_{2}$ grupos compactos de Lie. Então, temos as seguintes identificações

$$
\left(G_{1} * \cdots * G_{1}\right) \times\left(G_{2} * \cdots * G_{2}\right) \approx\left(G_{1} \times G_{2}\right) * \cdots *\left(G_{1} \times G_{2}\right)
$$

as quais nos leva às seguintes identificações:

$$
E G_{1} \times E G_{2} \approx E\left(G_{1} \times G_{2}\right) \quad B G_{1} \times B G_{2} \approx B\left(G_{1} \times G_{2}\right)
$$

${ }^{15}$ Vide Seção 1.1.2

${ }^{16}$ Vide Proposição A.3.10 
EXEMPLO 2.3.18. Seja $G=\mathbb{Z}_{2}$, então o espaço $E G(n)$ é o join

$$
\mathbb{Z}_{2} * \cdots * \mathbb{Z}_{2}
$$

de $(n+1)$-cópias de $\mathbb{Z}_{2}$, o qual é homeomorfo à $n$-esfera $S^{n}$ e a ação de $\mathbb{Z}_{2}$ sobre $E G(n)=$ $S^{n}$ é definida pela identidade e pela aplicação antipodal. O espaço $B G(n)$ é $\mathbb{R} P^{n}$. As inclusões $E G(n) \subset E G(n+1)$ e $B G(n) \subset B G(n+1)$ são as inclusões naturais $S^{n} \subset S^{n+1}$ e $\mathbb{R} P^{n} \subset \mathbb{R} P^{n+1}$. O espaço $E G$ é $S^{\infty}$ e $B G$ é $\mathbb{R} P^{\infty}$. O $\mathbb{Z}_{2}$-fibrado $\left(S^{n+1}, p, \mathbb{R} P^{n+1}\right)$ é um $\mathbb{Z}_{2}$-fibrado universal para dimensões menores ou iguais a $n$.

EXEMPLo 2.3.19. Seja $G=S^{1}$, então o espaço $E G(n)$ é o join

$$
S^{1} * \cdots * S^{1}
$$

de $(n+1)$-cópias de $S^{1}$, o qual é homeomorfo à esfera $S^{2 n+1}$ e a ação de $S^{1}$ sobre $E G(n)=$ $S^{2 n+1}$ é dada pela relação

$$
e^{i \theta}\left(z_{0}, z_{1}, \ldots, z_{n}\right)=\left(e^{i \theta} z_{0}, e^{i \theta} z_{1}, \ldots, e^{i \theta} z_{n}\right)
$$

para cada $e^{i \theta} \in S^{1}$. O espaço $B G(n)$ é o $n$-dimensional espaço projetivo complexo $\mathbb{C} P^{n}$. As inclusões $E G(n) \subset E G(n+1)$ e $B G(n) \subset B G(n+1)$ são as inclusões naturais $S^{2 n+1} \subset$ $S^{2 n+3}$ e $\mathbb{C} P^{n} \subset \mathbb{C} P^{n+1}$. O espaço $E G$ é $S^{\infty}$ e $B G$ é $\mathbb{C} P^{\infty}$. O $S^{1}$-fibrado $\left(S^{2 n+1}, p, \mathbb{C} P^{n}\right)$ é um $S^{1}$-fibrado universal para dimensões menores ou iguais a $2 n$.

OBSERVAÇão 2.3.20. Será provado na Proposição 3.4.1 que as ações dadas nos Exemplos 2.3.18 e 2.3.19 são livres.

\subsubsection{A Construção de Borel}

Nesta seção, descrevemos a construção de Borel sobre os espaços classificantes de um grupo compacto de Lie. Tal construção é um procedimento para criar novos espaços e aplicações, através do $G$-fibrado universal, os quais serão a base da definição da cohomologia $G$-equivariante de Borel estudada no Capítulo 3. Os resultados apresentados aqui podem ser econtrados nas referências [3], [5] e [10].

Seja $G$ um grupo compacto de Lie e consideremos o $G$-fibrado universal

$$
\left(E G, p_{G}, B G, G, G\right)
$$

com $E G$ contrátil e $B G=E G / G$, apresentado na Subseção 2.3.3.

Dado um $G$-espaço $X$, a ação diagonal ${ }^{17}$ sobre o produto $E G \times X$, é uma $G$-ação livre. Com efeito, desde que a $G$-ação diagonal sobre $E G$ é livre, temos que $g m \neq m, \forall m \in E G$ $\mathrm{e} \forall g \neq e$. Assim,

$$
g(m, x)=(g m, g x) \neq(m, x), \quad \forall \quad(m, x) \in E G \times X \quad \text { e } \quad \forall g \neq e .
$$

${ }^{17}$ Vide Definição 2.2.19 
DEFINIÇÃo 2.3.21. Dado um $G$-espaço $X$, definimos o espaço de Borel, o qual será denotado por

$$
X_{G}=E G \times_{G} X
$$

como sendo o espaço de órbitas do produto $E G \times X$ com relação à $G$-ação diagonal livre, ou seja,

$$
X_{G}=E G \times_{G} X=\frac{E G \times X}{G}
$$

As órbitas $G(m, x)$, serão denotadas por $[m, x]$.

OBSERVAÇÃo 2.3.22. Seja $X$ um $G$-espaço paracompacto, onde $G$ é um grupo compacto de Lie. Segue da Observação 2.3.14 que $E G \times X$ é paracompacto e, desde que esse espaço admite ação livre de $G$, segue do Teorema 2.3.5 que

$$
\left(E G \times X, \pi, X_{G}, G, G\right)
$$

é um $G$-fibrado principal, com espaço base $X_{G}$ paracompacto (Vide Observação 2.3.6). Segue da Proposição 2.3.11, que existe uma aplicação classificante para o $G$-fibrado prin$\operatorname{cipal}\left(E G \times X, \pi, X_{G}, G, G\right)$ :

$$
q_{X}: X_{G} \rightarrow B G
$$

Por outro lado, a aplicação identidade Id $:$ BG $\rightarrow$ BG classifica o $G$-fibrado principal $\omega_{G}=$ $\left(E G, p_{G}, B G, G, G\right)$. Con efeito, provemos que o $G$-fibrado induzido $\operatorname{Id}^{*}\left(\omega_{G}\right)$ e $\omega_{G}$ são $B G$-isomorfos. De fato $\operatorname{Id}^{*}(E G)=\{(G m, m) \in B G \times E G: m \in E G\}, p^{*}(G m, m)=G m$ e a aplicação

$$
\chi: \operatorname{Id}^{*}(E G) \rightarrow E G \quad \chi(G m, m)=m
$$

é um homeomorfismo. Além disso, $p_{G} \circ \chi=p^{*}$.

Desde que projeção na primeira coordenada

$$
\pi_{1}: E G \times X \rightarrow E G
$$

é uma aplicação $G$-equivariante e a aplicação identidade Id : BG $\rightarrow$ BG classifica o $G$ fibrado principal $(E G, \pi, B G, G, G)$, segue do Teorema 2.3.13 que a aplicação induzida nos espaços órbitas faz o seguinte diagrama, comutativo homotopicamente

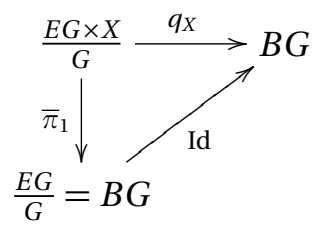

ou seja, $\bar{\pi}_{1}=\operatorname{Id} \circ \bar{\pi}_{1}, q_{X}: X_{G} \rightarrow B G$ são aplicações homotópicas. Obtemos assim, um diagrama comutativo

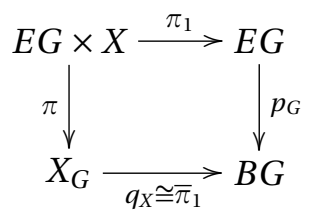

onde as funções verticais $\pi$ e $p_{G}$ são as respectivas aplicações de órbitas. 
Seja $(X, A)$ um $G$-par. Então, temos que

$E G \times A \subset E G \times X$, o que implica que $A_{G}=E G \times_{G} A=\frac{E G \times A}{G} \subset X_{G}$.

DEFINIÇ̃̃o 2.3.23. O par $\left(X_{G}, A_{G}\right)$ construído acima é chamado um par de Borel.

obServação 2.3.24. Dados $G$-espaços $A$ e $B$, então $(A \cup B)_{G}=A_{G} \cup B_{G}$.

Seja $f: X \rightarrow Y$ uma aplicação $G$-equivariante. Então, a aplicação

$$
\begin{aligned}
\operatorname{Id}_{E G} \times f: \quad E G \times X & \rightarrow E G \times Y \\
(m, x) & \mapsto(m, f(x))
\end{aligned}
$$

é uma aplicação $G$-equivariante, desde que

$$
\begin{aligned}
\left(\operatorname{Id}_{E G} \times f\right)(g(m, x)) & =\left(\operatorname{Id}_{E G} \times f\right)(g m, g x)=(g m, f(g x)) \\
& =(g m, g f(x))=g\left(\left(\operatorname{Id}_{E G} \times f\right)(m, x)\right)
\end{aligned}
$$

Logo, a passagem aos espaços órbitas nos leva à seguinte

DEFINIÇÃo 2.3.25. Seja $f: X \rightarrow Y$ uma aplicação $G$-equivariante. Definimos a aplicação de Borel como sendo a aplicação contínua

$$
f_{G}: \overline{I d_{E G} \times f}: X_{G} \rightarrow Y_{G}, \quad[m, x] \mapsto[m, f(x)]
$$

Mais geralmente, se $f:(X, A) \rightarrow(Y, B)$ é uma aplicação de pares $G$-equivariante, então $f_{G}\left(A_{G}\right) \subset B_{G}$. A aplicação de pares

$$
f_{G}:\left(X_{G}, A_{G}\right) \rightarrow\left(Y_{G}, B_{G}\right)
$$

é chamada aplicação de pares de Borel.

Uma consequência do teorema 2.3.13 para a construção de Borel é o seguinte

TEOREMA 2.3.26. Seja $f: X \rightarrow Y$ uma aplicação $G$-equivariante, onde $X$ e $Y$ são paracompactos. Então, o seguinte diagrama

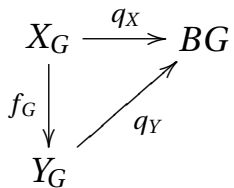

é homotopicamente comutativo, ou seja, $q_{X} \cong q_{Y} \circ f_{G}$.

Demonstração: Sejam $q_{X}$ e $q_{Y}$ as aplicações classificantes para os $G$-fibrados principais $\left(E G \times X, \pi, X_{G}, G, G\right)$ e $\left(E G \times Y, \pi, Y_{G}, G, G\right)$. Desde que

$$
\operatorname{Id}_{E G} \times f: E G \times X \rightarrow E G \times Y
$$

é uma aplicação $G$-equivariante entre os $G$-espaços paracompactos $E G \times X$ e $E G \times Y$, segue do teorema 2.3.13 que o Diagrama 2.12 é homotopicamente comutativo. 
PROPOSIÇ̃̃o 2.3.27. Se $f, f^{\prime}:(X, A) \rightarrow(Y, B)$ são G-homotópicas no sentido da Definição 2.2.28, então $f_{G}$ é homotópica a $f_{G}^{\prime}$.

Demonstração: Consideremos o seguinte diagrama comutativo

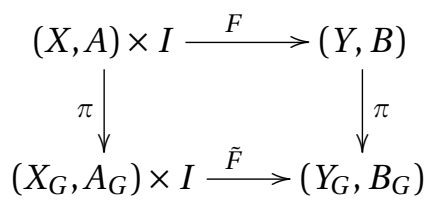

onde $F$ é uma $G$-homotopia entre $f$ e $f^{\prime}$, ou seja, $F$ é uma aplicação de pares $G$-equivariante satisfazendo as condições $F(x, 0)=f(x)$ e $F(x, 1)=f^{\prime}(x)$. Obtemos assim, uma aplicação contínua bem definida

$$
\begin{aligned}
\tilde{F}:\left(X_{G}, A_{G}\right) \times I & \rightarrow\left(Y_{G}, B_{G}\right) \\
([m, x], t) & \mapsto[m, F(x, t)],
\end{aligned}
$$

a qual satisfaz, para todo $[m, x] \in X_{G}$

$$
\begin{aligned}
& \tilde{F}([m, x], 0)=[m, F(x, 0)]=[m, f(x)]=f_{G}([m, x]) \\
& \tilde{F}([m, x], 1)=[m, F(x, 1)]=\left[m, f^{\prime}(x)\right]=f_{G}^{\prime}([m, x])
\end{aligned}
$$

Portanto, $\tilde{F}$ é uma homotopia entre $f_{G}$ e $f_{G}^{\prime}$.

PROPOSIÇÃo 2.3.28. Seja Top ${ }^{2}$ a categoria dos pares topológicos. Existe um funtor covariante, chamado Funtor construção de Borel,

$$
{ }_{G}: \mathbf{G}-\mathbf{T o p} \boldsymbol{p}^{2} \longrightarrow \boldsymbol{T o p} \boldsymbol{p}^{2},
$$

o qual associa a cada $G$-par $(X, A)$, um par de Borel $\left(X_{G}, A_{G}\right)$ e, para cada aplicação $G$ equivariante $f:(X, A) \rightarrow(Y, B)$, associa um aplicação de pares de Borel $f_{G}:\left(X_{G}, A_{G}\right) \rightarrow$ $\left(Y_{G}, B_{G}\right)$.

Demonstração: $\operatorname{Seja} \operatorname{Id}_{(X, A)}:(X, A) \rightarrow(X, A)$ a identidade em G-Top ${ }^{2}$,

$$
\begin{aligned}
{\left[\operatorname{Id}_{(X, A)}\right]_{G} } & =\overline{\operatorname{Id}_{E G} \times \operatorname{Id}_{(X, A)}}=\overline{\operatorname{Id}_{(E G \times X, E G \times A)}} \\
& =\operatorname{Id}_{\left(\frac{E G \times X}{G}, \frac{E G \times A}{G}\right)}=\operatorname{Id}_{\left(X_{G}, A_{G}\right)} .
\end{aligned}
$$

Segue que $\operatorname{Id}_{G}=\operatorname{Id}_{\left(X_{G}, A_{G}\right)}$. Além disso, dadas aplicações $G$-equivariantes

$$
\begin{aligned}
f:(X, A) & \rightarrow(Y, B) \text { e } f^{\prime}:(Y, B) \rightarrow(Z, C) \\
\left(f^{\prime} \circ f\right)_{G} & =\overline{\operatorname{Id}_{E G} \times\left(f^{\prime} \circ f\right)}=\overline{\left(\operatorname{Id}_{E G} \times f^{\prime}\right) \circ\left(\operatorname{Id}_{E G} \times f\right)} \\
& =\overline{\left(\operatorname{Id}_{E G} \times f^{\prime}\right)} \circ \overline{\operatorname{Id}_{E G} \times f}=f_{G}^{\prime} \circ f_{G} .
\end{aligned}
$$

Portanto, $-{ }_{G}$ é um funtor covariante.

Este funtor leva $G$-pares paracompactos $(X, A)$ em pares paracompactos $\left(X_{G}, A_{G}\right)$. De fato, $X_{G}$ é paracompacto, pela Observação 2.3 .22 e $A_{G}$ é fechado, desde que $E G \times A$ é fechado e a aplicação $\pi: E G \times A \rightarrow A_{G}$ é fechada, segundo a Proposição 2.2.21. 


\section{Índice cohomológico de um G-espaço: G-índice}

O objetivo principal deste capítulo é apresentar um estudo detalhado da teoria de $G$ índice desenvolvida por Fadell e Husseini em [20] e [21]. Possivelmente, Yang [54] foi o primeiro a definir um $\mathbb{Z} / 2$-índice (co)homológico de forma explícita, para espaços com ação livre de $\mathbb{Z} / 2$. Tal índice, que está conectado com o conceito de gênus, possui diversas aplicações em diferentes áreas da matemática.

Para ações livres de grupos finitos arbitrários, tanto os índices homológicos e geométricos foram primeiramente introduzidos por Shvarts [47],[48] e Conner-Floyd [15],[16]. Posteriormente, para o estudo dos espaços com ação de um grupo compacto de Lie, tornou-se necessária a definição de índices mais gerais, particularmente em aplicações a equações diferenciais não lineares, bifurcações e pontos críticos de aplicações $G$-equivariantes. Muitos autores têm dedicado seus estudos às definições e aplicações dos índices de tais $G$-espaços, vide por exemplo, [21] e as referências neste artigo.

Antes da publicação do trabalho de Fadell e Husseini [20], haviam sido considerados apenas índices numéricos, os quais associam a cada $G$-espaço, um número inteiro positivo (ou o símbolo $\infty$ ), com o objetivo de analisar a existência ou não de aplicações $G$-equivariantes. Tais resultados, muitas vezes conduzem a consequências importantes. Por exemplo, o teorema clássico de Borsuk-Ulam, o qual afirma que toda aplicação contínua de uma esfera $n$-dimensional no espaço euclidiano $\mathbb{R}^{n}$ colapsa pelo menos um par de pontos antípodas, é equivalente à afirmação de que não existe uma aplicação $\mathbb{Z} / 2$ equivariante $S^{n} \rightarrow S^{n-1}$, onde $\mathbb{Z} / 2$ age livremente sobre as esferas, via uma involução $\mathbb{Z} / 2$-equivariante.

No caso de grupos com anéis de cohomología suficientemente simples (por exemplo, álgebras polinomiais de uma variável), é conveniente utilizar índices numéricos. No entanto, invariantes numéricos são insuficientes no caso de grupos mais complexos, como por exemplo o toro e o p-toro. Neste contexto, Fadell e Husseini [20] introduziram o chamado Índice de valor ideal: associando a cada $G$-par paracompacto $(X, A)$, um ideal $\operatorname{Ind}^{G}(X, A ; \mathbb{K})$ do anel de cohomologia $\check{H}^{*}(B G ; \mathbb{K})$, onde $\check{H}^{*}$ é a cohomologia de Čech com coeficientes em um corpo $\mathbb{K}$ e $B G$ é o espaço classificante do grupo $G$. O ideal Ind ${ }^{G}(X ; \mathbb{K})$ é considerado como o kernel do homomorfismo entre as cohomologias $G$-equivariantes induzidas pela aplicação de $X$ em espaço contendo apenas um ponto. É fácil mostrar 
que, se existe uma aplicação $G$-equivariante de $X$ em $Y$, então $\operatorname{Ind}^{G}(Y ; \mathbb{K}) \subset \operatorname{Ind}^{G}(X ; \mathbb{K})$. As seções estão organizadas como segue. Na Seção 3.1, apresentamos a construção da cohomologia $G$-equivariante de Borel, usando a construção de Borel e a cohomologia de Čech. Na Subeção 3.1.1, induzimos sobre os módulos de cohomologia $G$-equivariante de Borel, uma estrutura de módulo graduado sobre o anel graduado $\check{H}^{*}(B G ; R)$. Tendo estruturado este módulo, podemos associar seu ideal anulador (graduado) e, usando este ideal, apresentamos na Seção 3.2, a definição do G-índice de Fadell e Husseini, tanto de valor ideal, como de valor numérico. Nas Subseções 3.2.1 e 3.2.2, estudamos o comportamento deste ideal. Para isto, consideramos primeiramente o caso relativo, com suas respectivas propriedades e, como um caso particular, estudamos o caso absoluto e suas propriedades. Para as aplicações no Capítulo 4, nosso maior interese é o caso particular do $G$-índice (absoluto). Na Subseção 3.3 apresentamos o $G$-índice do produto e do join. Finalmente, na Seção 3.4, relacionamos o $G$-índice numérico aos índices de Yang e Fadell-Rabinowitz. Além disso, apresentamos técnicas para o cálculo do $O(k)$-índice da variedade de Stiefel, onde $O(k)$ é o grupo ortogonal.

\subsection{Cohomologia $G$-equivariante de Borel}

Na aplicação da teoria de cohomologia para o estudo de grupos de transformações compactos, um formalismo natural e conveniente é definir uma teoria de cohomologia $G$ equivariante na categoria G-Top ${ }_{p c}^{2}$, que efetivamente reflita o comportamento cohomológico do $G$-espaço e da $G$-ação. Um dos objetivos desta seção é a construção desta cohomologia, que será a base para nossas próximas definições. Especificamente, descreveremos os principais resultados sobre a Cohomologia equivariante de Borel que utilizaremos durante o desenvolvimento deste trabalho. Para isso, usaremos os fatos da Teoria de cohomologia de Čech, descrita no Capítulo 1. Uma apresentação detalhada sobre esta teoria, pode ser encontrada em [10].

LEMA 3.1.1. Se F: $\mathscr{C} \rightarrow \mathscr{D}$ e $F^{\prime}: \mathscr{D} \rightarrow \mathscr{E}$ são funtores covariantes e contravariantes, respectivamente, então a composição $F^{\prime} \circ F: \mathscr{C} \rightarrow \mathscr{E}$ é um funtor contravariante.

Demonstração: Inicialmente temos que $\left(F^{\prime} \circ F\right)(\mathrm{Id})=F^{\prime}(\mathrm{Id})=\mathrm{Id}$. Agora, se $f_{1}, f_{2}$ são morfismos na categoria $\mathscr{C}$, então

$$
\begin{aligned}
\left(F^{\prime} \circ F\right)\left(f_{1} \circ f_{2}\right) & =F^{\prime}\left(F\left(f_{1}\right) \circ F\left(f_{2}\right)\right) \\
& =F^{\prime}\left(F\left(f_{2}\right)\right) \circ F^{\prime}\left(F\left(f_{1}\right)\right) \\
& =\left(F^{\prime} \circ F\right)\left(f_{2}\right) \circ\left(F^{\prime} \circ F\right)\left(f_{1}\right) .
\end{aligned}
$$

Portanto, $F^{\prime} \circ F$ é um funtor contravariante.

De agora em diante, $G$ denotará um grupo compacto de Lie fixado e $R$ um anel comutativo com identidade, a menos que se diga o contrário. Sejam $\check{H}^{q}: \mathbf{T o p}^{2} \rightarrow \mathbf{R}-\mathbf{m o d}$ o $q$-funtor cohomologia de Čech, com coeficientes no anel $R$ (Vide Teorema 1.3.18) e $-_{G}:$ G-Top ${ }^{2} \rightarrow$ Top $^{2}$ o funtor Construção de Borel. Desde que $-_{G}$ é covariante e $\check{H}^{q}$ é contravariante segue do Lema 3.1.1 que a composição é um funtor contravariante. Com isso temos a seguinte definição: 
DEFINIÇÃO 3.1.2. O funtor contravariante deduzido da composição

$$
\text { G-Top }{ }^{2} \stackrel{-{ }_{G}}{\longrightarrow} \text { Top }^{2} \stackrel{\check{H}^{q}}{\longrightarrow} \text { R-mod }
$$

denotado por $H_{G}^{q}$, é chamado o q-funtor cohomologia $G$-equivariante de Borel. Ou seja, a cada $G$-par $(X, A)$, associamos o $R$-módulo

$$
H_{G}^{q}(X, A ; R)=\check{H}^{q}\left(X_{G}, A_{G} ; R\right)
$$

e, a cada aplicação de pares $G$-equivariante $f:(X, A) \rightarrow(Y, B)$, o $R$-homomorfismo

$$
f_{G}^{*}=\left(\check{f}_{G}\right)^{*}: H_{G}^{q}(Y, B ; R) \rightarrow H_{G}^{q}(X, A ; R) .
$$

Da seção 1.3.3, temos que o funtor $\check{H}^{q}$ satisfaz os axiomas de Eilenberg-Steenrod e, desde que o funtor $-_{G}$ preserva homotopias (Vide Proposição 2.3.27), segue da definição do funtor $H_{G}^{q}$, que este também satisfaz tais axiomas, exceto o axioma da dimensão. Apresentaremos a seguir, a prova dos axiomas da invariância por homotopia e da exatidão, os quais serão necessários para as próximas demonstrações, as demais serão omitidas.

PROPOSIÇÃo 3.1.3. InVARIÂNCIA POR HOMOTOPIA: Se $f_{1}, f_{2}:(X, A) \rightarrow(Y, B)$ são aplicações G-homotópicas, então $f_{1 G}^{*}=f_{2 G}^{*}$.

Demonstração: Se $f_{1}, f_{2}$ são $G$-homotópicas então da Proposição 2.3.27 $f_{1 G}$ e $f_{2 G}$ são homotópicas. Segue do axioma da invariância por homotopia na cohomologia de Čech que $f_{1 G}^{*}=\left(\check{f}_{1 G}\right)^{*}=\left(\check{f}_{2 G}\right)^{*}=f_{2 G}^{*}$.

PROPOSIÇÃo 3.1.4. EXATIDÃO: Dado o $G$-par $(X, A)$, existe um $R$-homomorfismo $\delta: H_{G}^{q}(A ; R) \rightarrow H_{G}^{q+1}(X, A ; R)$ que comuta com os homomorfismos induzidos em cohomologia $G$-equivariante de Borel e a sequência

$$
\cdots \stackrel{\delta}{\longrightarrow} H_{G}^{q+1}(X, A ; R) \stackrel{J_{G}^{*}}{\longrightarrow} H_{G}^{q+1}(X ; R) \stackrel{l_{G}^{*}}{\longrightarrow} H_{G}^{q+1}(A ; R) \stackrel{\delta}{\longrightarrow} \cdots
$$

é exata, onde $\imath:(A, B) \rightarrow(X, B), \jmath:(X, B) \rightarrow(X, A)$ são as inclusões.

Demonstração: Seja $\left(X_{G}, A_{G}\right)$ o par de Borel do $G$-par $(X, A)$. Pelo axioma da exatidão da cohomologia de Čech, existe um $R$-homomorfismo

$$
\delta: \check{H}^{q}\left(A_{G} ; R\right) \rightarrow \check{H}^{q+1}\left(X_{G}, A_{G} ; R\right)
$$

o qual comuta com os homomorfismos induzidos na cohomologia de Čech e tal que a seguinte sequência longa é exata

$$
\cdots \stackrel{\delta}{\longrightarrow} \check{H}^{q+1}\left(X_{G}, A_{G} ; R\right) \stackrel{\left(\check{J_{G}}\right)^{*}}{\longrightarrow} \check{H}^{q+1}\left(X_{G}, B_{G} ; R\right) \stackrel{\left(\check{\iota_{G}}\right)^{*}}{\longrightarrow} \check{H}^{q+1}\left(A_{G}, B_{G} ; R\right) \stackrel{\delta}{\longrightarrow},
$$

onde $\imath_{G}:\left(A_{G}, B_{G}\right) \rightarrow\left(X_{G}, B_{G}\right)$ e $\jmath:\left(X_{G}, B_{G}\right) \rightarrow\left(X_{G}, A_{G}\right)$ são as inclusões. Portanto, usando a definição do funtor $H_{G}^{q}$, segue o resultado.

Anteriormente, frisamos que a cohomologia $G$-equivariante de Borel não satisfaz o axioma da dimensão, pois o $R$-módulo desta cohomologia, no caso de um $G$-espaço que contém apenas um ponto, não é necessariamente o anel $R$ no nível zero e o módulo nulo nos níveis $q>0$, como mostra a seguinte proposição: 
PROPOSIÇão 3.1.5. . Se $\left\{x_{o}\right\}$ for um G-espaço com $G$-ação trivial, então

$$
H_{G}^{q}\left(\left\{x_{0}\right\}, R\right) \cong \check{H}^{q}(B G ; R) .
$$

Demonstração: Consideremos $\pi: E G \rightarrow B G$ a aplicação projeção do $G$-fibrado universal. Como $E G \times\left\{x_{0}\right\}$ é homeomorfo a $E G$, seja $\pi^{\prime}: E G \times\left\{x_{0}\right\} \approx E G \stackrel{\pi}{\rightarrow} B G$ a aplicação quociente. Consideremos também a decomposição

$$
\left\{\pi^{\prime-1}(G m): m \in E G\right\}=\left\{\left[m, x_{0}\right]: m \in E G\right\}=E G \times_{G}\left\{x_{0}\right\},
$$

munido da topologia quociente co-induzida pela aplicação natural

$$
p: E G \times\left\{x_{0}\right\} \rightarrow E G \times_{G}\left\{x_{0}\right\} .
$$

Logo, usando resultados de topologia quociente, temos que a aplicação quociente $\pi^{\prime}$ : $E G \times\left\{x_{0}\right\} \rightarrow B G$ determina um homeomorfismo $\psi: E G \times_{G}\left\{x_{0}\right\} \rightarrow B G$ tal que o seguinte diagrama comuta

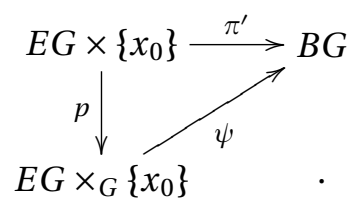

Portanto, $E G \times_{G}\left\{x_{0}\right\} \approx B G$ e, segue que

$$
H_{G}^{q}\left(\left\{x_{0}\right\}, R\right)=\check{H}^{q}\left(E G \times_{G}\left\{x_{0}\right\}, R\right) \cong \check{H}^{q}(B G ; R) .
$$

Se $X$ for um espaço paracompacto e se a $G$-ação sobre $X$ for livre, temos que o espaço de Borel $X_{G}$ e o espaço de órbitas $X / G$ possuem seus $R$-módulos de cohomologia de Čech isomorfos, como mostra a seguinte:

PROPOSIÇÃo 3.1.6. Seja X um G-espaço paracompacto com G-ação livre. Então,

$$
H_{G}^{q}(X ; R) \cong \check{H}^{q}(X / G ; R) .
$$

Demonstração: Provaremos inicialmente que aplicação $\overline{\pi_{2}}: X_{G} \rightarrow X / G$ induzida da projeção na segunda coordenada $\pi_{2}: E G \times X \rightarrow X$ entre os espaços órbitas, é uma projeção de algum $G$-fibrado. Com feito, a aplicação $\overline{\pi_{2}}$ é sobrejetiva, pois dada a órbita $G x$ do ponto $x \in X$,

$$
\begin{gathered}
G x=G\left(\pi_{2}(m, x)\right)=\overline{\pi_{2}}([m, x]) \mathrm{e} \\
\bar{\pi}^{-1}(G x)=\{[m, y]: G y=G x\}=\{[m, g x]: m \in E G, g \in G\}=(G x)_{G}
\end{gathered}
$$

Pelo teorema 2.3.5, a quíntupla $(X, \pi, X / G, G, G)$ é um $G$-fibrado principal, então para todo $x \in X, G x \approx G$ o que implica que $\bar{\pi}_{2}^{-1}(G x) \approx G_{G}$. Por outro lado, se considerarmos a aplicação $\mu: E G \times G \rightarrow E G$ definida por $\mu(m, g)=g^{-1} m$, temos que $\mu^{-1}(m)=\{(g m, g)$ : $g \in G\}=[y, g] \in G_{G}$. Consideremos também a decomposição

$$
\left\{\mu^{-1}(m): m \in E G\right\}=\{[y, g]: y \in E G, g \in G\}=G_{G}
$$


munida a topologia quociente co-induzida pela aplicação natural $p: E G \times G \rightarrow G_{G}$. Logo, usando resultados de topologia quociente, temos que a aplicação quociente $\mu: E G \times G \rightarrow$ $E G$ determina um homeomorfismo $\psi: G_{G} \rightarrow B G$ tal que o seguinte diagrama comuta

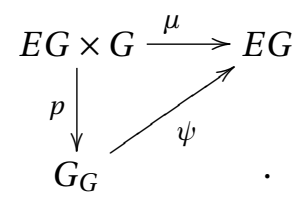

Então, para todo $x \in X$ a fibra $\bar{\pi}^{-1}(G x) \approx G_{G} \approx E G$ e, portanto, $\left(X_{G}, \overline{\pi_{2}}, X / G, E G, G\right)$ é um $G$-fibrado com fibra $E G$ contrátil (Teorema 2.3.16). Pelo Teorema de Leray-Hirsch ([10, Proposição 1.14, pg. 181]), desde que $\check{H}^{q}(E G ; R)=0$, para todo $q>0$, concluimos que

$$
\check{H}^{q}\left(X_{G} ; R\right)=H_{G}^{q}(X ; R) \cong \check{H}^{q}(X / G ; R) \otimes \check{H}^{0}(E G ; R) \cong \check{H}^{q}(X / G ; R) .
$$

A continuidade da cohomologia de Čech, induz uma continuidade sobre a cohomologia $G$-equivariante de Borel, como mostra o seguinte

teorema 3.1.7. (Teorema da Continuidade) Seja $(X, A)$ um $G$-par paracompacto sendo $X$ um subconjunto fechado $G$-invariante do $G$-espaço paracompacto $Y$. Então, $\left\{H_{G}^{q}(U, V ; R), l_{G}^{*}, \mathscr{P}\right\}$ é um sistema direto, onde $\mathscr{P}$ é a coleção de todos os pares de vizinhanças fechadas $G$-invariantes $(U, V)$ de $(X, A)$ e $\imath_{G}^{*}$ é o homomorfismo induzido da inclusão $\iota:\left(U^{\prime}, V^{\prime}\right) \rightarrow(U, V)$ na cohomologia $G$-equivariante de Borel. Além disso, existe um isomorfismo

$$
\varpi_{q}: \lim _{(U, V) \in \mathscr{N}} H_{G}^{q}(U, V ; R) \rightarrow H_{G}^{q}(X, A ; R),
$$

ou seja,

$$
H_{G}^{q}(X, A ; R) \cong \lim _{\substack{\overrightarrow{(}, V) \in \mathcal{N}}} H_{G}^{q}(U, V ; R) .
$$

Mais geralmente, existe um isomorfismo

$$
\lim _{(U, V) \in \mathscr{N}} H_{G}^{*}(U, V ; R) \cong H_{G}^{*}(X, A ; R) .
$$

Sejam $\left(X, A_{1}\right),\left(X, A_{2}\right) G$-pares tais que $\left\{A_{1}, A_{2}\right\}$ é um par excisivo de $A_{1} \cup A_{2}$. Assim, $\left\{A_{1 G}, A_{2 G}\right\}$ também é um par excisivo de $A_{1 G} \cup A_{2 G}$. Portanto, segue da Definição 1.3.22, a existência do produto cup em cohomologia de Čech, ou seja, a aplicação

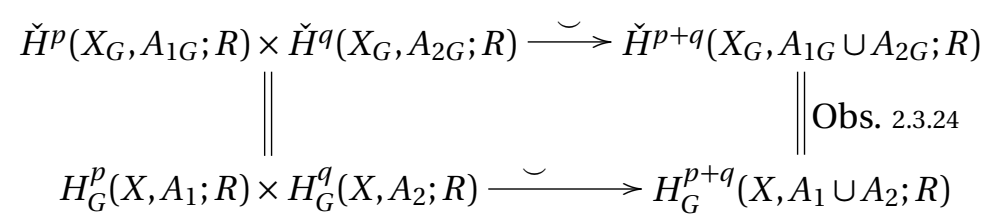

Portanto, existe o produto cup na Cohomologia $G$-equivariante de Borel, satisfazendo as seguintes propriedades, as quais se deduzem das propriedades da cohomologia de Čech. 
PROPOSIÇÃO 3.1.8.

1. (Linearidade) Sejam $f:\left(X, A_{1} \cup A_{2}\right) \rightarrow\left(Y, B_{1} \cup B_{2}\right), f_{1}:\left(X, A_{1}\right) \rightarrow\left(Y, B_{1}\right), e$ $f_{2}:\left(X, A_{2}\right) \rightarrow\left(Y, B_{2}\right)$ aplicações pares $G$-equivariantes tais que $f_{1}(x)=f_{2}(x)=f(x)$ para todo $x \in X$. Se $u \in H_{G}^{p}\left(Y, B_{1} ; R\right)$ e $v \in H_{G}^{q}\left(Y, B_{2} ; R\right)$, então em $H_{G}^{p+q}\left(X, A_{1} \cup A_{2} ; R\right)$ temos

$$
f_{G}^{*}(u \smile v)=f_{1 G}^{*}(u) \smile f_{2 G}^{*}(v) .
$$

Em particular, para toda aplicação $G$-equivariante $f: X \rightarrow Y$

$$
f_{G}^{*}(u \smile v)=f_{G}^{*}(u) \smile f_{G}^{*}(v) .
$$

2. (Identidade) Para algum $u \in H_{G}^{q}(X, A ; R)$, temos que $1 \smile u=u \smile 1=u$, com $1 \in H_{G}^{0}(X ; R)$.

3. (Associatividade) Sejam $u_{1} \in H_{G}^{p}\left(X, A_{1} ; R\right), u_{2} \in H_{G}^{q}\left(X, A_{2} ; R\right)$ e $u_{3} \in H_{G}^{r}\left(X, A_{3} ; R\right)$. Então, em $H_{G}^{p+q+r}\left(X, A_{1} \cup A_{2} \cup A_{3} ; R\right)$ temos

$$
u_{1} \smile\left(u_{2} \smile u_{3}\right)=\left(u_{1} \smile u_{2}\right) \smile u_{3} .
$$

4. (Anticomutatividade) Sejam $u \in H_{G}^{p}\left(X, A_{1} ; R\right)$ e $v \in H_{G}^{q}\left(X, A_{2} ; R\right)$, então em $H_{G}^{p+q}\left(X, A_{1} \cup A_{2} ; R\right)$ temos

$$
u \smile v=(-1)^{p q} v \smile u .
$$

Corolario 3.1.9. Para todo $u \in H_{G}^{p}\left(X, A_{1}\right) e 0 \in H_{G}^{q}\left(X, A_{2} ; R\right)$ o elemento neutro, então

$$
u \smile 0=0=0 \smile u
$$

Demonstração: Seja $v \in H_{G}^{q}\left(X, A_{2} ; R\right)$. Usando a bilinearidade do produto cup, tem-se

$$
u \smile 0=u \smile(v-v)=u \smile v-u \smile v=0
$$

Finalizando os resultados sobre a cohomologia $G$-equivariante de Borel, apresentamos a seguir a sequência de Mayer-Vietoris nesta cohomologia equivariante.

PRoposição 3.1.10. Sejam $\left\{\left(X_{1}, A_{1}\right),\left(X_{2}, A_{2}\right)\right\}$ um par excisivo de $(X, A)=\left(X_{1} \cup X_{2}, A_{1} \cup A_{2}\right)$. Dados $G$-pares $\left(X_{1}, A_{1}\right),\left(X_{2}, A_{2}\right) e(X, A)$, então a seguinte sequência

$$
\cdots \rightarrow H_{G}^{q}(X, A ; R) \stackrel{\zeta}{\rightarrow} H_{G}^{q}\left(X_{1}, A_{1} ; R\right) \oplus H_{G}^{q}\left(X_{2}, A_{2} ; R\right) \stackrel{\eta}{\rightarrow} H_{G}^{q}\left(X_{1} \cap X_{2}, A_{1} \cap A_{2} ; R\right) \rightarrow \cdots
$$

é exata, onde $\zeta=\left(i_{0 G}^{*},-i_{1 G}^{*}\right), \eta=\ell_{0 G}^{*}+\ell_{1 G}^{*} \operatorname{com} i_{n}:\left(X_{n}, A_{n}\right) \rightarrow(X, A), n=0,1$, $\ell_{0}:\left(X_{1} \cap X_{2}, A_{1} \cap A_{2}\right) \rightarrow\left(X_{1}, A_{1}\right) e \ell_{1}:\left(X_{1} \cap X_{2}, A_{1} \cap A_{2}\right) \rightarrow\left(X_{2}, A_{2}\right)$. 


\subsubsection{Estrutura de módulo graduado sobre $\check{H}^{*}(B G ; R)$}

Definidos os módulos de cohomologia equivariante de Borel, nosso próximo passo é construir sobre esses módulos uma estrutura algébrica, cujo comportamento cohomológico seja descrito pelas informações obtidas a partir da cohomologia de Čech dos espaços classificantes. Para isso, começamos com a seguinte definição de anel graduado (Vide [1, pg. 106]).

DEFINIÇão 3.1.11. Seja $R$ um anel. Dizemos que $R$ é um anel graduado, se existe uma família $\left\{R_{n}\right\}_{n \geq 0}$ de subgrupos aditivos de $R$ tal que $R=\oplus R_{n}$ e $R_{m} R_{n} \subset R_{m+n}$ para cada $m, n \geq 0$.

EXEMPLO 3.1.12. Seja $X$ um espaço topológico. Consideremos a soma direta dos $R$-módulos $\check{H}^{p}(X ; R)$

$$
\begin{aligned}
\check{H}^{*}(X ; R)= & \bigoplus_{p \geq 0} \check{H}^{p}(X ; R) \\
= & \left\{\Sigma \lambda_{p}: \lambda_{p} \in \check{H}^{p}(X ; R) \text { e } \lambda_{p} \neq 0\right. \text { apenas para } \\
& \text { um número finito de } p \geq 0\}
\end{aligned}
$$

com a estrutura de soma e multiplicação de $R$-módulos definida por: dados $\lambda=\Sigma \lambda_{l}, v=\Sigma v_{p} \in \check{H}^{*}(X ; R)$ e $r \in R$

$$
\lambda+v=\Sigma\left(\lambda_{p}+v_{p}\right), \quad r \lambda=\Sigma r \lambda_{l} .
$$

Dados $\lambda=\Sigma \lambda_{l}$ e $v=\Sigma v_{p}$ elementos de $\check{H}^{*}(X ; R)$, o produto de anel sobre $\check{H}^{*}(X ; R)$ é dada por:

$$
\lambda \smile v=\Sigma_{l}\left(\Sigma_{p}\left(\lambda_{l} \smile v_{p}\right)\right)=\Sigma_{l, p}\left(\lambda_{l} \smile v_{p}\right) \in \check{H}^{*}(B G ; R)
$$

onde $\smile: \check{H}^{l} \times \check{H}^{p} \rightarrow \check{H}^{l+p}$ é o produto cup. Desde que $\lambda_{l} \smile v_{p} \in \check{H}^{l+p}(X ; R)$ para todo $\lambda_{l} \in \check{H}^{l}(X ; R)$ e $v_{p} \in \check{H}^{p}(X ; R)$, segue que o $R$-módulo $\check{H}^{*}(X ; R)$ tem uma estrutura de anel graduado. Usando a propriedade da anticomutatividade (Proposição 3.1.8) do produto cup, vemos que:

$$
\lambda \smile v=\Sigma_{l, p}\left(\lambda_{l} \smile v_{p}\right)=\Sigma_{l p}(-1)^{l p}\left(\lambda_{l} \smile v_{p}\right) .
$$

Se $\lambda=\Sigma \lambda_{l}$, com $\lambda_{0}=1 \in \check{H}^{0}(X ; R) \cong R$ e $\lambda_{l}=0$ para todo $l>0$, então segue da propriedade da identidade (Proposição 3.1.8) e do corolário 3.1.9 do produto cup que

$$
1 \smile v=\Sigma_{l, p}\left(\lambda_{l} \smile v_{p}\right)=\Sigma_{p}\left(\Sigma_{l}\left(\lambda_{l} \smile v_{p}\right)=\Sigma_{p}\left(1 \smile v_{p}\right)=\Sigma_{p} v_{p}=v .\right.
$$

Denotaremos simplesmente o produto $\lambda \smile v$ pela soma $\Sigma\left(\lambda_{l} \smile v_{p}\right)$ omitindo os índices $l, p$ e, denotaremos por 1 o elemento $\Sigma \lambda_{l} \operatorname{com} \lambda_{0}=1$ e $\lambda_{l}=0$ para todo $l>0$, chamado elemento identidade do produto do anel graduado $\check{H}^{*}(X ; R)$.

OBSERVAÇÃo 3.1.13. Como caso particular do exemplo anterior temos que $\check{H}^{*}(B G ; R)$ é um anel graduado.

DEFINIÇÃo 3.1.14. Seja $R$ um anel graduado e $M$ um $R$-módulo. Dizemos que $M$ é um R-módulo graduado se existe uma familia $\left\{M_{n}\right\}$ de subgrupos aditivos de $M$ tal que $M=\oplus M_{n}$ e $R_{m} M_{n} \subset M_{m+n}$ para todo $m, n \geq 0$. 
DEFINIÇão 3.1.15. Se $M$ e $N$ são $R$-módulos graduados, um homomorfismo de R-módulos graduados é um homomorfismo de $R$-módulos $f: M \rightarrow N$ tal que $f\left(M_{n}\right) \subset N_{n}$ para todo $n \geq 0$.

Seja $(X, A)$ um $G$-par paracompacto. Como no exemplo 3.1.12, consideremos o $R$-módulo soma direta dos $R$-módulos $H_{G}^{q}(X, A ; R)$;

$$
\begin{aligned}
H_{G}^{*}(X, A ; R)= & \bigoplus_{q \geq 0} H_{G}^{q}(X, A ; R) \\
= & \left\{\Sigma x_{q}: x_{q} \in H_{G}^{q}(X, A ; R) \text { e } x_{q} \neq 0\right. \text { apenas para } \\
& \text { um número finito de } q \geq 0\}
\end{aligned}
$$

com a estrutura de soma e multiplicação análoga ao do exemplo 3.1.12.

$\mathrm{O}$ objetivo agora, é mostrar que o $R$-módulo $H_{G}^{*}(X, A ; R)$ admite uma estrutura de $\breve{H}^{*}(B G ; R)$-módulo graduado. Para isso, devemos definir uma multiplicação especial do anel graduado $\breve{H}^{*}(B G ; R)$ sobre o $R$-módulo $H_{G}^{*}(X, A ; R)$, a qual descreveremos a seguir. Seja $\lambda=\Sigma \lambda_{p} \in \check{H}^{*}(B G ; R)$ e consideremos o homomorfismo

$$
\oplus \check{q}_{X}^{*}: \check{H}^{*}(B G ; R) \rightarrow H_{G}^{*}(X ; R), \quad\left(\oplus \check{q}_{X}^{*}\right)(\lambda)=\Sigma \check{q}_{X}^{*}\left(\lambda_{p}\right),
$$

onde $\check{q}_{X}^{*}: \check{H}^{p}(B G ; R) \rightarrow H_{G}^{p}(X ; R)$ é o homomorfismo induzido na cohomologia de Čech da aplicação classificante $q_{X}: X_{G} \rightarrow B G$ (Observação 2.3.22). Denotaremos o homomorfismo $\oplus \check{q}_{X}^{*}$ simplesmente por $q_{X}^{*}$. Assim, dados $\lambda=\Sigma \lambda_{p} \in \check{H}^{*}(B G ; R)$ e $x=\Sigma x_{q} \in$ $H_{G}^{*}(X, A ; R)$ definimos

$$
\lambda x=q_{X}^{*}(\lambda) \smile x=\Sigma_{p, q}\left(\check{q}_{X}^{*}\left(\lambda_{p}\right) \smile x_{q}\right),
$$

onde $\smile: H_{G}^{p}(X, R) \times H_{G}^{q}(X, A ; R) \rightarrow H_{G}^{p+q}(X, A ; R)$ é o produto cup.

TEOREMA 3.1.16. Se $(X, A)$ é um $G$-par paracompacto, então o $R$-módulo $H_{G}^{*}(X, A ; R)$ possui estrutura de módulo graduado sobre o anel graduado $\check{H}^{*}(B G ; R)$, determinada pela multiplicação:

$$
\lambda x=q_{X}^{*}(\lambda) \smile x=\Sigma_{p, q}\left(\check{q}_{X}^{*}\left(\lambda_{p}\right) \smile x_{q}\right) .
$$

Demonstração: Com efeito:

1. Sejam $\lambda=\Sigma \lambda_{p} \in \check{H}^{*}(B G ; R), x=\Sigma x_{q}, y=\Sigma y_{q} \in H_{G}^{*}(X, A ; R)$. Da operação soma na soma direta temos que $x+y=\Sigma\left(x_{q}+y_{q}\right)$. Desde que o produto cup é uma aplicação bilinear, segue que

$$
\begin{aligned}
\lambda(x+y) & =\check{q}_{X}^{*}(\lambda) \smile(x+y) \\
& =\Sigma_{p, q}\left[\check{q}_{X}^{*}\left(\lambda_{p}\right) \smile\left(x_{q}+y_{q}\right)\right] \\
& =\Sigma_{p, q}\left[\left(\check{q}_{X}^{*}\left(\lambda_{p}\right) \smile x_{q}\right)+\left(\check{q}_{X}^{*}\left(\lambda_{p}\right) \smile y_{q}\right)\right] \\
& =\Sigma_{p, q}\left(\check{q}_{X}^{*}\left(\lambda_{p}\right) \smile x_{q}\right)+\Sigma_{p, q}\left(\check{q}_{X}^{*}\left(\lambda_{p}\right) \smile y_{q}\right) \\
& =\left(\check{q}_{X}^{*}(\lambda) \smile x\right)+\left(\check{q}_{X}^{*}(\lambda) \smile y\right) \\
& =\lambda x+\lambda y .
\end{aligned}
$$


2. Sejam $\lambda=\Sigma \lambda_{p}, v=\Sigma v_{p} \in \check{H}^{*}(B G ; R), x=\Sigma x_{q} \in H_{G}^{*}(X, A ; R)$. Desde que $q_{X}^{*}$ é um $R$-homomorfismo e o produto cup é bilinear, temos que

$$
\begin{aligned}
(\lambda+v) x & =q_{X}^{*}(\lambda+v) \smile x \\
& =\Sigma_{p, q}\left(\check{q}_{X}^{*}\left(\lambda_{p}+v_{p}\right) \smile x_{q}\right) \\
& =\Sigma_{p, q}\left[\left(\check{q}_{X}^{*}\left(\lambda_{p}\right) \smile x_{q}\right)+\left(\check{q}_{X}^{*}\left(v_{p}\right) \smile x_{q}\right)\right] \\
& =\Sigma_{p, q}\left(\check{q}_{X}^{*}\left(\lambda_{p}\right) \smile x_{q}\right)+\Sigma_{p, q}\left(\check{q}_{X}^{*}\left(v_{p}\right) \smile x_{q}\right) \\
& =\left(q_{X}^{*}(\lambda) \smile x\right)+\left(q_{X}^{*}(v) \smile x\right) \\
& =\lambda x+v x .
\end{aligned}
$$

3. Sejam $\lambda=\Sigma \lambda_{l}, v=\Sigma v_{p} \in \check{H}^{*}(B G ; R), x=\Sigma x_{q} \in H_{G}^{*}(X, A ; \mathbb{K})$. Desde que o produto cup é linear e associativo (Proposição 3.1.8), segue que

$$
\begin{aligned}
(\lambda \smile v) x & =q_{X}^{*}(\lambda \smile v) \smile x \\
& =\left(q_{X}^{*}(\lambda) \smile q_{X}^{*}(v)\right) \smile x \\
& =q_{X}^{*}(\lambda) \smile\left(q_{X}^{*}(v) \smile x\right) \\
& =q_{X}^{*}(\lambda) \smile(v x) \\
& =\lambda(v x) .
\end{aligned}
$$

4. Sejam $x=\Sigma x_{q} \in H_{G}^{*}(X, A ; R)$ e $1=\Sigma \lambda_{p} \in \check{H}^{*}(B G ; R)$. Desde que $q_{X}^{*}(1)=1 \in$ $H_{G}^{*}(X, A ; \mathbb{K})$, segue da propriedade identidade do produto cup (Proposição 3.1.8) que,

$$
1 x=q_{X}^{*}(1) \smile x=\Sigma_{q}\left(\check{q}_{X}^{*}(1) \smile x_{q}\right)=\Sigma_{q}\left(1 \smile x_{q}\right)=\Sigma_{q} x_{q}=x .
$$

Além disso,

$$
\lambda_{p} x_{q}=\check{q}_{X}^{*}\left(\lambda_{p}\right) \smile x_{q} \in H_{G}^{p+q}(X, A ; R),
$$

para todo $x_{q} \in H_{G}^{q}(X, A ; R)$ e para todo $\lambda_{p} \in \check{H}^{p}(B G ; R)$. Portanto, $H_{G}^{*}(X, A ; R)$ é um $\check{H}^{*}(B G ; R)$-módulo graduado.

observaÇÃo 3.1.17. Se $f: X \rightarrow Y$ é uma aplicação equivariante, entre $G$-espaços paracompactos, então do Teorema 2.3.13, o seguinte diagrama

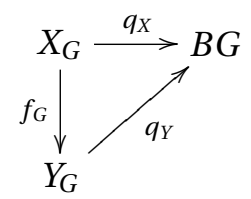

é homotopicamente comutativo, ou seja $q_{X}$ é homotópico a $q_{Y} f_{G}$ e, deste modo, $\check{q}_{X}^{*}=$ $\left(\check{q}_{Y} \circ\left(\check{f}_{G}\right)\right)^{*}: \check{H}^{p}(B G ; R) \rightarrow H_{G}^{p}(X ; R)$. Além disso existe um $R$-homomorfismo nas somas diretas $f_{G}^{*}=\oplus f_{G}^{*}: H_{G}^{*}(Y ; R) \rightarrow H_{G}^{*}(X ; R)$, dado por $f_{G}^{*}(y)=\Sigma f_{G}^{*}\left(y_{q}\right)$ onde $y=\Sigma y_{q}$. Logo

$$
\begin{aligned}
q_{X}^{*}\left(\Sigma \lambda_{p}\right) & =\Sigma \check{q}_{X}^{*}\left(\lambda_{p}\right) \\
& =\Sigma\left(\check{q}_{Y} \circ\left(\check{f}_{G}\right)\right)^{*}\left(\lambda_{p}\right) \\
& =\left(\check{q}_{Y} \circ\left(\check{f_{G}}\right)\right)^{*}\left(\Sigma \lambda_{p}\right)
\end{aligned}
$$

Portanto obtemos que $q_{X}^{*}=f_{G}^{*} \circ q_{Y}^{*}$. 
observaÇão 3.1.18. Para um para excesio $\left\{A_{1}, A_{2}\right\}$ de $A_{1} \cup A_{2}$, podemos definir o produto cup nas somas diretas da cohomologia $G$-equivariante de Borel da forma seguinte

$$
\smile: H_{G}^{*}\left(X, A_{1} ; R\right) \times H_{G}^{*}\left(X, A_{2} ; R\right) \rightarrow H_{G}^{*}\left(X, A_{1} \cup A_{2} ; R\right)
$$

dado por $u \smile v=\Sigma_{p, q}\left(u_{p} \smile v_{q}\right)$, onde $u=\Sigma u_{p}$ e $v=\Sigma v_{q}$. Esta aplicação bilinear satisfaz as propriedades da Proposição 3.1.8.

O próximo teorema, mostra que o $R$-homomorfismo induzido na cohomologia equivariante de Borel, é um $\check{H}^{*}(B G ; R)$-homomorfismo de módulos graduados.

TEOREMA 3.1.19. Seja $f:(X, A) \rightarrow(Y, B)$ uma aplicação equivariante entre G-pares paracompactos. Então, o R-homomorfismo

$$
f_{G}^{*}=\oplus f_{G}^{*}: H_{G}^{*}(Y, B ; R) \rightarrow H_{G}^{*}(X, A ; R),
$$

dado por $f_{G}^{*}(y)=\Sigma f_{G}^{*}\left(y_{q}\right)$ onde $y=\Sigma y_{q}$, é um homomorfismo de $\check{H}^{*}(B G ; R)$-módulos graduados.

Demonstração: Sejam $\lambda \in \check{H}^{*}(B G ; \mathbb{K})$ e $y \in H_{G}^{*}(Y, B ; \mathbb{K})$. Desde que o produto cup é linear (Proposição 3.1.8 e Observação 3.1.18), temos que

$$
\begin{aligned}
f_{G}^{*}(\lambda y) & =f_{G}^{*}\left(q_{Y}^{*}(\lambda) \smile y\right) \\
& =f_{G}^{*}\left(q_{Y}^{*}(\lambda)\right) \smile f_{G}^{*}(y) \\
& =\left(q_{Y} f_{G}\right)^{*}(\lambda) \smile f_{G}^{*}(y) .
\end{aligned}
$$

Segue da observação 3.1.17 que $q_{X}^{*}=\left(q_{Y} f_{G}\right)^{*}$. Logo, substituindo na equação (3.7), temos

$$
f_{G}^{*}(\lambda y)=q_{X}^{*}(\lambda) \smile f_{G}^{*}\left(y_{q}\right)=\lambda f_{G}^{*}(y) .
$$

Portanto, $f_{G}^{*}$ é um homomorfismo de $\check{H}^{*}(B G ; R)$-módulos graduados.

\subsection{G-índice cohomológico de Fadell e Husseini}

Nesta seção, faremos um estudo dos resultados desenvolvidos nos artigos de Fadell e Husseini [20] e [21]. Eles construiram um ideal em um determinado anel, chamado índice de valor ideal. A dimensão do quociente do anel com este ideal, foi denominada índice de valor numérico. Iniciaremos, definindo tal índice no caso relativo, ou seja para $G$-pares paracompactos e, posteriormente, definiremos este índice para o caso absoluto. A partir de agora, o anel de coeficientes dos módulos de cohomologia equivariante de Borel será um corpo $\mathbb{K}$.

\subsubsection{G-índice relativo}

Assim como definimos anéis graduados e módulos graduados sobre anéis graduados, podemos definir, de maneira análoga, um ideal graduado e, portanto, o anel quociente graduado. Tais definições são dadas a seguir. 
DEFINIÇão 3.2.1. Sejam $R$ um anel graduado e $I$ um ideal de $R$. Dizemos que $I$ é um ideal graduado, se $I=\oplus I_{n}$, onde $I_{n}=I \cap R_{n}$.

Se $R$ e $I$ forem graduados, então o anel quociente $R / I$ também é graduado. De fato,

$$
\frac{R}{I}=\bigoplus_{n \geq 0}\left(\frac{R}{I}\right)_{n}, \quad\left(\frac{R}{I}\right)_{n}=\left\{x+I, x \in R_{n}\right\}
$$

DEFINIÇÃo 3.2.2. Seja $(X, A)$ um $G$-par paracompacto. Definimos o G-índice cohomológico de Fadell e Husseini com valor ideal do $\operatorname{par}(X, A)$, o qual será denotado por

$$
\operatorname{Ind}^{G}(X, A ; \mathbb{K})
$$

como sendo o ideal anulador do $\check{H}^{*}(B G ; \mathbb{K})$-módulo $H_{G}^{*}(X, A ; \mathbb{K})$, ou seja :

$$
\operatorname{Ind}^{G}(X, A ; \mathbb{K})=\left\{\lambda \in \check{H}^{*}(B G ; \mathbb{K}): \lambda x=0 \text {, para todo } x \in H_{G}^{*}(X, A ; \mathbb{K})\right\} \text {. }
$$

Dados $\lambda, \lambda^{\prime} \in \operatorname{Ind}^{G}(X, A ; \mathbb{K})$, e $k \in \mathbb{K}$, desde que $H_{G}^{*}(X, A ; \mathbb{K})$ é um $\check{H}^{*}(B G ; \mathbb{K})$ - módulo graduado e $q_{X}^{*}$ é um $\mathbb{K}$-homomorfismo, temos que:

$$
\begin{aligned}
{\left[(k \lambda)+\lambda^{\prime}\right] x } & =(k \lambda) x+\lambda^{\prime} x \\
& =(k \lambda) x \\
& =q_{X}^{*}(k \lambda) \smile x \\
& =k\left(q_{X}^{*}(\lambda) \smile x\right) \\
& =k(\lambda x) \\
& =k(0) \\
& =0 .
\end{aligned}
$$

Portanto, $\operatorname{Ind}^{G}(X, A ; \mathbb{K})$ é um submódulo do $\mathbb{K}$-módulo (isto é, $\mathbb{K}$-espaço vetorial) $\check{H}^{*}(B G ; \mathbb{K})$ e, temos assim a seguinte

DEFINIÇ̃̃o 3.2.3. Seja $(X, A)$ um $G$-par paracompacto. Definimos o G-índice cohomológico de Fadell e Husseini com valor numérico do $\operatorname{par}(X, A)$, o qual será denotado por

$$
\left|\operatorname{Ind}^{G}(X, A ; \mathbb{K})\right| \text {, }
$$

como sendo a dimensão do $\mathbb{K}$-espaço vetorial obtido do quociente do espaço vetorial $\check{H}^{*}(B G ; \mathbb{K})$ pelo subespaço vetorial $\operatorname{Ind}^{G}(X, A ; \mathbb{K})$, isto é,

$$
\left|\operatorname{Ind}^{G}(X, A ; \mathbb{K})\right|=\operatorname{dim}_{\mathbb{K}}\left[\frac{\check{H}^{*}(B G, \mathbb{K})}{\operatorname{Ind}^{G}(X, A ; \mathbb{K})}\right] .
$$

Como o anel $\check{H}^{*}(B G ; \mathbb{K})$ é graduado, é interessante saber se o ideal $\operatorname{Ind}^{G}(X, A ; \mathbb{K})$ também possui estrutura graduada. Neste sentido, temos a seguinte 
PROPOSIÇÃo 3.2.4. O ideal $\operatorname{Ind}^{G}(X, A ; \mathbb{K})$ de $\check{H}^{*}(B G ; \mathbb{K})$ é graduado, com graduação dada por

$$
\operatorname{Ind}^{G}(X, A ; \mathbb{K})=\bigoplus_{p \geq 0} \operatorname{Ind}_{p}^{G}(X, A ; \mathbb{K}),
$$

onde as componentes são da forma

$$
\operatorname{Ind}_{p}^{G}(X, A ; \mathbb{K})=\operatorname{Ind}^{G}(X, A ; \mathbb{K}) \cap \check{H}^{p}(B G ; \mathbb{K})
$$

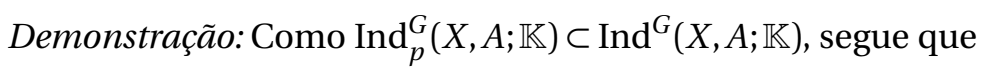

$$
\bigoplus_{p \geq 0} \operatorname{Ind}_{p}^{G}(X, A ; \mathbb{K})
$$

está contido em $\operatorname{Ind}^{G}(X, A ; \mathbb{K})$. Reciprocamente, dado

$$
\lambda=\Sigma \lambda_{p} \in \operatorname{Ind}^{G}(X, A ; \mathbb{K}), \text { então } \lambda x=\Sigma_{p, q}\left(\check{q}_{X}^{*}\left(\lambda_{p}\right) \smile x_{q}\right)=0,
$$

o que implica $\lambda_{p} x_{q}=\check{q}_{X}^{*}\left(\lambda_{p}\right) \smile x_{q}=0$, ou seja, $\lambda_{p} \in \operatorname{Ind}_{p}^{G}(X, A ; \mathbb{K})$ e, portanto, $\operatorname{Ind}^{G}(X, A ; \mathbb{K})$ está contido em $\bigoplus_{p \geq 0} \operatorname{Ind}_{p}^{G}(X, A ; \mathbb{K})$.

A seguir, apresentaremos as propriedades do $G$-índice de Fadell e Husseini.

teorema 3.2.5. Propriedade da Monotocidade Forte.

Seja $f:(X, A) \rightarrow(Y, B)$ uma aplicação equivariante entre $G$-pares paracompactos. Se o homomorfismo $f_{G}^{*}: H_{G}^{*}(Y, B ; \mathbb{K}) \rightarrow H_{G}^{*}(X, A ; \mathbb{K})$ for sobrejetor, então

$$
\operatorname{Ind}^{G}(Y, B ; \mathbb{K}) \subset \operatorname{Ind}^{G}(X, A ; \mathbb{K}) .
$$

Demonstração: Sejam $\lambda \in \operatorname{Ind}^{G}(Y, B ; \mathbb{K})$ e $u \in H_{G}^{*}(X, A, \mathbb{K})$. Como $f_{G}^{*}$ é sobrejetor, existe $v \in H_{G}^{*}(Y, B, \mathbb{K})$ tal que $f_{G}^{*}(v)=u$. Logo,

$$
\lambda u=\lambda f_{G}^{*}(v)=f_{G}^{*}(\lambda v)=f_{G}^{*}(0)=0,
$$

ou seja, $\lambda \in \operatorname{An} H_{G}^{*}(X, A ; \mathbb{K})=\operatorname{Ind}^{G}(X, A ; \mathbb{K})$. Portanto,

$$
\operatorname{Ind}^{G}(Y, B ; \mathbb{K}) \subset \operatorname{Ind}^{G}(X, A ; \mathbb{K}) .
$$

Corolario 3.2.6. Seja $f:(X, A) \rightarrow(Y, B)$ uma aplicação equivariante entre G-pares paracompactos. Se o homomorfismo

$$
f_{G}^{*}: H_{G}^{*}(Y, B, \mathbb{K}) \rightarrow H_{G}^{*}(X, A, \mathbb{K})
$$

for sobrejetor, então

$$
\left|\operatorname{Ind}^{G}(X, A ; \mathbb{K})\right| \leq\left|\operatorname{Ind}^{G}(Y, B ; \mathbb{K})\right| .
$$

Demonstração: Segue do Teorema 3.2.5 que $\operatorname{Ind}^{G}(Y, B ; \mathbb{K}) \subset \operatorname{Ind}^{G}(X, A ; \mathbb{K})$, dessa forma, está bem definido um $\mathbb{K}$-homomorfismo:

$$
\phi: \frac{\check{H}^{*}(B G ; \mathbb{K})}{\operatorname{Ind}^{G}(Y, B ; \mathbb{K})} \rightarrow \frac{\check{H}^{*}(B G ; \mathbb{K})}{\operatorname{Ind}^{G}(X, A ; \mathbb{K})}
$$


dado por $\lambda+\operatorname{Ind}^{G}(Y, B ; \mathbb{K}) \mapsto \lambda+\operatorname{Ind}^{G}(X, A ; \mathbb{K})$. Como o homomorfismo $\phi$ é sobrejetor, segue do Teorema da Dimensão ${ }^{1}$ que

$$
\operatorname{dim}_{\mathbb{K}}\left[\frac{\check{H}^{*}(B G ; \mathbb{K})}{\operatorname{Ind}^{G}(X, A ; \mathbb{K})}\right]+\operatorname{dim}_{\mathbb{K}} \operatorname{ker} \phi=\operatorname{dim}_{\mathbb{K}}\left[\frac{\check{H}^{*}(B G ; \mathbb{K})}{\operatorname{Ind}^{G}(Y, B ; \mathbb{K})}\right]
$$

e, portanto, $\left|\operatorname{Ind}^{G}(X, A ; \mathbb{K})\right| \leq\left|\operatorname{Ind}^{G}(Y, B ; \mathbb{K})\right|$.

teorema 3.2.7. Propriedade do Piercing Forte.

Sejam $(X, A)$ um $G$-par paracompacto, $Y=X \times I$ com a $G$-ação diagonal e $Z=A \times I$, onde $I=[0,1]$ é munido da $G$-ação trivial. Denotemos por $\left(X_{t}, A_{t}\right)=(X, A) \times\{t\}, t \in I$. Suponhamos que

$$
Y=X \times I=Y_{0} \cup Y_{1}, \quad X_{0} \subset Y_{0} \quad e \quad X_{1} \subset Y_{1},
$$

onde $Y_{0}$ e $Y_{1}$ são subconjuntos fechados $G$-invariantes de $Y$. Então, se $Z_{i}=Z \cap Y_{i}, i=0,1$, temos que

$$
\operatorname{Ind}^{G}\left(Y_{0} \cap Y_{1}, Z_{0} \cap Z_{1} ; \mathbb{K}\right) \subset \operatorname{Ind}^{G}(X, A ; \mathbb{K})
$$

Demonstração: Consideremos as inclusões naturais:
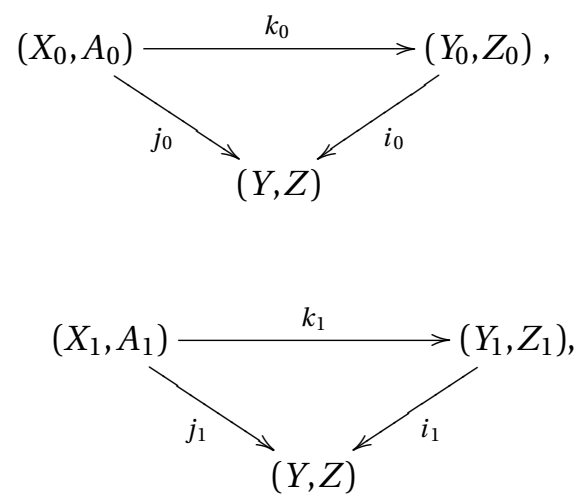

$$
\ell_{0}:\left(Y_{0} \cap Y_{1}, Z_{0} \cap Z_{1}\right) \hookrightarrow\left(Y_{0}, Z_{0}\right) \quad e \quad \ell_{1}:\left(Y_{0} \cap Y_{1}, Z_{0} \cap Z_{1}\right) \hookrightarrow\left(Y_{1}, Z_{1}\right),
$$

as quais induzem $\check{H}^{*}(B G ; \mathbb{K})$-homomorfismos sobre a cohomologia $G$-equivariante de Borel. Agora, consideremos a seqüência de Mayer-Vietoris

$$
\cdots \rightarrow H_{G}^{q}(Y, Z ; \mathbb{K}) \stackrel{\zeta}{\rightarrow} H_{G}^{q}\left(Y_{0}, Z_{0} ; \mathbb{K}\right) \oplus H_{G}^{q}\left(Y_{1}, Z_{1} ; \mathbb{K}\right) \stackrel{\eta}{\rightarrow} H_{G}^{q}\left(Y_{0} \cap Y_{1}, Z_{0} \cap Z_{1} ; \mathbb{K}\right) \rightarrow \cdots
$$

onde $\zeta=\left(i_{0 G}^{*},-i_{1 G}^{*}\right), \eta=\ell_{0 G}^{*}+\ell_{1 G}^{*}$. Mostremos inicialmente que $\ell_{0 G}^{*}$ é injetora. Com efeito, seja $x \in H_{G}^{q}\left(Y_{0}, Z_{0} ; \mathbb{K}\right)$ tal que $\ell_{0 G}^{*}(x)=0$. Então, $\eta(x, 0)=\ell_{0 G}^{*}(x)+\ell_{1 G}^{*}(0)=0 \mathrm{e}$, segue da exatidão da sequência de Mayer-Vietoris, que existe $y \in H_{G}^{q}(Y, Z ; \mathbb{K})$ tal que

$$
\zeta(y)=\left(i_{0 G}^{*}(y),-i_{1 G}^{*}(y)\right)=(x, 0) .
$$

\footnotetext{
${ }^{1}$ [29, Corolário 2.14, pg. 187] Se $f: V \rightarrow W$ é um homomorfismo de espaços vetoriais então $\operatorname{dim} \operatorname{Im} f+$ $\operatorname{dim} \operatorname{ker} f=\operatorname{dim} V$
} 
Assim, $i_{1 G}^{*}(y)=0$ e usando o diagrama comutativo (3.11), temos que

$$
k_{1 G}^{*} i_{1 G}^{*}=j_{1 G}^{*} .
$$

Desde que $j_{1}:\left(X_{1}, A_{1}\right) \hookrightarrow(Y, Z)$ é uma equivalência de homotopia, segue que $j_{1 G}^{*}$ é um isomorfismo e, deste modo, $y=0$. Assim, $x=i_{0 G}^{*}(y)=i_{0 G}^{*}(0)=0$, ou seja, $\ell_{0 G}^{*}$ é injetora. Agora, seja $\gamma:\left(Y_{0}, Z_{0}\right) \rightarrow\left(X_{0}, A_{0}\right)$ a projeção $\gamma(x, t)=(x, 0)$, a qual é $G$-equivariante, desde que,

$$
\begin{aligned}
\gamma(g(x, t)) & =\gamma(g x, t) \\
& =(g x, 0) \\
& =g(x, 0) \\
& =g \gamma(x, t), \forall g \in G, \forall(x, t) \in Y_{0} .
\end{aligned}
$$

Como

$$
\gamma k_{0}(x, 0)=\gamma(x, 0)=(x, 0)=\operatorname{Id}(x, 0),
$$

segue que $k_{0 G}^{*} \gamma_{G}^{*}=$ Id, ou seja, $\gamma_{G}^{*}$ é injetora. Denotemos $\gamma_{01}=\gamma \ell_{0}$ e, portanto, $\gamma_{01 G}^{*}=$ $\ell_{0 G}^{*} \gamma_{G}^{*}$. Em outras palavras, temos o seguinte diagrama comutativo

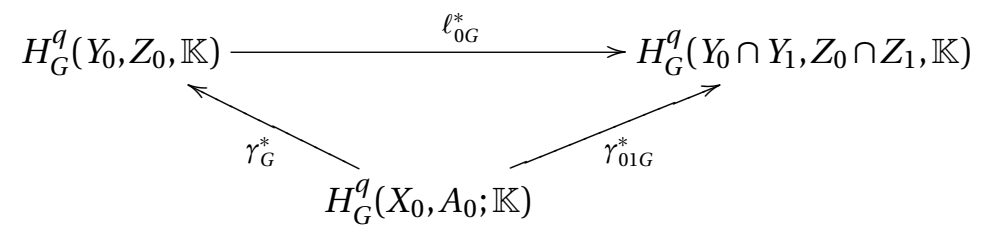

Como $\ell_{0 G}^{*}$ e $\gamma_{G}^{*}$ são injetoras, temos que $\gamma_{01 G}^{*}$ é injetora. Agora, sejam

$$
\lambda \in \operatorname{Ind}^{G}\left(Y_{0} \cap Y_{1}, Z_{0} \cap Z_{1} ; \mathbb{K}\right)
$$

e $u \in H_{G}^{*}\left(X_{0}, A_{0} ; \mathbb{K}\right)$. Desde que $\gamma_{01 G}^{*}$ é um homomorfismo de $\check{H}^{*}(B G ; \mathbb{K})$-módulos,

$$
\gamma_{01 G}^{*}(\lambda u)=\lambda \gamma_{01 G}^{*}(u)=0
$$

e, como $\gamma_{01 G}^{*}$ é injetora, concluímos que $\lambda u=0$. Portanto,

$$
\operatorname{Ind}^{G}\left(Y_{0} \cap Y_{1}, Z_{0} \cap Z_{1} ; \mathbb{K}\right) \subset \operatorname{Ind}^{G}(X, A ; \mathbb{K}) .
$$

teorema 3.2.8. Propriedade da Continuidade.

Suponhamos que $H_{G}^{*}(X, A ; \mathbb{K})$ seja finitamente gerado sobre $\check{H}^{*}(B G ; \mathbb{K})$ e seja $(X, A)$ um $G$-par paracompacto, onde $X$ é um subconjunto fechado $G$-invariante do $G$-espaço paracompacto $Y$. Então, dadas vizinhanças $G$-invariantes em $Y$ : $U$ e $V$ de $X$ e A, respectivamente, existem vizinhanças $G$-invariantes fechadas $N$ e $M, M \subset N$, de $X$ e A, respectivamente, tais que $N \subset U, M \subset V e$

$$
\operatorname{Ind}^{G}(N, M ; \mathbb{K}) \subset \operatorname{Ind}^{G}(X, A ; \mathbb{K}) .
$$


Demonstração: Seja $\mathscr{P}$ a família de todos os pares de vizinhanças $G$-invariantes fechadas $(N, M)$. Segue do Teorema da Continuidade na Cohomologia Equivariante de Borel (Teorema 3.1.7) que

$$
\begin{aligned}
H_{G}^{*}(X, A ; \mathbb{K}) & \cong \lim _{\substack{\overrightarrow{(N, M) \in \mathscr{P}}\\
}} H_{G}^{*}(N, M ; \mathbb{K}) \\
& \cong \frac{\bigoplus_{(N, M) \in \mathscr{P}} H_{G}^{*}(N, M ; \mathbb{K})}{<S>}
\end{aligned}
$$

Desde que $H_{G}^{*}(X, A ; \mathbb{K})$ é finitamente gerado sobre $\check{H}^{*}(B G ; \mathbb{K})$, sejam $x^{1}, \ldots, x^{r}$ os geradores deste $\breve{H}^{*}(B G ; \mathbb{K})$-módulo, e segue da Observação 1.1.5 que existem pares

$$
\left(N_{1}, M_{1}\right), \ldots,\left(N_{r}, M_{r}\right) \in \mathscr{P}
$$

tais que

$$
x^{i}=x_{\left(N_{i}, M_{i}\right)}^{i}+\langle S\rangle, \operatorname{com} x_{\left(N_{i}, M_{i}\right)}^{i} \in H_{G}^{*}\left(N_{i}, M_{i} ; \mathbb{K}\right) \text {, para cada } i=1, \ldots, r .
$$

Como $\mathscr{P}$ é um conjunto dirigido, existe um $\operatorname{par}(N, M) \in \mathscr{P}$ tal que

$$
\left(N_{i}, M_{i}\right) \leq(N, M), \forall i=1, \ldots, r
$$

e, assim

$$
x^{i}=x_{\left(N_{i}, N_{i}\right)}^{i}+\langle S\rangle=x_{(N, M)}^{i}+\langle S\rangle, \operatorname{com} x_{(N, M)}^{i} \in H_{G}^{*}(N, M ; \mathbb{K}), i=1, \ldots, r .
$$

Deste modo, o $\check{H}^{*}(B G ; \mathbb{K})$-homomorfismo natural

$$
\begin{aligned}
H_{G}^{*}(N, M ; \mathbb{K}) & \rightarrow H_{G}^{*}(X, A ; \mathbb{K}) \\
x_{(N, N)} & \mapsto x_{(N, N)}+\langle S\rangle
\end{aligned}
$$

é sobrejetor e, segue do Teorema 3.2.5 (Monotocidade Forte) que

$$
\operatorname{Ind}^{G}(N, M ; \mathbb{K}) \subset \operatorname{Ind}^{G}(X, A ; \mathbb{K})
$$

\subsubsection{G-índice absoluto}

Nesta seção, estudaremos o caso absoluto da Definição 3.2.2 (ou seja, $A=\emptyset$ ). Denotaremos o $G$-índice cohomológico de Fadell e Husseini com valor ideal de $X$ por $\operatorname{Ind}^{G}(X ; \mathbb{K})$. Neste caso, temos incialmente o seguinte

TEOREMA 3.2.9. Se X for um $G$-espaço paracompacto, então

$$
\operatorname{Ind}^{G}(X ; \mathbb{K})=\operatorname{ker}\left(q_{X}^{*}: \check{H}^{*}(B G ; \mathbb{K}) \rightarrow H_{G}^{*}(X ; \mathbb{K})\right) .
$$


Demonstração: Se $\lambda \in \operatorname{Ind}^{G}(X, \mathbb{K})$, considerando $x=1 \in H_{G}^{*}(X, \mathbb{K})$ temos

$$
0=\lambda 1=q_{X}^{*}(\lambda) \smile 1=q_{X}^{*}(\lambda)
$$

e, portanto, $\lambda \in \operatorname{ker} q_{G}^{*}$. Reciprocamente, se $\lambda \in \operatorname{ker} q_{X}^{*}$, temos que

$$
\lambda x=q_{X}^{*}(\lambda) \smile x=0 \smile x=0, \text { para todo } x \in H_{G}^{*}(X, \mathbb{K}) .
$$

Portanto, $\lambda \in \operatorname{Ind}^{G}(X ; \mathbb{K})$.

Como uma consequência, temos o seguinte

COROLARIO 3.2.10. Se X for um G-espaço paracompacto, então o G-índice cohomológico de Fadell e Husseini com valor numérico é dado por

$$
\left|\operatorname{Ind}^{G}(X ; \mathbb{K})\right|=\operatorname{dim}_{\mathbb{K}}\left[\frac{\check{H}^{*}(B G ; \mathbb{K})}{\operatorname{ker} q_{X}^{*}}\right]=\operatorname{dim}_{\mathbb{K}} \operatorname{Im} q_{X}^{*}
$$

Demonstração: Segue do Teorema do Isomorfismo para a aplicação

$$
q_{X}^{*}: \check{H}^{*}(B G ; \mathbb{K}) \rightarrow H_{G}^{*}(X ; \mathbb{K}), \text { que } \operatorname{dim}_{\mathbb{K}}\left[\frac{\check{H}^{*}(B G ; \mathbb{K})}{\operatorname{ker} q_{X}^{*}}\right]=\operatorname{dim}_{\mathbb{K}} \operatorname{Im} q_{X}^{*}
$$

Logo, substituindo $\operatorname{Ind}^{G}(X ; \mathbb{K})=\operatorname{ker} q_{X}^{*}$ (Teorema 3.2.9) e, usando a definição do $G$-indice de valor numérico (Definição 3.2.3), obtemos o resultado.

No caso em que $X$ é um $G$-espaço livre, temos o seguinte

COROLARIO 3.2.11. Se X for um G-espaço paracompacto com G -ação livre,

$$
\operatorname{Ind}^{G}(X ; \mathbb{K})=\operatorname{ker}\left(q_{G}^{*}: \check{H}^{*}(B G ; \mathbb{K}) \rightarrow \check{H}^{*}(X / G ; \mathbb{K})\right),
$$

onde $q_{G}: X / G \rightarrow B G$ é uma aplicação classificante para o $G$ fibrado principal $X \rightarrow X / G$.

Demonstração: Segue da Proposição 3.1.6 que $H_{G}^{*}\left(X_{G} ; \mathbb{K}\right) \cong \check{H}^{*}(X / G ; \mathbb{K})$. Portanto, do Teorema 3.2.9 concluímos que

$$
\operatorname{Ind}^{G}(X ; \mathbb{K})=\operatorname{ker}\left(q_{G}^{*}: \check{H}^{*}(B G ; \mathbb{K}) \rightarrow H_{G}^{*}(X ; \mathbb{K}) \cong \check{H}^{*}(X / G ; \mathbb{K})\right) .
$$

teorema 3.2.12. Propriedade da Monotocidade Fraca.

Se $f: X \rightarrow Y$ for uma aplicação $G$-equivariante entre $G$-espaços paracompactos, então

$$
\operatorname{Ind}^{G}(Y ; \mathbb{K}) \subset \operatorname{Ind}^{G}(X ; \mathbb{K}) .
$$

Demonstração: Da observação 3.1.17 tem-se que $q_{X}^{*}=\left(q_{Y} f_{G}\right)^{*}=f_{G}^{*} q_{Y}^{*}$. Deste modo, se $\lambda \in \operatorname{Ind}^{G}(Y ; \mathbb{K})=\operatorname{ker} q_{Y}^{*}$, então $q_{Y}^{*}(\lambda)=0$. Assim,

$$
q_{X}^{*}(\lambda)=f_{G}^{*}\left(q_{Y}^{*}(\lambda)\right)=0 \text {, ou seja, } \lambda \in \operatorname{ker} q_{X}^{*}=\operatorname{Ind}^{G}(X ; \mathbb{K}) .
$$

Portanto,

$$
\operatorname{Ind}^{G}(Y ; \mathbb{K}) \subset \operatorname{Ind}^{G}(X ; \mathbb{K})
$$


COROLARIO 3.2.13. Se $f: X \rightarrow Y$ for uma aplicação $G$-equivariante entre $G$-espaços paracompactos, então

$$
\left|\operatorname{Ind}^{G}(X ; \mathbb{K})\right| \leq\left|\operatorname{Ind}^{G}(Y ; \mathbb{K})\right| .
$$

Demonstração: Desde que $\operatorname{Ind}^{G}(Y ; \mathbb{K}) \subset \operatorname{Ind}^{G}(X ; \mathbb{K})$ (Teorema 3.2.12), a demonstração é análoga à demonstração do Corolário 3.2.6.

COROLARIo 3.2.14. Se $f: X \rightarrow Y$ for um homeomorfismo equivariante de G-espaços paracompactos, então

$$
\operatorname{Ind}^{G}(X ; \mathbb{K})=\operatorname{Ind}^{G}(Y ; \mathbb{K})
$$

Demonstração: Desde que $f: X \rightarrow Y$ é um homeomorfismo $G$-equivariante, existe $\tilde{f}: Y \rightarrow X$ inversa $G$-equivariante. Segue do Teorema 3.2.12 que

$$
\operatorname{Ind}^{G}(X ; \mathbb{K}) \subset \operatorname{Ind}^{G}(Y ; \mathbb{K}) \quad e \quad \operatorname{Ind}^{G}(X ; \mathbb{K}) \supset \operatorname{Ind}^{G}(Y ; \mathbb{K}) .
$$

PROPOSIÇão 3.2.15. Sejam $X_{1}$ e $X_{2} G$-espaços paracompactos tais que o $G$-espaço produto $X_{1} \times X_{2}$ com ação diagonal seja paracompacto. Então,

$$
\operatorname{Ind}^{G}\left(X_{1} \times X_{2} ; \mathbb{K}\right) \supset \operatorname{Ind}^{G}\left(X_{1} ; \mathbb{K}\right) \cap \operatorname{Ind}^{G}\left(X_{2} ; \mathbb{K}\right)
$$

Demonstração: Consideremos a $i$-ésima projeção $\pi_{i}: X_{1} \times X_{2} \rightarrow X_{i}$ para $i=1,2$, as quais são $G$-equivariantes. Então, segue do Teorema 3.2.12 que

$$
\operatorname{Ind}^{G}\left(X_{1} \times X_{2} ; \mathbb{K}\right) \supset \operatorname{Ind}^{G}\left(X_{i} ; \mathbb{K}\right), \quad i=1,2 .
$$

Portanto,

$$
\operatorname{Ind}^{G}\left(X_{1} \times X_{2} ; \mathbb{K}\right) \supset \operatorname{Ind}^{G}\left(X_{1} ; \mathbb{K}\right) \cap \operatorname{Ind}^{G}\left(X_{2} ; \mathbb{K}\right) .
$$

PRoposição 3.2.16. Sejam $X_{1}$ e $X_{2} G$-espaços paracompactos tais que o G-espaço join $X_{1} *$ $X_{2}$ com ação diagonal seja paracompacto. Então,

$$
\operatorname{Ind}^{G}\left(X_{1} * X_{2} ; \mathbb{K}\right) \subset \operatorname{Ind}^{G}\left(X_{1} ; \mathbb{K}\right)+\operatorname{Ind}^{G}\left(X_{2} ; \mathbb{K}\right)
$$

Demonstração: Consideremos os mergulhos $X_{i} \rightarrow X_{1} * X_{2}$ para $i=1,2$, as quais são $G$ equivariantes. Então, segue do Teorema 3.2.12 que

$$
\operatorname{Ind}^{G}\left(X_{1} * X_{2} ; \mathbb{K}\right) \subset \operatorname{Ind}^{G}\left(X_{i} ; \mathbb{K}\right), \quad i=1,2 .
$$

Portanto,

$$
\operatorname{Ind}^{G}\left(X_{1} * X_{2} ; \mathbb{K}\right) \subset \operatorname{Ind}^{G}\left(X_{1} ; \mathbb{K}\right)+\operatorname{Ind}^{G}\left(X_{2} ; \mathbb{K}\right) .
$$

teorema 3.2.17. Propriedade da Aditividade.

Seja $X=X_{1} \cup X_{2}$, onde $X, X_{1}$ e $X_{2}$ são $G$-espaços paracompactose $\left\{X_{1}, X_{2}\right\}$ é um par excisivo de X. Então,

$$
\operatorname{Ind}^{G}\left(X_{1} ; \mathbb{K}\right) \cdot \operatorname{Ind}^{G}\left(X_{2} ; \mathbb{K}\right) \subset \operatorname{Ind}^{G}(X ; \mathbb{K}),
$$

onde $\cdot$ é o produto de ideais. 
Demonstração: Sejam $\lambda=\Sigma \lambda_{p} \in \operatorname{Ind}^{G}\left(X_{1} ; \mathbb{K}\right)$ e $v=\Sigma v_{p} \in \operatorname{Ind}^{G}\left(X_{2} ; \mathbb{K}\right)$. Mostremos que, para todo $x=\Sigma x_{q} \in H_{G}^{*}(X ; \mathbb{K})$,

$$
(\lambda \smile v) x=\lambda(v x)=q_{X}^{*}(\lambda) \smile v x=0
$$

Pelo Axioma da Exatidão na Cohomologia $G$-equivariante de Borel (Proposição 3.1.4), a seguinte sequência longa é exata

$$
\cdots \stackrel{\Delta}{\longrightarrow} H_{G}^{p}\left(X, X_{1} ; \mathbb{K}\right) \stackrel{j_{1 G}^{*}}{\longrightarrow} H_{G}^{p}(X ; \mathbb{K}) \stackrel{\left.i_{X}^{*}\right|_{\check{H}^{p}}\left(B G ; \mathbb{K}_{X_{1}}^{*}\right.}{\longrightarrow} H_{G}^{p}\left(X_{1}, \mathbb{K}\right) \stackrel{\Delta}{\longrightarrow} \cdots
$$

Desde que, da Proposição 2.3.13, o triângulo do diagrama acima é comutativo e $\operatorname{Ind}^{G}\left(X_{1} ; \mathbb{K}\right)=\operatorname{ker} q_{X_{1}}^{*}$, temos que

$$
i_{1 G}^{*}\left(q_{X}^{*}\left(\lambda_{p}\right)\right)=q_{X_{1}}^{*}\left(\lambda_{p}\right)=0
$$

e, deste modo, $q_{X}^{*}\left(\lambda_{p}\right) \in \operatorname{ker} i_{1 G}^{*}$. Segue da exatidão da sequência (3.18), que existe $\beta_{1}^{p} \in$ $H_{G}^{p}\left(X, X_{1} ; \mathbb{K}\right)$ tal que

$$
j_{1 G}^{*}\left(\beta_{1}^{p}\right)=q_{X}^{*}\left(\lambda_{p}\right) .
$$

Assim, existe $\beta_{1} \in H_{G}^{*}\left(X, X_{1} ; \mathbb{K}\right)$ tal que

$$
j_{1 G}^{*}\left(\beta_{1}\right)=q_{X}^{*}(\lambda) .
$$

Considerando novamente o Axioma da Exatidão, temos que a seguinte sequência longa também é exata

$$
\cdots \stackrel{\Delta}{\longrightarrow} H_{G}^{l+q}\left(X, X_{2} ; \mathbb{K}\right) \stackrel{J_{2 G}^{*}}{\longrightarrow} H_{G}^{l+q}(X ; \mathbb{K}) \stackrel{l_{2 G}^{*}}{\longrightarrow} H_{G}^{l+q}\left(X_{2} ; \mathbb{K}\right) \stackrel{\Delta}{\longrightarrow} \cdots .
$$

Como $\imath_{2 G}^{*}$ é um homomorfismo de $H^{*}(B G ; \mathbb{K})$-módulos graduados, $v_{l} \in \operatorname{Ind}_{l}^{G}\left(X_{2} ; \mathbb{K}\right)$ e $i_{2 G}^{*}\left(x_{q}\right) \in H_{G}^{q}\left(X_{2} ; \mathbb{K}\right)$ temos que

$$
i_{2 G}^{*}\left(v_{l} x_{q}\right)=v_{l} i_{2 G}^{*}\left(x_{q}\right)=0 \text {, ou seja, } v_{l} x_{q} \in \operatorname{ker} i_{2 G}^{*} .
$$

Segue da exatidão da sequência (3.20), que existe $\beta_{2}^{l+q} \in H_{G}^{l+q}\left(X, X_{2} ; \mathbb{K}\right)$ tal que

$$
j_{2 G}^{*}\left(\beta_{2}^{l+q}\right)=v_{l} x_{q}
$$

e, deste modo, existe $\beta_{2} \in H_{G}^{*}\left(X, X_{2} ; \mathbb{K}\right)$ tal que

$$
j_{2 G}^{*}\left(\beta_{2}\right)=v x .
$$

Substituindo as equações (3.19) e (3.21) na equação (3.17) e usando a Proposição 3.1.8 e a observação 3.1.18 temos que

$$
(\lambda \smile v) x=j_{1 G}^{*}\left(\beta_{1}\right) \smile j_{2 G}^{*}\left(\beta_{2}\right)=j_{G}^{*}\left(\beta_{1} \smile \beta_{2}\right),
$$

onde $j_{G}^{*}$ é a induzida da iclusão $j: X \rightarrow\left(X, X_{1} \cup X_{2}\right)$. Como $\left\{X_{1}, X_{2}\right\}$ é um par excisivo de $X$, segue que $\beta_{1} \smile \beta_{2} \in H_{G}^{*}(X, X ; \mathbb{K})=0 \mathrm{e}$, assim, $(\lambda \smile v) x=0$. Portanto,

$$
\operatorname{Ind}^{G}\left(X_{1} ; \mathbb{K}\right) \cdot \operatorname{Ind}^{G}\left(X_{2} ; \mathbb{K}\right) \subset \operatorname{Ind}^{G}(X ; \mathbb{K}) .
$$


Corolario 3.2.18. Seja $X=X_{1} \cup X_{2}$, onde $X, X_{1}$ e $X_{2} G$-espaços paracompactos e $\left\{X_{1}, X_{2}\right\}$ é um par excisivo de $X$. Então,

$$
\left|\operatorname{Ind}^{G}(X ; \mathbb{K})\right| \leq\left|\operatorname{Ind}^{G}\left(X_{1} ; \mathbb{K}\right)\right|+\left|\operatorname{Ind}^{G}\left(X_{2} ; \mathbb{K}\right)\right| .
$$

Demonstração: Definimos o homomorfismo sobrejetor

$$
\begin{gathered}
\kappa: \frac{\check{H}^{*}(B G ; \mathbb{K})}{\operatorname{Ind}^{G}\left(X_{1} ; \mathbb{K}\right)} \times \frac{\check{H} *(B G ; \mathbb{K})}{\operatorname{Ind}^{G}\left(X_{2} ; \mathbb{K}\right)} \longrightarrow \frac{\check{H}^{*}(B G ; \mathbb{K})}{\operatorname{Ind}^{G}\left(X_{1} ; \mathbb{K}\right) \cdot \operatorname{Ind}^{G}\left(X_{2} ; \mathbb{K}\right)} \\
\kappa\left(\lambda_{1}+\operatorname{Ind}^{G}\left(X_{1} ; \mathbb{K}\right), \lambda_{2}+\operatorname{Ind}^{G}\left(X_{2} ; \mathbb{K}\right)\right)=\left(\lambda_{1}+\lambda_{2}\right)+\operatorname{Ind}^{G}\left(X_{1} ; \mathbb{K}\right) \cdot \operatorname{Ind}^{G}\left(X_{2} ; \mathbb{K}\right) .
\end{gathered}
$$

Do Teorema 3.2.17, está bem definido um homomorfismo sobrejetor

$$
\psi: \frac{\check{H}^{*}(B G ; \mathbb{K})}{\operatorname{Ind}^{G}\left(X_{1} ; \mathbb{K}\right) \cdot \operatorname{Ind}^{G}\left(X_{2} ; \mathbb{K}\right)} \longrightarrow \frac{\check{H}^{*}(B G ; \mathbb{K})}{\operatorname{Ind}^{G}(X ; \mathbb{K})}
$$

definido pela expressão

$$
\psi\left(\lambda+\operatorname{Ind}^{G}\left(X_{1} ; \mathbb{K}\right) \cdot \operatorname{Ind}^{G}\left(X_{2} ; \mathbb{K}\right)\right)=\lambda+\operatorname{Ind}^{G}(X ; \mathbb{K})
$$

Desde que $\psi \kappa$ é sobrejor e

$$
\operatorname{dim}_{\mathbb{K}}\left[\frac{\check{H}^{*}(B G ; \mathbb{K})}{\operatorname{Ind}^{G}\left(X_{1} ; \mathbb{K}\right)} \times \frac{\check{H}^{*}(B G ; \mathbb{K})}{\operatorname{Ind}^{G}\left(X_{2} ; \mathbb{K}\right)}\right]=\operatorname{dim}_{\mathbb{K}}\left[\frac{\check{H}^{*}(B G ; \mathbb{K})}{\operatorname{Ind}^{G}\left(X_{1} ; \mathbb{K}\right)}\right]+\operatorname{dim}_{\mathbb{K}}\left[\frac{\check{H}^{*}(B G ; \mathbb{K})}{\operatorname{Ind}^{G}\left(X_{2} ; \mathbb{K}\right)}\right],
$$

segue que

$$
\left|\operatorname{Ind}^{G}(X ; \mathbb{K})\right| \leq\left|\operatorname{Ind}^{G}\left(X_{1} ; \mathbb{K}\right)\right|+\left|\operatorname{Ind}^{G}\left(X_{2}, \mathbb{K}\right)\right| .
$$

teorema 3.2.19. Propriedade Piercing Fraco.

Seja $X$ um $G$-espaço paracompacto, $Y=X \times I$ com a $G$-ação diagonal, onde $I=[0,1]$ é munido da $G$-ação trivial. Denotemos por $X_{t}=X \times\{t\}, t \in I$. Suponhamos que

$$
Y=X \times I=Y_{0} \cup Y_{1}, \quad X_{0} \subset Y_{0} \quad e \quad X_{1} \subset Y_{1}
$$

onde $Y_{0}$ e $Y_{1}$ são subconjuntos $G$-invariantes fechados de $Y$. Então

$$
\operatorname{Ind}^{G}\left(Y_{0} \cap Y_{1} ; \mathbb{K}\right)=\operatorname{Ind}^{G}(X ; \mathbb{K}) .
$$

Demonstração: Segue do Teorema 3.2.7, para $A=\emptyset$, que

$$
\operatorname{Ind}^{G}\left(Y_{0} \cap Y_{1} ; \mathbb{K}\right) \subset \operatorname{Ind}^{G}(X ; \mathbb{K}) .
$$

Agora, seja $\gamma: Y_{0} \cap Y_{1} \rightarrow X_{0}$ a projeção $\gamma(x, t)=(x, 0)$, a qual é $G$-equivariante. Então, segue do Teorema 3.2.12

$$
\operatorname{Ind}^{G}(X ; \mathbb{K})=\operatorname{Ind}^{G}\left(X_{0} ; \mathbb{K}\right) \subset \operatorname{Ind}^{G}\left(Y_{0} \cap Y_{1} ; \mathbb{K}\right) .
$$




\subsection{G-índice do Produto e do Join}

Nesta seção, apresentamos os principais resultados que descrevem o $G$-índice de Fadell e Husseini com valor ideal dos espaços produto e join, em função dos $G$-índices dos espaços fatores, no caso em que o grupo $G$ também é um grupo produto.

Sejam $X_{i}$ um $G_{i}$-espaço paracompacto, $i=1,2$, com $G=G_{1} \times G_{2}$ tal que o $G$-espaço produto $X=X_{1} \times X_{2}$ também seja paracompacto. Consideremos as aplicações classificantes para os grupos compactos de Lie $G_{1}, G_{2}$ e $G$

$$
\begin{gathered}
q_{X_{1}}: E G_{1} \times_{G_{1}} X_{1} \rightarrow B G_{1}, \quad q_{X_{2}}: E G_{2} \times_{G_{2}} X_{2} \rightarrow B G_{2}, \\
q_{X}: E G \times_{G} X \rightarrow B G .
\end{gathered}
$$

Fazendo uma ordenação dos elementos, obtemos a seguinte identificação

$$
\left(E G_{1} \times X_{1}\right) \times\left(E G_{2} \times X_{2}\right) \approx\left(E G_{1} \times E G_{2}\right) \times\left(X_{1} \times X_{2}\right) .
$$

Agora consideremos o produto das aplicações projeções

$$
\pi \times \pi:\left(E G_{1} \times X_{1}\right) \times\left(E G_{2} \times X_{2}\right) \rightarrow\left(E G_{1} \times_{G_{1}} X_{1}\right) \times\left(E G_{2} \times_{G_{2}} X_{2}\right) .
$$

Desde que

$$
\begin{aligned}
(\pi \times \pi)^{-1}\left(\left[m_{1}, x_{1}\right],\left[m_{2}, x_{2}\right]\right) & =\left\{\left(\left(g_{1} m_{1}, g_{1} x_{1}\right),\left(g_{2} m_{2}, g_{2} x_{2}\right)\right)\right\} \\
\stackrel{3.22}{=} & \left\{\left(\left(g_{1} m_{1}, g_{2} m_{2}\right),\left(g_{1} x_{1}, g_{2} x_{2}\right)\right)\right\} \\
= & \left\{\left(g_{1}, g_{2}\right)\left(\left(m_{1}, m_{2}\right),\left(x_{1}, x_{2}\right)\right)\right\} \\
& =\left[\left(\left(m_{1}, m_{2}\right),\left(x_{1}, x_{2}\right)\right)\right],
\end{aligned}
$$

então a decomposição de $\left(E G_{1} \times X_{1}\right) \times\left(E G_{2} \times X_{2}\right)$ é dada por

$$
\left\{(\pi \times \pi)^{-1}\left(\left[m_{1}, x_{1}\right],\left[m_{2}, x_{2}\right]\right)\right\}=\left(E G_{1} \times E G_{2}\right) \times_{G}\left(X_{1} \times X_{2}\right),
$$

munida da topologia quociente. Logo, usando resultados de topologia quociente, temos que a aplicação quociente $\pi \times \pi$ determina um diagrama comutativo

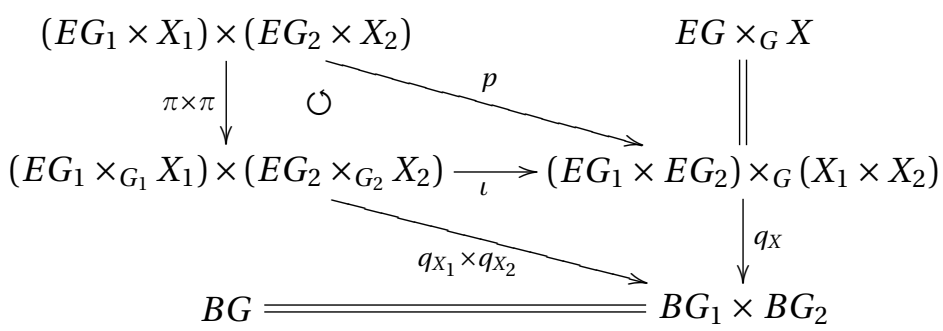

onde $\iota$ é um homeomorfismo. Sob condições apropriadas de finitude (por exemplo, $\check{H}^{*}\left(B G_{1} ; \mathbb{K}\right)$ e $H_{G_{1}}^{*}\left(X_{1} ; \mathbb{K}\right)$ são do tipo finito sobre $\left.\mathbb{K}\right)$, temos que as aplicações $q_{X}^{*}$ e $q_{X_{1}}^{*} \otimes q_{X_{2}}^{*}$ podem ser identificadas (Ver [21, pg. 75]) como mostra o seguinte diagrama

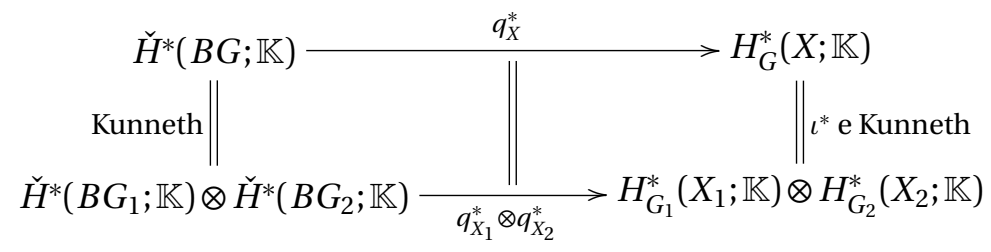


e escrevemos

$$
q_{X}^{*}(\lambda, v)=q_{X_{1}}^{*}(\lambda) \otimes q_{X_{2}}^{*}(v)
$$

Deste modo, usando esta identificação entre $q_{X}^{*}$ e $q_{X_{1}}^{*} \otimes q_{X_{2}}^{*}$, podemos enunciar os resultados a seguir.

PRoposição 3.3.1. Sejam $X_{i}$ um $G_{i}$-espaço paracompacto $, i=1,2 e, G=G_{1} \times G_{2}$ tais que $o G$-espaço produto $X=X_{1} \times X_{2}$ seja paracompacto. Então,

$$
\operatorname{Ind}^{G}(X ; \mathbb{K})=\operatorname{Ind}^{G_{1}}\left(X_{1} ; \mathbb{K}\right) \otimes \check{H}^{*}\left(B G_{2} ; \mathbb{K}\right)+\check{H}^{*}\left(B G_{1} ; \mathbb{K}\right) \otimes \operatorname{Ind}^{G_{2}}\left(X_{2} ; \mathbb{K}\right)
$$

Demonstração: Desde que $\operatorname{Ind}^{G}(X ; \mathbb{K})=\operatorname{ker} q_{X}^{*}$ (Teorema 3.2.9), dado qualquer elemento $\lambda \otimes v \in \check{H}^{*}\left(B G_{1} ; \mathbb{K}\right) \otimes \check{H}^{*}\left(B G_{2} ; \mathbb{K}\right)$ temos que

$$
\begin{aligned}
\lambda \otimes v \in \operatorname{Ind}^{G}\left(X_{1} \times X_{2} ; \mathbb{K}\right) & \Leftrightarrow q_{X_{1}}^{*}(\lambda) \otimes q_{X_{2}}^{*}(v)=0 \\
& \Leftrightarrow q_{X_{1}}^{*}(\lambda)=0 \text { ou } q_{X_{2}}^{*}(v)=0 \\
& \Leftrightarrow \lambda \in \operatorname{ker} q_{X_{1}}^{*} \text { ou } v \in \operatorname{ker} q_{X_{2}}^{*} .
\end{aligned}
$$

Portanto,

$$
\begin{gathered}
\lambda \otimes v \in \operatorname{Ind}^{G}\left(X_{1} \times X_{2} ; \mathbb{K}\right) \\
\hat{\mathbb{1}} \\
\lambda \otimes v \in \operatorname{Ind}^{G_{1}}\left(X_{1} ; \mathbb{K}\right) \otimes \check{H}^{*}\left(B G_{2} ; \mathbb{K}\right)+\check{H}^{*}\left(B G_{1} ; \mathbb{K}\right) \otimes \operatorname{Ind}^{G_{2}}\left(X_{2} ; \mathbb{K}\right) .
\end{gathered}
$$

CORolario 3.3.2. Sejam $X_{i}$ um $G_{i}$-espaço paracompacto $, i=1,2 e, G=G_{1} \times G_{2}$ tais que o $G$-espaço produto $X=X_{1} \times X_{2}$ seja paracompacto. Se

$$
\check{H}^{*}\left(B G_{1} ; \mathbb{K}\right) \cong \mathbb{K}\left[x_{1}, \ldots, x_{k}\right], \quad \check{H}^{*}\left(B G_{2} ; \mathbb{K}\right) \cong \mathbb{K}\left[y_{1}, \ldots, y_{l}\right]
$$

$e \operatorname{Ind}^{G_{1}}\left(X_{1} ; \mathbb{K}\right)=\left(f_{1}, \cdots, f_{m}\right) e \operatorname{Ind}^{G_{2}}\left(X_{2} ; \mathbb{K}\right)=\left(g_{1}, \cdots, g_{n}\right)$, onde $f_{i}$ e $g_{i}$ são polinômios em $x$ e y respectivamente, então

$$
\operatorname{Ind}^{G}\left(X_{1} \times X_{2} ; \mathbb{K}\right)=\left(f_{1}, \ldots, f_{m}, g_{1}, \ldots, g_{n}\right)
$$

o ideal gerado pelos polinômios $f_{i}$ e $g_{j}$.

Demonstração: Segundo a proposição 3.3.1, temos que

$$
\begin{aligned}
\operatorname{Ind}^{G}\left(X_{1} \times X_{2} ; \mathbb{K}\right) & =\left(f_{1}, \ldots, f_{m}\right) \otimes \mathbb{K}\left[y_{1}, \ldots, y_{l}\right]+\mathbb{K}\left[x_{1}, \ldots, x_{k}\right] \otimes\left(g_{1}, \ldots, g_{n}\right) \\
& =\left(f_{1}, \ldots, f_{m}, g_{1}, \ldots, g_{n}\right)
\end{aligned}
$$

COROLARio 3.3.3. Sejam $X_{1}$ um $G_{1}$-espaço paracompacto, $\left\{x_{0}\right\}$ um espaço topológico com $G_{2}$-ação trivial. Se $G=G_{1} \times G_{2}$, então $X_{1}$ é um $G$-espaço paracompacto e

$$
\left.\operatorname{Ind}^{G}\left(X_{1} ; \mathbb{K}\right)=\operatorname{Ind}^{G_{1}}\left(X_{1} ; \mathbb{K}\right)\right) \otimes \check{H}^{*}\left(B G_{2} ; \mathbb{K}\right)
$$

Caso análogo, temos para $X_{1}=\left\{x_{0}\right\}$ e, neste caso,

$$
\operatorname{Ind}^{G}\left(X_{2} ; \mathbb{K}\right)=\check{H}^{*}\left(B G_{1} ; \mathbb{K}\right) \otimes \operatorname{Ind}^{G_{2}}\left(X_{2} ; \mathbb{K}\right) .
$$


Demonstração: Definindo $\left(g_{1}, g_{2}\right) x_{1}=g_{1} x_{1}$, temos que $X_{1}$ é um $G$-espaço paracompacto. Desde que $X_{1}$ é homeomorfo a $X_{1} \times\left\{x_{0}\right\}$ (e portanto paracompacto), como $G$ espaços paracompactos, então pelo Corolário 3.2.14

$$
\operatorname{Ind}^{G}\left(X_{1} ; \mathbb{K}\right)=\operatorname{Ind}^{G}\left(X_{1} \times\left\{x_{0}\right\} ; \mathbb{K}\right) .
$$

Por outro lado, temos que

$$
\operatorname{Ind}^{G_{2}}\left(\left\{x_{0}\right\} ; \mathbb{K}\right)=\operatorname{ker}\left(q_{x_{0}}^{*}: \check{H}^{*}\left(B G_{2} ; \mathbb{K}\right) \rightarrow H_{G_{2}}^{*}\left(\left\{x_{0}\right\} ; \mathbb{K}\right)=\check{H}^{*}\left(B G_{2} ; \mathbb{K}\right)\right),
$$

onde $q_{x_{0}}^{*}$ é a induzida em cohomologia da aplicação classificante

$$
q_{x_{0}}:\left(\left\{x_{0}\right\} \times E G_{2}\right) / G_{2}=E G_{2} / G_{2} \rightarrow E G_{2} / G_{2},\left[x_{0}, e\right]=[e] \rightarrow[e],
$$

então $q_{x_{0}}^{*}=$ Id e, segue que,

$$
\operatorname{Ind}^{G_{2}}\left(\left\{x_{0}\right\} ; \mathbb{K}\right)=0 .
$$

Portanto, da Proposição 3.3.1 temos que

$$
\begin{aligned}
\operatorname{Ind}^{G}\left(X_{1} ; \mathbb{K}\right) & =\operatorname{Ind}^{G_{1}}\left(X_{1} ; \mathbb{K}\right) \otimes \check{H}^{*}\left(B G_{2} ; \mathbb{K}\right)+H^{*}\left(B G_{1} ; \mathbb{K}\right) \otimes 0 \\
& =\operatorname{Ind}^{G_{1}}\left(X_{1} ; \mathbb{K}\right) \otimes \check{H}^{*}\left(B G_{2} ; \mathbb{K}\right) .
\end{aligned}
$$

PRoposição 3.3.4. Sejam $X_{1}$ um $G_{1}$-espaço paracompacto, $X_{2}$ um $G_{2}$-espaço paracompacto e $G=G_{1} \times G_{2}$ tais que o $G$-espaço join $X_{1} * X_{2}$ seja paracompacto. Então,

$$
\operatorname{Ind}^{G}\left(X_{1} * X_{2} ; \mathbb{K}\right) \subset\left[\operatorname{Ind}^{G_{1}}\left(X_{1} ; \mathbb{K}\right) \otimes \check{H}^{*}\left(B G_{2} ; \mathbb{K}\right)\right] \cap\left[\check{H}^{*}\left(B G_{1} ; \mathbb{K}\right) \otimes \operatorname{Ind}^{G_{2}}\left(X_{2} ; \mathbb{K}\right)\right],
$$

ondeก representa a interseção de ideais.

Demonstração: Definindo $\left(g_{1}, g_{2}\right) x_{1}=g_{1} x_{1}$ e $\left(g_{1}, g_{2}\right) x_{2}=g_{2} x_{2}$ temos que $X_{1}$ e $X_{2}$ são $G$-espaços paracompactos. Consideremos as aplicações inclusões $G$-equivariantes

$$
\iota: X_{1} \rightarrow X_{1} * X_{2} \quad \text { e } \quad J: X_{2} \rightarrow X_{1} * X_{2} .
$$

Segue do Teorema 3.2.12 que

$$
\begin{aligned}
& \operatorname{Ind}^{G}\left(X_{1} * X_{2} ; \mathbb{K}\right) \subset \operatorname{Ind}^{G}\left(X_{1} ; \mathbb{K}\right), \\
& \operatorname{Ind}^{G}\left(X_{1} * X_{2} ; \mathbb{K}\right) \subset \operatorname{Ind}^{G}\left(X_{2} ; \mathbb{K}\right),
\end{aligned}
$$

logo, do Corolário 3.3.3 temos que

$$
\begin{aligned}
& \operatorname{Ind}^{G}\left(X_{1} * X_{2} ; \mathbb{K}\right) \subset \operatorname{Ind}^{G_{1}}\left(X_{1} ; \mathbb{K}\right) \otimes \check{H}^{*}\left(B G_{2} ; \mathbb{K}\right), \\
& \operatorname{Ind}^{G}\left(X_{1} * X_{2} ; \mathbb{K}\right) \subset \check{H}^{*}\left(B G_{1} ; \mathbb{K}\right) \otimes \operatorname{Ind}^{G_{2}}\left(X_{2} ; \mathbb{K}\right),
\end{aligned}
$$

Portanto,

$$
\operatorname{Ind}^{G}\left(X_{1} * X_{2} ; \mathbb{K}\right) \subset\left[\operatorname{Ind}^{G_{1}}\left(X_{1} ; \mathbb{K}\right) \otimes \check{H}^{*}\left(B G_{2} ; \mathbb{K}\right)\right] \cap\left[\check{H}^{*}\left(B G_{1} ; \mathbb{K}\right) \otimes \operatorname{Ind}^{G_{2}}\left(X_{2} ; \mathbb{K}\right)\right] .
$$




\subsection{Casos Particulares de G-índices absolutos}

Apresentaremos nesta seção, técnicas para o cálculo do $G$-índice de Fadell e Husseini para alguns importantes $G$-espaços, considerando $G$ como sendo os grupos compactos de Lie: $\mathbb{Z}_{2}, S^{1}$ e $O(k)$.

\subsection{1 $\mathbb{Z}_{2}$-índice}

PRoposição 3.4.1. Todo espaço topológico que possui uma involução ${ }^{2}$ livre de pontos fixos, é um $\mathbb{Z}_{2}$-espaço com $\mathbb{Z}_{2}$-ação livre.

Demonstração: Seja $X$ um espaço topológico e $f: X \rightarrow X$ uma involução livre de pontos fixos. Então, o conjunto discreto $G=\left\{\operatorname{Id}_{X}, f\right\}$ junto com a operação composição é um grupo de Lie compacto isomorfo a $\mathbb{Z}_{2}$. Definimos

$$
\begin{aligned}
\rho: G \times X & \rightarrow X, \\
(g, x) & \mapsto g x=g(x), g \in G .
\end{aligned}
$$

Mostremos que $\rho$ é uma $G$-ação livre. Com efeito, $\operatorname{Id}_{\mathrm{X}} x=\operatorname{Id}_{\mathrm{X}}(x)=x$ e dados $g, g^{\prime} \in G$ $g\left(g^{\prime} x\right)=g\left(g^{\prime}(x)\right)=\left(g \circ g^{\prime}\right)(x)$. Além disso, se $x=g x=g(x)$ então, $g=\mathrm{Id}_{\mathrm{X}}$, desde que $f(x) \neq x, \forall x \in X$.

COROLARIO 3.4.2. A n-esfera $S^{n}$ é um $\mathbb{Z}_{2}$-espaço livre.

Demonstração: Consideremos a aplicação antipodal

$$
\begin{aligned}
A: \quad S^{n} & \rightarrow S^{n} \\
x & \mapsto-x,
\end{aligned}
$$

a qual é uma involução livre de pontos fixos, desde que

$$
A(A(x))=A(-x)=x \text { e } A(x)=-x \neq x, \forall x \in S^{n} .
$$

Logo, da Proposição 3.4.1, temos que $S^{n}$ é um $\mathbb{Z}_{2}$-espaço livre.

PROPOSIÇ̃̃o 3.4.3. O espaço classificante $B \mathbb{Z}_{2}$ é o plano real projetivo infinito-dimensional $\mathbb{R} P^{\infty}$.

Demonstração: Segue do Exemplo 2.3.18 que $E \mathbb{Z}_{2}(n)$ é o join

$$
\mathbb{Z}_{2} * \cdots * \mathbb{Z}_{2}
$$

de $(n+1)$-cópias de $\mathbb{Z}_{2}$, o qual é homeomorfo à $n$-esfera $S^{n}$. Usando o Exemplo 1.1.20, temos que

$$
E \mathbb{Z}_{2}=\lim _{\substack{\rightarrow \\ n \in \mathbb{Z}^{+}}} E \mathbb{Z}_{2}(n)=S^{\infty}
$$

Logo, por definição de espaço classificante segundo a construção de Milnor,

$$
B \mathbb{Z}_{2}=S^{\infty} / \mathbb{Z}_{2}=\mathbb{R} \mathrm{P}^{\infty} .
$$

\footnotetext{
${ }^{2}$ Uma involução é uma bijeção continua, tal que $f \circ f=I d$
} 
PROPOSIÇÃo 3.4.4. ([8, pg. 373]) $O \mathbb{Z}_{2}$-módulo de cohomologia $\breve{H}^{*}\left(B \mathbb{Z}_{2} ; \mathbb{Z}_{2}\right)$ é o anel polinomial sobre $\mathbb{Z}_{2}$, gerado pela classe característica $\omega_{1}=\omega_{1}\left(\gamma_{1}\right) \in H^{1}\left(B \mathbb{Z}_{2} \cdot \mathbb{Z}_{2}\right)$ e de grau 1 . Ou seja,

$$
\check{H}^{*}\left(B \mathbb{Z}_{2}, \mathbb{Z}_{2}\right) \simeq \mathbb{Z}_{2}\left[\omega_{1}\right] .
$$

Seja $X$ um espaço topológico paracompacto, o qual admite uma ação livre de $\mathbb{Z}_{2}$. Desde que $\mathbb{Z}_{2}\left[\omega_{1}\right]$ é um anel principal, então existe um inteiro positivo $k$ tal que

$$
\begin{aligned}
\operatorname{Ind}^{\mathbb{Z}_{2}}\left(X ; \mathbb{Z}_{2}\right) & =\operatorname{ker}\left(q_{X}^{*}: \mathbb{Z}_{2}\left[\omega_{1}\right] \rightarrow H_{\mathbb{Z}_{2}}^{*}\left(X ; \mathbb{Z}_{2}\right)\right) \\
& =\left\langle\left(\omega_{1}\right)^{k}\right\rangle .
\end{aligned}
$$

Por outro lado, o $\mathbb{Z}_{2}$-índice de Fadell e Huseini de valor numérico é dado por

$$
\begin{aligned}
\left|\operatorname{Ind}^{\mathbb{Z}_{2}}\left(X ; \mathbb{Z}_{2}\right)\right| & =\operatorname{dim}_{\mathbb{Z}_{2}}\left[\frac{\mathbb{Z}_{2}\left[\omega_{1}\right]}{\left\langle\left(\omega_{1}\right)^{k}\right\rangle}\right] \\
& =k=\min \left\{i: q_{X}^{*}\left(\left(\omega_{1}\right)^{i}\right)=0\right\} .
\end{aligned}
$$

OBSERVAÇ̃̃o 3.4.5. Observemos que o $\mathbb{Z}_{2}$-índice de Fadell e Huseini de valor numérico $\left|\operatorname{Ind}^{\mathbb{Z}_{2}}\left(X ; \mathbb{Z}_{2}\right)\right|$, coincide com o $\mathbb{Z}_{2}$-índice de Yang de $X$ (vide Yang [54] e [55] ), no caso em que $X$ é um espaço paracompacto com uma ação livre de $\mathbb{Z}_{2}$. No entanto, o $\mathbb{Z}_{2-}$ índice de Yang está definido para espaços não necessariamente paracompactos e, nesta situação, o cálculo do $\mathbb{Z}_{2}$-índice de Fadell e Huseini de valor numérico é inviabilizado, pois depende das propriedades topológicas de uma aplicação classificante. Para maiores detalhes, vide [44].

\subsubsection{O $\alpha$-índice de Fadell e Rabinowitz e o $S^{1}$-índice}

Seja $G$ um grupo de Lie compacto e $\alpha \in \check{H}^{*}(B G, \mathbb{K})$ um elemento qualquer tal que $\check{H}^{*}(B G ; \mathbb{K})=\mathbb{K}[\alpha]$. Desde que $\mathbb{K}[\alpha]$ é um anel principal, então para todo $G$-espaço paracompacto $X$ existe um inteiro positivo $k$ tal que

$$
\begin{aligned}
\operatorname{Ind}^{G}(X ; \mathbb{K}) & =\operatorname{ker}\left(q_{X}^{*}: \mathbb{K}[\alpha] \rightarrow H_{G}^{*}(X ; \mathbb{K})\right) \\
& =\left\langle\alpha^{k}\right\rangle .
\end{aligned}
$$

Por outro lado, o $G$-índice de Fadell e Huseini de valor numérico é dado por

$$
\begin{aligned}
\left|\operatorname{Ind}^{G}(X ; \mathbb{K})\right| & =\operatorname{dim}_{\mathbb{K}}\left[\frac{\mathbb{K}[\alpha]}{\left\langle\alpha^{k}\right\rangle}\right] \\
& =\min \left\{i: q_{X}^{*}\left(\alpha^{i}\right)=0\right\} .
\end{aligned}
$$

DEFINIÇ̃̃o 3.4.6. Seja $X$ um $G$-espaço paracompacto e suponhamos que $\check{H}^{*}(B G ; \mathbb{K})=$ $\mathbb{K}[\alpha]$, para algum $\alpha \in \check{H}^{*}(B G ; \mathbb{K})$. Definimos o $\alpha$-índice de Fadell e Rabinowitz de $X$ (vide [22]), o qual será denotado por

$$
\operatorname{Index}_{\alpha}^{*}(X ; \mathbb{K})
$$

como sendo o $G$-índice de Fadell e Husseini de valor numérico do $G$-espaço $X$, ou seja,

$$
\operatorname{Index}_{\alpha}^{*}(X ; \mathbb{K})=\left|\operatorname{Ind}^{G}(X ; \mathbb{K})\right| \text {. }
$$


A esfera $S^{1}=\{z \in \mathbb{C}:|z|=1\}=\mathbb{R} / \mathbb{Z}$ é um grupo de Lie compacto, com operação dada pelo produto de números complexos e com a topologia induzida de $\mathbb{C}$. O espaço $\left(\mathbb{C}^{n+1}\right)^{*}=\mathbb{C}^{n+1}-\{0\}$ é um $S^{1}$-espaço com ação dado por $\left(z,\left(z_{0}, \ldots, z_{n}\right)\right) \mapsto\left(z z_{0}, \ldots, z z_{n}\right)$. Temos que $S^{2 n+1} \subset\left(\mathbb{C}^{n+1}\right)^{*}$ é invariante por esta ação, a qual é livre e, o espaço de órbitas $S^{2 n+1} / S^{1}$ é uma variedade $2 n$-dimensional, chamada espaço projetivo complexo $\mathbb{C} P^{n}$.

PRoposição 3.4.7. Para cada $n \geq 0$, existe um inteiro positivo $k$ tal que

$$
\operatorname{Ind}^{S^{1}}\left(S^{2 n+1} ; \mathbb{Z}_{2}\right)=\left\langle\left(\omega_{2}\right)^{k}\right\rangle
$$

onde $\omega_{2} \in \check{H}^{2}\left(B S^{1} ; \mathbb{Z}_{2}\right)$ é uma classe característica de grau 2 .

Demonstração: Do exemplo 2.3.19, $E S^{1}(n)=S^{1} * \cdots * S^{1}(n+1)$-cópias é homeomorfo à $(2 n+1)$-esfera $S^{2 n+1}$. Logo, por definição de espaço classificante segundo a construção de Milnor,

$$
B S^{1}=\lim _{\substack{n \in \mathbb{Z}^{+}}} \frac{S^{2 n+1}}{S^{1}}=\lim _{\substack{n \in \mathbb{Z}^{+} \\ \rightarrow}} \mathbb{C} P^{n}=\mathbb{C} P^{\infty} .
$$

Temos que o $\mathbb{Z}_{2}$-módulo de cohomologia $\check{H}^{*}\left(B S^{1} ; \mathbb{Z}_{2}\right)$ é o anel polinomial sobre $\mathbb{Z}_{2}$, gerado por pela classe característica $\omega_{2}=\omega_{2}\left(\gamma_{2}\right) \in H^{2}\left(B S^{1} ; \mathbb{Z}_{2}\right)$ e de grau 2 (Vide [8, pg. 373]). Ou seja,

$$
\check{H}^{*}\left(B S^{1}, \mathbb{Z}_{2}\right) \simeq \mathbb{Z}_{2}\left[\omega_{2}\right] .
$$

Portanto, temos que para cada $n \geq 0$, existe um inteiro positivo $k$ tal que

$$
\operatorname{Ind}^{S^{1}}\left(S^{2 n+1} ; \mathbb{Z}_{2}\right)=\left\langle\left(\omega_{2}\right)^{k}\right\rangle
$$

\subsection{3 $O O(k)$-índice de uma Variedade de Stiefel}

Para o estudo deste $G$-índice, usaremos os seguintes artigos: Jaworowski [31] e Komiya [33]. O grupo linear geral $G L_{n}(\mathbb{R})$, onde $\mathbb{R}$ é o corpo dos números reais, é um grupo de Lie com relação à multiplicação de matrizes (composição de transformações) e com a topologia induzida de $\mathbb{R}^{n^{2}}$. Também seu subgrupo clássico $O(k)$, chamado grupo ortogonal, é um grupo de Lie. Desde que este subgrupo é fechado e limitado no espaço das matrizes, segue que $O(k)$ é compacto.

DEFINIÇÃo 3.4.8. A variedade de Stiefel denotada por $V_{k}\left(\mathbb{R}^{n}\right)$ é o espaço de $k$-frames ortonormais em $\mathbb{R}^{n}(k \leq n)$, isto é,

$$
V_{k}\left(\mathbb{R}^{n}\right)=\left\{\left(v_{1}, \cdots, v_{k}\right) \in\left(\mathbb{R}^{n}\right)^{k}:\left\langle v_{i}, v_{j}\right\rangle=\delta_{i j}, 1 \leq i j \leq k\right\} .
$$

Podemos ver que $V_{1}\left(\mathbb{R}^{n}\right)=S^{n-1}$ e $V_{k}\left(\mathbb{R}^{k}\right)=O(k)$ é o grupo ortogonal. Portanto $V_{k}\left(\mathbb{R}^{n}\right)$, é um subespaço do espaço compacto $\left(S^{n-1}\right)^{k}$. Desde que $V_{k}\left(\mathbb{R}^{n}\right)$ é um subconjunto fechado de um espaço compacto, segue que $V_{k}\left(\mathbb{R}^{n}\right)$ é compacto. Além disso, $V_{k}\left(\mathbb{R}^{n}\right)$ é uma variedade orientável de dimensão $n k-k(k+1) / 2$ e, se $k<n, V_{k}\left(\mathbb{R}^{n}\right)$ é conexa. Mostra-se que o grupo ortogonal $O(k)$ age sobre $\left(\mathbb{R}^{n}\right)^{k}$, por multiplicação de matrizes à esquerda e que esta $O(k)$-ação é livre sobre $V_{k}\left(\mathbb{R}^{n}\right)$. Denotemos o espaço de órbitas 
$V_{k}\left(\mathbb{R}^{n}\right) / O(k)$ por $G_{k}\left(\mathbb{R}^{n}\right)$, o qual chamaremos variedade Grassmanniana de dimensão $n k$.

Temos que o join $E O(k)(n)=O(k) * \cdots * O(k)$, de $n+1$ cópias do grupo ortogonal $O(k)$, é homeomorfo à variedade de Stiefel $V_{k}\left(\mathbb{R}^{n}\right)$ (vide [31]). Logo, pela definição do espaço classificante segundo a construção de Milnor,

$$
B O(k)=\lim _{\substack{n \in \mathbb{Z}^{+}}} \frac{V_{k}\left(\mathbb{R}^{n}\right)}{O(k)}=\lim _{\substack{n \in \mathbb{Z}^{+}}} G_{k}\left(\mathbb{R}^{n}\right)=G_{k}\left(\mathbb{R}^{\infty}\right) .
$$

$\mathrm{O} \mathbb{Z}_{2}$-módulo de cohomologia $\check{H}^{*}\left(B O(k) ; \mathbb{Z}_{2}\right)$ é o anel polinomial sobre $\mathbb{Z}_{2}$, gerado livremente pelas $i$-classes de Stiefel-Whitney $\omega_{1}\left(\gamma^{n}\right), \cdots, \omega_{k}\left(\gamma^{n}\right)$, onde $\omega_{i}=\omega_{i}\left(\gamma^{n}\right)$ $\in \check{H}^{i}\left(G_{k}\left(\mathbb{R}^{\infty}\right) ; \mathbb{Z}_{2}\right)$. Ou seja,

$$
H^{*}\left(B O(k), \mathbb{Z}_{2}\right) \simeq \mathbb{Z}_{2}\left[\omega_{1}, \cdots, \omega_{k}\right] .
$$

Seja $\omega=1+\omega_{1}+\omega_{2}+\cdots$ a classe de Stiefel-Whitney total e $\bar{\omega}=1+\bar{\omega}_{1}+\bar{\omega}_{2} \cdots$ sua classe dual definida pela relação $\omega \bar{\omega}=1 \mathrm{em} \mathbb{Z}_{2}\left[\omega_{1}, \cdots, \omega_{k}\right]$

PRoposição 3.4.9. Suponhamos que $\tilde{J}(k, l)$ seja o ideal em $\mathbb{Z}_{2}\left[\omega_{1}, \omega_{2}, \ldots\right]$, gerado por $\bar{\omega}_{l+1}, \bar{\omega}_{l+2} \ldots, \bar{\omega}_{l+k}$. Então,

$$
\operatorname{Ind}^{O(k)}\left(V_{k}\left(\mathbb{R}^{n}\right) ; \mathbb{Z}_{2}\right)=J(k, n-k),
$$

onde $J(k, l)$ denota a imagem de $\tilde{J}(k, l)$ pela projeção

$$
\mathbb{Z}_{2}\left[\omega_{1}, \omega_{2}, \ldots\right] \rightarrow \mathbb{Z}_{2}\left[\omega_{1}, \ldots, \omega_{k}\right] .
$$

Demonstração: Desde que $O(k)$ age livremente sobre $V_{k}\left(\mathbb{R}^{n}\right)$, segue da proposição 3.1.6 que

$$
\begin{aligned}
H_{O(k)}^{*}\left(V_{k}\left(\mathbb{R}^{n}\right) ; \mathbb{Z}_{2}\right) & \cong \check{H}^{*}\left(G_{k}\left(\mathbb{R}^{n}\right) ; \mathbb{Z}_{2}\right) \\
& =\frac{Z_{2}\left[\omega_{1}, \ldots, \omega_{k}, \bar{\omega}_{1}, \ldots, \bar{\omega}_{k}\right]}{I(k, n-k)}
\end{aligned}
$$

onde $I(k, n-k)$ é o ideal gerado pelos $n$ termos do produto

$$
\left(1+\omega_{1}+\cdots+\omega_{k}\right)\left(1+\bar{\omega}_{1}+\cdots+\bar{\omega}_{n-k}\right)
$$

de dimensão positiva. As relações correspondentes aos primeiros termos homogêneos do último produto fornecem $n-k$ equações

$$
\omega_{k}+\omega_{k-1} \bar{\omega}_{1}+\cdots+\omega_{1} \bar{\omega}_{k-1}+\bar{\omega}_{k}=0, \quad k=1, \ldots, n-k,
$$

as quais podem ser resolvidas para $\bar{\omega}_{1}, \ldots, \bar{\omega}_{n-k}$. Substituindo os resultados aos $k$ termos homogêneos restantes do produto (em dimensões $n-k+1, \ldots, n$ ) obtendo assim o ideal $J(k, n-k)$. Portanto,

$$
H_{O(k)}^{*}\left(V_{k}\left(\mathbb{R}^{n}\right) ; \mathbb{Z}_{2}\right) \cong \frac{Z_{2}\left[\omega_{1}, \ldots, \omega_{k}, \bar{\omega}_{1}, \ldots, \bar{\omega}_{k}\right]}{J(k, n-k)}
$$


Ao considerar o diagrama

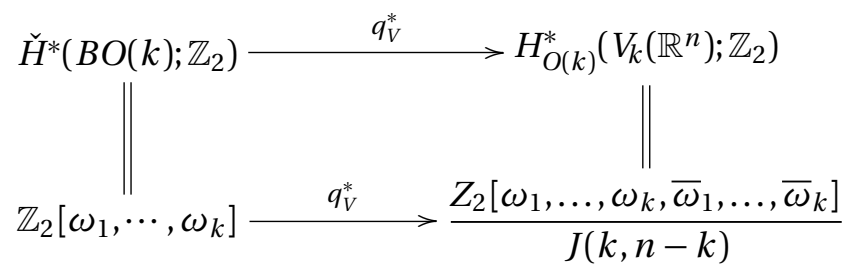

deduz-se que o $O(k)$-índice de Fadell e Husseini

$$
\operatorname{Ind}^{O(k)}\left(V_{k}\left(\mathbb{R}^{n}\right) ; \mathbb{Z}_{2}\right)=J(k, n-k) .
$$




\section{Grau de Aplicações equivariantes}

Em topologia algébrica, o grau é um invariante numérico que descreve uma aplicação contínua $f: M \rightarrow N$ entre duas variedades compactas, conexas, orientadas e de mesma dimensão. Intuitivamente, o grau representa o número de vezes que a variedade $M$ "envolve-se"na variedade $N$ sob a aplicação $f$. O grau de uma aplicação é sempre um número inteiro e foi definido pela primeira vez por Brouwer [9], que mostrou que o grau é um invariante por homotopia. Tal fato foi usado para provar o teorema do ponto fixo de Brouwer. Mais precisamente,

DEFINIÇÃo 4.0.10. Dada uma aplicação contínua $f: M \rightarrow N$ entre duas variedades orientadas compactas, conexas, sem bordo e de mesma dimensão $n$, com geradores $[M] \mathrm{e}[N]$ de $H^{n}(M ; \mathbb{Z})$ e $H^{n}(N ; \mathbb{Z})$, respectivamente, o grau de $f$ é definido como sendo o inteiro $\operatorname{deg} f$ tal que

$$
f^{*}([N])=(\operatorname{deg} f)[M] .
$$

Nosso objetivo neste capítulo é estudar este grau para aplicações $G$-equivariantes, usando o $G$-índice de Fadell e Husseini estudado no Capitulo 3.

Provavelmente, o primeiro resultado desta teoria foi o famoso teorema de LjusternikSchnirelman-Borsuk, que afirma que o grau de uma aplicação ímpar de uma esfera finito dimensional em si mesma é ímpar [6], [7] e [35]. Este resultado, foi generalizado por Eilenberg [19] para aplicações simpliciais, as quais comutam com uma ação livre simplicial de um grupo cíclico $\mathbb{Z}_{p}$, com $p$ primo, sobre esferas. Os desenvolvimentos posteriores desta teoria foram, em grande parte, devidos a P. Smith e M. Krasnoselskii.

Este capítulo está organizado como segue. Na Seção 4.1 apresentaremos o resultado de Gonçalves, Pergher, Jaworowski e Volovikov [18] sobre a existência de um homomorfismo transfer $\tau_{X}: H^{i}(X ; R) \rightarrow H^{i}(X / G ; R)$ para $G$-espaços livres, com $G$ um grupo finito. Tal homomorfismo possui a propriedade de ser sobrejetor, quando o $G$-espaço for um CW- complexo de dimensão finita. Usando tal resultado, foi possível apresentar uma demonstração alternativa do resultado provado por Hara[24] e Jaworowski [32]. Em suas demonstrações do resultado principal deste capítulo, eles utilizaram a cohomologia especial de Smith para $\mathbb{Z}_{p}$-espaços livres. A demonstração apresentada neste capítulo, utilizando o homomorfismo transfer de Gonçalves e Pergher, simplifica consideravelmente a prova deste resultado. Na Seção 4.2 apresentamos um breve resumo da teoria de 
sequências espectrais, as quais serão utilizadas na Seção 4.3 para construção de um homomorfismo transfer chamado "integração ao longo da fibra". Fazendo a composição do homorfismos transfer de Gonçalves e Pergher com o homomorfismo transfer "integração ao longo da fibra", na Seção 4.4, obtemos um novo homomorfismo transfer, fundamental na demonstração do Teorema 4.4.4, o qual será o desfecho do nosso trabalho, relacionado a Teoria de $G$-índice de Fadell e Husseini com a Teoria de Grau de aplicações G-equivariantes.

\subsection{O Homomorfismo transfer de Gonçalves e Pergher}

O objetivo desta seção é apresentar um importante resultado sobre a existência de um homomorfismo transfer $\tau_{X}: H^{i}(X ; R) \rightarrow H^{i}(X / G ; R)$ construído por Gonçalves, Pergher, Jaworowski e Volovikov em [18] a partir de $G$-espaços livres, com $G$ um grupo finito. Tal homomorfismo possui a propriedade de ser sobrejetor, quando o $G$-espaço for um CWcomplexo de dimensão finita, como mostra o seguinte

TEOREMa 4.1.1. ([18, Lemma 3, pg 64]) Sejam X um espaço de Hausdorff, conexo e localmente conexo por caminhos, e $G$ um grupo finito atuando livremente sobre $X$. Então, para todo $i \geq 0$ e $R$ um anel comutativo com identidade, existe um homomorfismo transfer $\tau_{X}: H^{i}(X ; R) \rightarrow H^{i}(X / G ; R)$ satisfazendo as seguintes propriedades:

(i) Se $X$ for um $G$-CW-complexo de dimensão $k$ (não necessariamente finito), então $\tau_{X}$ : $H^{k}(X ; R) \rightarrow H^{k}(X / G ; R)$ é sobrejetor.

(ii) Sejam $Y$ nas mesmas hipóteses do teorema, $h: X \rightarrow Y$ uma aplicação G-equivariante, $e \bar{h}: X / G \rightarrow Y / G$ a induzida da aplicação $h$ entre os espaços de órbitas. Então, $\tau_{X} \circ h^{*}=$ $\bar{h}^{*} \circ \tau_{Y}$.

Demonstração: Definimos inicialmente $\tau_{X}$ em nível de cocadeias. Seja $\mu \in C^{i}(X ; R)$ uma $i$-cadeia singular, isto é, $\mu$ é um $R$-homomorfismo do $R$-módulo de $i$-cadeias singulares $C_{i}(X ; R)$ em $R$. Consideremos o $i$-simplexo padrão $\sigma_{i}$ e fixemos um ponto base $z \in \sigma_{i}$. Seja $\phi: \sigma_{i} \rightarrow X / G$ um $i$-simplexo singular e seja

$$
\pi^{-1}(\phi(z))=\left\{a_{1}, a_{2}, \cdots, a_{r}\right\},
$$

onde $r$ é a ordem do grupo $G$ e $\pi: X \rightarrow X / G$ é a aplicação quociente. Desde que $\pi$ : $X \rightarrow X / G$ é um recobrimento a $r$-folhas, com $X$ localmente conexo por caminhos e $\sigma i$ é simplesmente conexo, existem únicos levantamentos

$$
\phi_{1}, \phi_{2}, \ldots, \phi_{r}: \sigma_{i} \rightarrow X,
$$

de $\phi$ tal que $\phi_{i}(z)=a_{i}$, para $i=1, \ldots, r$. Definimos

$$
\bar{\mu}(\phi)=\mu\left(\phi_{1}\right)+\mu\left(\phi_{2}\right)+\cdots+\mu\left(\phi_{r}\right) .
$$

Definida desta forma, temos que a aplicação $\mu \mapsto \bar{\mu}$ é $R$ linear e leva cociclos em cociclos e cobordos em cobordos e, deste modo, define um $R$-homomorfismo $\tau_{X}: H^{i}(X ; R) \rightarrow$ 
$H^{i}(X / G ; R)$ (o qual depende do ponto base $z$ de $\sigma_{i}$ ). A condição (ii) segue do fato de que $\bar{h} \circ \pi_{X}=\pi_{Y} \circ h$.

Agora, suponhamos que $X$ seja um $G$-CW-complexo de dimensão $k$. Consideremos $C^{j}(, R)=\operatorname{Hom}\left(C_{j}(, R), R\right)$, onde $C_{j}(, R)$ é o $R$-módulo livre gerado pelas $j$-células abertas. Como $X$ é um $G$-CW-complexo, temos que é induzido sobre $X / G$ uma estrutura de $C W$-Complexo. Assim, em particular se $\left\{e_{\alpha}\right\}$ são as $k$-células de $X / G$, então para cada $\alpha$ existem exatamente $r k$-células $e_{\alpha}^{1}, e_{\alpha}^{2}, \ldots, e_{\alpha}^{r}$ de $X$ mapeadas por $\pi$ sobre $e_{\alpha}$. Neste caso, o homomorfismo transfer $\tau_{X}: H^{i}(X ; R) \rightarrow H^{i}(X / G ; R)$ é o homomorfismo induzido pela aplicação de cadeias $\tau_{X}: C^{k}(X, R) \rightarrow C^{k}(X ; R)$ dada por

$$
\tau_{X}(\mu)\left(e_{\alpha}\right)=\mu\left(e_{\alpha}^{1}\right)+\mu\left(e_{\alpha}^{2}\right)+, \ldots+\mu\left(e_{\alpha}^{r}\right) .
$$

Agora, dado um $k$-cociclo $\eta \in C^{k}(X / G ; R)$, definimos uma $k$-cocadeia $\eta^{\prime} \in C^{k}(X ; R)$ por $\eta^{\prime}\left(e_{\alpha}^{j}\right)=\eta\left(e_{\alpha}\right)$, se $j=1$ e, $\eta^{\prime}\left(e_{\alpha}^{j}\right)=0$, se $j \neq 1$, para todo $\alpha$. Deste modo, temos que $\tau_{X}\left(\eta^{\prime}\right)=\eta$ e como $X$ tem dimensão $k$, segue que $C^{k+1}(X ; R)=0 \mathrm{e}$, portanto, qualquer $k$-cocadeia de $X$ é um $k$-cociclo. Então $\eta^{\prime}$ representa uma classe em $H^{k}(X ; R)$ tal que $\tau_{X}\left(\left[\eta^{\prime}\right]\right)=[\eta]$.

Se no Teorema 4.1.1, o $G$-espaço $X$ for uma variedade compacta,conexa e sem bordo e $R=\mathbb{Z}_{2}$, temos que o homomorfismo transfer $\tau_{X}$ é um isomorfismo, como mostra a seguinte

PROPOSIÇÃo 4.1.2. Suponhamos que G seja um grupo finito agindo livremente sobre uma variedade $M$ compacta, conexa e sem bordo de dimensão $m$. Então, o homomorfismo transfer $\tau_{X}: H^{m}\left(M ; \mathbb{Z}_{2}\right) \rightarrow H^{m}\left(M / G ; \mathbb{Z}_{2}\right)$ é um isomorfismo.

Demonstração: Temos que $M$ e $M / G$ admitem uma estrutura de $C W$-complexo finito, desde que ambas são variedades compactas sem bordo (vide [36, Capítulo 20, pg.149]). Além disso, sendo também conexas, segue que

$$
H^{m}\left(M ; \mathbb{Z}_{2}\right) \cong H^{m}\left(M / G ; \mathbb{Z}_{2}\right) \cong \mathbb{Z}_{2}
$$

Assim, desde que pelo Teorema 4.1.1, o homomorfismo transfer

$$
\tau_{X}: H^{m}\left(M ; \mathbb{Z}_{2}\right) \rightarrow H^{m}\left(M / G ; Z_{2}\right)
$$

é sobrejetor, concluímos que $\tau_{X}$ é um isomorfismo.

\subsection{Sequências Espectrais}

Nesta seção, daremos uma breve descrição dos resultados sobre sequências espectrais que usaremos mais adiante.

DEFINIÇão 4.2.1. Um módulo diferencial bigraduado sobre um anel $R$, é uma coleção de $R$-módulos $\left\{E^{p, q}\right\}$, para todo par de inteiros $p$ e $q$, junto com uma aplicação $R$-linear $d: E^{*, *} \rightarrow E^{*, *}$, o diferencial, de bigrau $(-r, r-1)$ ou $(r,-r+1)$, para algum inteiro $r$, satisfazendo $d \circ d=0$. 
DEFINIÇÃo 4.2.2. O módulo de homologia $H(E)$ é o módulo bigraduado

$$
H_{p, q}\left(E_{*, *}, d\right)=\frac{\operatorname{ker}\left(d: E_{p, q} \rightarrow E_{p-r, q+r-1}\right)}{\operatorname{im}\left(d: E_{p+r, q-r+1} \rightarrow E_{p, q}\right) .}
$$

DEFINIÇÃo 4.2.3. O módulo de cohomologia $H(E)$ é o módulo bigraduado

$$
H^{p, q}\left(E^{*, *}, d\right)=\frac{\operatorname{ker}\left(d: E^{p, q} \rightarrow E^{p+r, q-r+1}\right)}{\operatorname{im}\left(d: E^{p-r, q+r-1} \rightarrow E^{p, q}\right) .}
$$

DEFINIÇão 4.2.4. Uma sequência espectral do tipo homológica é uma coleção de R-módulos diferenciais bigraduados $\left\{E_{*, *}^{r}, d^{r}\right\}$, para $r=1,2, \ldots$; onde os diferenciais têm bigrau $(-r, r-1)$ e $E_{p, q}^{r+1}$ é isomorfo a $H_{p, q}\left(E_{*, *}^{r}, d^{r}\right)$.

DEFINIÇÃo 4.2.5. Uma sequência espectral do tipo cohomológica é uma coleção de Rmódulos diferenciais bigraduados $\left\{E_{r}^{*, *}, d_{r}\right\}$, para $r=1,2, \ldots$; onde os diferenciais têm bigrau $(r,-r+1)$ e $E_{r+1}^{p, q}$ é isomorfo a $H^{p, q}\left(E_{r}^{*, *}, d_{r}\right)$.

OBSERVAÇÃo 4.2.6. Embora a sequência espectral esteja indexada para $r=1,2, \ldots$, essa indexação pode começar em qualquer inteiro e, para as nossas aplicações, a sequência começa em $r=2$.

Até o final desta seção, estaremos considerando sequências espectrais do tipo cohomológicas. As definições e propriedades apresentadas a seguir, também podem ser obtidas no caso de uma sequência espectral homológica e uma exposição detalhada nesse sentido pode ser encontrada em [37, 49].

Para definir o termo limite de uma sequência espectral cohomológica, para todo $k \geq r$, denotemos por

$$
\begin{aligned}
Z_{r}^{p, q} & =\operatorname{ker}\left(d_{r}: E_{r}^{p, q} \rightarrow E_{r}^{p+r, q-r+1}\right) \\
B_{r}^{p, q} & =\operatorname{im}\left(d_{r}: E_{r}^{p-r, q+r-1} \rightarrow E_{r}^{p, q}\right) .
\end{aligned}
$$

A condição $d_{r} \circ d_{r}=0$, implica que $B_{r} \subset Z_{r} \subset E_{r}$, e segue da definição 4.2 .3 que $E_{r+1} \cong$ $Z_{r} / B_{r}$. Sejam

$$
\begin{aligned}
& Z\left(E_{r+1}\right)^{p, q}=\operatorname{ker}\left(d_{r+1}: E_{r+1}^{p, q} \rightarrow E_{r+1}^{p+r+1, q-r}\right) \\
& B\left(E_{r+1}\right)^{p, q}=\operatorname{im}\left(d_{r+1}: E_{r+1}^{p-r-1, q+r} \rightarrow E_{r+1}^{p, q}\right) .
\end{aligned}
$$

Segue de [29, Teorema 1.10, pg.173], que existem submódulos bigraduados $Z_{r+1}$ e $B_{r+1}$ de $Z_{r}$, contendo $B_{r}$, tais que $Z\left(E_{r+1}\right)^{p, q} \cong Z_{r+1}^{p, q} / B_{r}^{p, q}$ e $B\left(E_{r+1}\right)^{p, q} \cong B_{r+1}^{p, q} / B_{r}^{p, q}$, para todo $p, q$. Assim, $B_{r+1} \subset Z_{r+1}$ e temos que

$$
B_{r} \subset \quad B_{r+1} \subset Z_{r+1} \subset Z_{r} \subset E_{r} .
$$

Além disso, $E_{r+2} \cong Z\left(E_{r+1}\right) / B\left(E_{r+1}\right) \cong Z_{r+1} / B_{r+1}$. Continuando esse processo por indução, obtemos uma sequência de submódulos, para todo $n \geq r$,

$$
B_{r} \subset B_{r+1} \subset \cdots \subset B_{n} \subset \cdots \subset Z_{n} \subset \cdots \subset Z_{r+1} \subset Z_{r} \subset E_{r},
$$

com a propriedade que $E_{n+1} \cong Z_{n} / B_{n}$. 
DEFINIÇÃO 4.2.7. Definimos os módulos bigraduados

$$
Z_{\infty}=\bigcap_{n} Z_{n} \text { e } B_{\infty}=\bigcup_{n} B_{n}
$$

O módulo bigraduado $E_{\infty}=Z_{\infty} / B_{\infty}$ é chamado o limite da sequência espectral $E$.

DEFINIÇÃo 4.2.8. Uma sequência espectral cohomológica $\left\{E_{r}^{*, *}, d_{r}\right\}$ colapsa no $N$-ésimo termo se o diferencial $d_{r}=0$, para todo $r \geq N$.

OBSERVAÇÃo 4.2.9. Uma consequência imediata do fato de uma sequência espectral cohomológica $\left\{E_{r}^{*, *}, d_{r}\right\}$ colapsar no $N$-ésimo termo, é que $E_{N}^{*, *} \cong E_{N+1}^{*, *} \cong \cdots \cong E_{\infty}^{*, *}$.

DEFINIÇÃo 4.2.10. Uma filtração decrescente $F$ sobre um $R$-módulo $A$, é uma família de submódulos $\left\{F^{p}(A)\right\}$, com $p \in \mathbb{Z}$, tal que

$$
\cdots F^{p+1}(A) \subset F^{p}(A) \subset F^{p-1}(A) \subset \cdots \subset A .
$$

DEFINIÇÃo 4.2.11. Dada uma filtração decrescente $F$ sobre um $R$-módulo $A$, o módulo graduado associado $E_{0}^{*}(A)$ é dado por

$$
E_{0}^{p}(A)=F^{p}(A) / F^{p+1}(A) .
$$

OBSERVAÇão 4.2.12. Se $H^{*}$ é um $R$-módulo graduado e se $F$ é uma filtração sobre $H^{*}$, então $F^{p}\left(H^{n}\right)=F^{p}\left(H^{*}\right) \cap H^{n} \subset F^{p-1}\left(H^{*}\right) \cap H^{n}=F^{p-1}\left(H^{n}\right)$ e o módulo bigraduado associado $E_{0}^{*, *}$ é dado por

$$
E_{0}^{p, q}\left(H^{*}, F\right)=F^{p}\left(H^{p+q}\right) / F^{p+1}\left(H^{p+q}\right) .
$$

DEFINIÇÃO 4.2.13. Uma sequência espectral $\left\{E_{r}^{*, *}, d_{r}\right\}$ converge para um $R$-módulo graduado $H^{*}$, se existe uma filtração $F$ sobre $H^{*}$ tal que

$$
E_{\infty}^{p, q} \cong E_{0}^{p, q}\left(H^{*}, F\right)
$$

onde $E_{\infty}^{*, *}$ é o termo limite da sequência espectral.

DEFINIÇÃo 4.2.14. Uma sequência espectral $\left\{E_{r}^{*, *}, d_{r}\right\}$ é uma sequência espectral do primeiro quadrante, se existe $r$ tal que $E_{r}^{p, q}=0$, para $p<0$ ou $q<0$.

Agora, recordemos os seguintes teoremas de Leray-Serre para fibrações (para demonstração vide [37, Teoremas 5.2 e 6.7]).

TEOREMA 4.2.15. (A Sequência Espectral Cohomológica de Leray-Serre) Seja $R$ um anel comutativo com identidade. Dada uma fibração $F \hookrightarrow E \stackrel{p}{\rightarrow} B$, onde B é conexo por caminhos, existe uma sequência espectral do primeiro quadrante $\left\{E_{r}^{*, *}, d_{r}\right\}$, com

$$
E_{2}^{p, q} \cong H^{p}\left(B ;\left\{H^{q}(F ; R)\right\}\right)
$$

a cohomologia de B com coeficientes locais na cohomologia de $F$, a fibra de $p$, e convergindo para $H^{*}(E ; R)$. Além disso, essa sequência é natural com relação a aplicações entre fibrações que preservem fibras.

Para mais detalhes sobre sistemas de coeficientes locais vide [37]. 


\subsection{Integração ao Longo da Fibra}

Uma ferramenta importante na construção do homomorfismo transfer de grau dim $G^{1}$ é o homomorfismo integração ao longo da fibra, definido no trabalho de Gottlieb [23].

Assumiremos nesta seção, que $X$ é um $G$-espaço paracompacto com $G$-ação livre, onde $G$ é um grupo de Lie compacto e conexo. Então, do teorema 2.3.5, $(X, \pi, X / G, G, G)$ é um $G$-fibrado principal. Desde que $G$ é um grupo de Lie compacto, então $G$ pode ser visto como um subgrupo fechado do grupo ortogonal $O(n)$, para algum $n$ [8, Teo. 5.1, pag. 23] e, portanto, $G$ é orientável. Assim, segue da dualidade de Poincaré para o grupo $G$ (vide [49, Capítulo 6, Teorema 18, Pg. 297]) que

$$
H^{\operatorname{dim} G}(G ; R) \cong H_{0}(G ; R)=R \quad \text { e } \quad H^{i}(G ; R)=0 \text {, para todo } i>\operatorname{dim} G .
$$

Segue do Teorema 4.2.15, que existe uma sequência espectral de Leray-Serre associada ao $G$-fibrado principal $\pi: X \rightarrow X / G$ que se comporta da seguinte forma:

$$
E_{r}^{q-\operatorname{dim} G-r, \operatorname{dim} G+r-1} \stackrel{d_{r}}{\longrightarrow} E_{r}^{q-\operatorname{dim} G, \operatorname{dim} G}
$$

é zero para $r>1 \mathrm{e}$

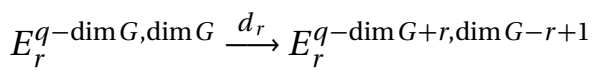

é zero para $r>\operatorname{dim} G+1$. Portanto

$$
E_{2}^{q-\operatorname{dim} G, \operatorname{dim} G} \supset E_{3}^{q-\operatorname{dim} G, \operatorname{dim} G} \supset \cdots \supset E_{\operatorname{dim} G+2}^{q-\operatorname{dim} G \operatorname{dim} G}=E_{\infty}^{q-\operatorname{dim} G, \operatorname{dim} G}
$$

Também $E_{2}^{q-i, i}=H^{q-i}\left(B ; H^{i}(G ; R)\right)=0$, para $i>\operatorname{dim} G$ então $E_{\infty}^{q-i, i}$ também é zero, para $i>\operatorname{dim} G$. Portanto, a filtração padrão

$$
H^{q}(X ; R)=F^{-1, q+1} \supset \cdots \supset F^{i, q-i} \supset \cdots
$$

reduz-se

$$
H^{q}(X ; R) \supset F^{q-\operatorname{dim} G, \operatorname{dim} G} \supset 0
$$

assim

$$
H^{q}(X ; R) / F^{q-\operatorname{dim} G, \operatorname{dim} G} \cong E_{\infty}^{q-\operatorname{dim} G, \operatorname{dim} G}
$$

DEFINIÇão 4.3.1. Seja $X$ um $G$-espaço paracompacto, com $G$ conexo. O homomorfismo integração ao longo da fibra

$$
\varpi: H^{q}(X ; R) \rightarrow H^{q-\operatorname{dim} G}(X / G ; R)
$$

é definido como sendo a seguinte composição:

$$
\varpi: H^{q}(X ; R) \rightarrow E_{\infty}^{q-\operatorname{dim} G, \operatorname{dim} G} \nrightarrow E_{2}^{q-\operatorname{dim} G, \operatorname{dim} G} \cong H^{q-\operatorname{dim} G}(X / G ; R)
$$

${ }^{1}$ dim denota dimensão topológica. 
PRoposição 4.3.2. Sejam X e $Y$, G-espaços paracompactos, com $G$ conexo. Se $f: X \rightarrow Y$ for uma aplicação G-equivariante, então o seguinte diagrama é comutativo

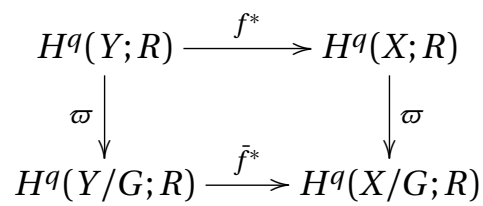

Demonstração: Segue do fato de que a sequência espectral dada pelo Teorema 4.2.15 é natural com relação a aplicações entre fibrações que preservam fibras.

No caso em que $X=M$ é uma variedade compacta, conexa e sem bordo de dimensão $m$ e o anel $R$ é o corpo $\mathbb{Z}_{2}$, temos a seguinte importante

PROPOSIÇão 4.3.3. Suponhamos que G seja um grupo de Lie compacto e conexo de dimensão finita $k$, que age livremente sobre uma variedade $M$ compacta, conexa e sem bordo de dimensão $m$. Então, a integração ao longo da fibra $\varpi: H^{m}\left(M ; \mathbb{Z}_{2}\right) \rightarrow H^{m-\operatorname{dim} G}\left(M / G ; \mathbb{Z}_{2}\right)$ é um isomorfismo.

Demonstração: Segue do Teorema 4.2.15 que existe uma seqüência espectral do primeiro quadrante $\left\{E_{r}^{*, *}, d_{r}\right\}$, com

$$
E_{2}^{i, j} \cong H^{i}\left(M / G ; H^{j}\left(G ; \mathbb{Z}_{2}\right)\right)
$$

convergindo para $H^{i+j}\left(M ; \mathbb{Z}_{2}\right)$. Temos que os módulos $E_{2}^{i, j}$ são nulos para $i+j=m$, exceto $E_{2}^{m-k, k}$. De fato, se $j<k$ então $m-j>m-k=\operatorname{dim} M / G$. Assim,

$$
E_{2}^{m-j, j} \cong H^{m-j}\left(M / G ; H^{j}\left(G ; \mathbb{Z}_{2}\right)\right) \cong 0 .
$$

Se $j>k=\operatorname{dim} G$, temos que $E_{2}^{m-j, j} \cong H^{m-j}\left(M / G ; H^{j}\left(G ; \mathbb{Z}_{2}\right)\right) \cong 0$. Além disso, os diferenciais com domínio $E_{2}^{m-k, k}$ e imagem $E_{2}^{m-k, k}$ são nulos. Assim, usando o fato que

$$
H^{m}\left(M ; \mathbb{Z}_{2}\right) \cong H^{m-k}\left(M / G ; \mathbb{Z}_{2}\right) \cong H^{k}\left(G ; \mathbb{Z}_{2}\right) \cong \mathbb{Z}_{2},
$$

temos que o homomorfismo integração ao longo da fibra:

$\varpi: \mathbb{Z}_{2} \cong H^{m}\left(M ; \mathbb{Z}_{2}\right) \cong E_{\infty}^{m-k, k} \mapsto E_{2}^{m-k, k} \cong H^{m-k}\left(M ; H^{k}\left(G ; \mathbb{Z}_{2}\right)\right) \cong H^{m-k}\left(M ; \mathbb{Z}_{2}\right) \cong \mathbb{Z}_{2}$,

é um isomorfismo.

\subsection{Relação entre o Grau e o G-índice de Fadell e Husseini}

Nesta seção, assumiremos o fato de que, se $X$ é uma variedade, então o $R$-módulo de cohomologia de Alexander é isomorfo ao $R$-módulo de cohomologia singular [49, Corolário 6.9.7, pag. 341] e, portanto, pela Proposição 1.3.24

$$
\check{H}^{q}(X ; R) \cong H^{q}(X ; R)
$$


onde $H^{q}$ denota o $R$-módulo de cohomologia singular. Dessa forma, os resultados provados anteriormente usando a cohomologia de Čech, serão considerados naturalmente na cohomologia singular.

As principais referências nesta seção, são os artigos de Hara [24] e Jaworowski [32]. Usando as definições das seções anteriores vamos construir um novo homomorfismo que será de suma importância nas demonstrações dos teoremas principais deste capítulo.

Sejam $X$ um $G$-espaço paracompacto, com $G$-ação livre e $G_{0}$ a componente conexa de $G$ contendo a identidade $e$. Sabemos do Corolário 2.1.8 que o grupo quociente $G / G_{0}$ é finito e, além disso, age livremente sobre $X / G_{0}$ da seguinte forma

$$
\left(g G_{0}, G_{0} x\right) \mapsto G_{0}(g x)
$$

Desde que $G / G_{0}$ é um grupo finito, então $\operatorname{dim}\left(G / G_{0}\right)=0$ implicando que $\operatorname{dim} G=\operatorname{dim} G_{0}$ e $\operatorname{dim}\left(X / G_{0}\right)=\operatorname{dim}(X / G)$. Por outro lado, a $G$-ação livre de $X$, induz uma $G_{0}$-ação livre sobre $X$ mediante a restrição. Então, temos o $G_{0}$-fibrado principal $\left(X, \pi, X / G_{0}, G_{0}, G_{0}\right)$ $\operatorname{com} X / G_{0}$ paracompacto (Teorema 2.3.5). Logo, da Definição 4.3.1 para o $G_{0}$-espaço paracompacto temos

$$
\varpi: H^{i}(X ; R) \rightarrow H^{i-\operatorname{dim} G}\left(X / G_{0} ; R\right)
$$

Pela Teorema 4.1.1, para o $G / G_{0}$-espaço paracompacto $X / G_{0}$ temos

$$
\tau: H^{i-\operatorname{dim} G}\left(X / G_{0} ; R\right) \rightarrow H^{i-\operatorname{dim} G}\left(\frac{X / G_{0}}{G / G_{o}}\right) \cong H^{i-\operatorname{dim} G}(X / G ; R)
$$

Este último isomorfismo deve-se ao fato que $\frac{X / G_{0}}{G / G_{o}}$ é homeomorfo a $X / G$.

DEFINIÇÃo 4.4.1. Seja $X$ um $G$-espaço paracompacto com $G$-ação livre. Definimos o homomorfismo transfer, denotado por $p$ !, dado pela composição

$$
H^{q}(X ; R) \stackrel{\varpi}{\longrightarrow} H^{q-\operatorname{dim} G}\left(X / G_{0} ; R\right) \stackrel{\tau}{\longrightarrow} H^{q-\operatorname{dim} G}(X / G ; R)
$$

PROPOSIÇÃo 4.4.2. Seja $f: X \rightarrow Y$ uma aplicação equivariante entre $G$-espaços paracompactos, com $G$-ações livres. Então, temos o seguinte diagrama comutativo

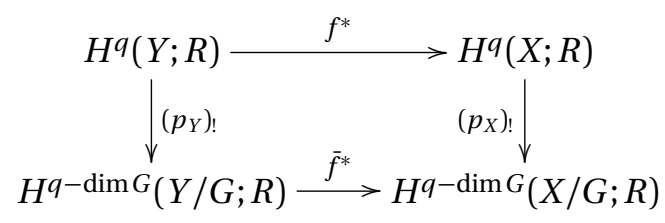

onde $\bar{f}: X / G \rightarrow Y / G$ é a aplicação induzida por $f$ entre os espaços de órbitas.

Demonstração: Para isso consideremos o seguinte diagrama

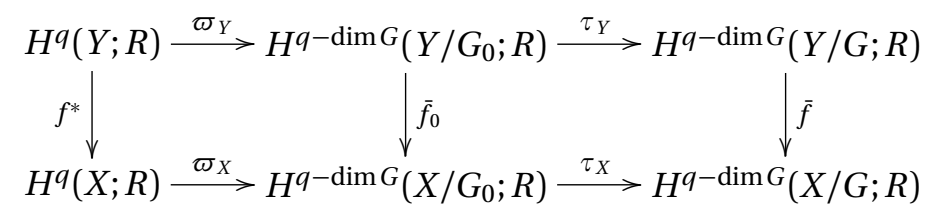


onde $\bar{f}_{0}$ é a aplicação induzida nos espaços órbitas em relação ao grupo $G_{0}$. Do Teorema 4.1.1 e da Proposição 4.3.2 temos que os quadrados são comutativos, o que implica que

$$
\bar{f}^{*} \circ\left(p_{Y}\right)_{!}=\bar{f}^{*} \circ \tau_{Y} \circ \varpi_{Y}=\tau_{X} \circ \bar{f}_{0}^{*} \circ \varpi_{Y}=\tau_{X} \circ \varpi_{X} \circ f^{*}=\left(p_{X}\right) ! \circ f^{*}
$$

A seguinte proposição é essencial na demonstração do teorema principal desta seção.

PROPOSIÇão 4.4.3. Suponhamos que G seja um grupo de Lie compacto de dimensão finita que age livremente sobre uma variedade $M$ compacta, conexa e sem bordo de dimensão $m$. Então, o homomorfismo transfer

$$
p_{!}: H^{m}\left(M ; \mathbb{Z}_{2}\right) \rightarrow H^{m-\operatorname{dim} G}\left(M / G ; \mathbb{Z}_{2}\right)
$$

é um isomorfismo.

Demonstração: Temos a decomposição

$$
H^{q}\left(M ; \mathbb{Z}_{2}\right) \stackrel{\varpi}{\longrightarrow} H^{q-\operatorname{dim} G}\left(M / G_{0} ; \mathbb{Z}_{2}\right) \stackrel{\tau}{\longrightarrow} H^{q-\operatorname{dim} G}\left(\frac{M / G_{0}}{G / G_{0}} ; \mathbb{Z}_{2}\right) \cong H^{q-\operatorname{dim} G}\left(M / G ; \mathbb{Z}_{2}\right) .
$$

Desde que $G_{0}$ é um grupo de compacto Lie, conexo e de dimensão finita que age livremente sobre $M$, então pela Proposição 4.3.3 temos que $\varpi$ é um isomorfismo. Por outro lado, desde que $G / G_{0}$ é um grupo finito que age livremente sobre a variedade compacta, conexa e sem bordo $M / G_{0}$ de dimensão $m-\operatorname{dim} G_{0}$, então pela Proposição 4.1.2, $\tau$ é um isomorfismo, e portanto, $p_{!}=\tau \circ \varpi$ é um isomorfismo.

Enunciaremos agora, o teorema principal deste capítulo, o qual será o desfecho do nosso trabalho, relacionando a Teoria de $G$-índice de Fadell e Husseini com a Teoria de Grau de aplicações G-equivariantes.

TEOREMA 4.4.4. Sejam $G$ um grupo compacto de Lie e $M, N G$-variedades compactas, conexas e sem bordo de dimensão finita $n$, que admitem uma $G$-ação livre. Então,

(1) $\operatorname{Se} \operatorname{Ind}_{n-\operatorname{dim} G}^{G}\left(N, \mathbb{Z}_{2}\right)=\operatorname{Ind}_{n-\operatorname{dim} G}^{G}\left(M ; \mathbb{Z}_{2}\right) \neq H^{n-\operatorname{dim} G}\left(B G ; \mathbb{Z}_{2}\right)$, então para toda aplicação $G$-equivariante $f: M \rightarrow N$, temos que

$$
f^{*}: H^{n}\left(N ; \mathbb{Z}_{2}\right) \rightarrow H^{n}\left(M ; \mathbb{Z}_{2}\right)
$$

é um isomorfismo. Além disso, se $M$ e $N$ são orientáveis, então para toda aplicação Gequivariante $f: M \rightarrow N$, o grau de $f$ é ímpar.

(2) $\operatorname{Se} \operatorname{Ind}_{n-\operatorname{dim} G}^{G}\left(N, \mathbb{Z}_{2}\right) \neq \operatorname{Ind}_{n-\operatorname{dim} G}^{G}\left(M ; \mathbb{Z}_{2}\right)=H^{n-\operatorname{dim} G}\left(B G ; \mathbb{Z}_{2}\right)$, então para toda aplicação $G$-equivariante $f: M \rightarrow N$, temos que

$$
f^{*}: H^{n}\left(N ; \mathbb{Z}_{2}\right) \rightarrow H^{n}\left(M ; \mathbb{Z}_{2}\right)
$$

é o homorfismo trivial. Além disso, se $M$ e $N$ são orientáveis, então para toda aplicação $G$-equivariante $f: M \rightarrow N$, o grau de fé par. 
Demonstração: (1) Sejam $q_{M}: M / G \rightarrow B G$ e $q_{N}: N / G \rightarrow B G$ as aplicações classificantes para $M$ e $N$, respectivamente. Por hipótese, usando o Teorema 3.2.9 temos que

$$
\operatorname{ker} q_{M}^{*}=\operatorname{ker} q_{N}^{*} \neq H^{n-\operatorname{dim} G}\left(B G ; \mathbb{Z}_{2}\right),
$$

ou seja, $q_{M}^{*}$ e $q_{N}^{*}$ são não nulos. Agora, considerando o diagrama comutativo

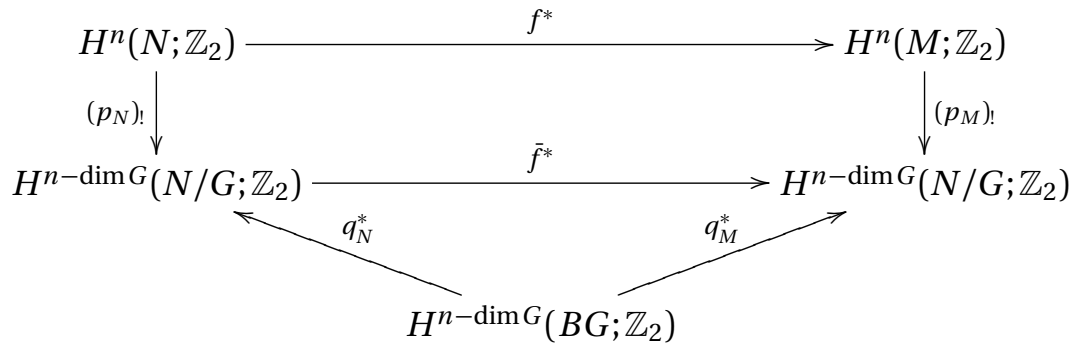

Como $\bar{f}^{*} q_{N}^{*}=q_{M}^{*}$ (Teorema 2.3.13) e $q_{M}^{*} \neq 0$, então $\bar{f}^{*} \neq 0$. Pela Proposição 4.4.3, temos que $\left(p_{M}\right)$ ! e $\left(p_{N}\right)$ ! são isomorfismos e, assim, usando o quadrado comutativo temos que $f^{*} \neq 0$. Com efeito, como $\bar{f}^{*} \neq 0$, existe

$$
0 \neq \bar{u} \in H^{n-\operatorname{dim} G}\left(N / G ; \mathbb{Z}_{2}\right)
$$

tal que $\bar{f}^{*} \bar{u} \neq 0$. Logo, pela sobrejetividade de $\left(p_{N}\right)$ ! existe

$$
0 \neq u \in H^{n}\left(N ; \mathbb{Z}_{2}\right)
$$

tal que $\left(p_{N}\right) !(u)=\bar{u}$. Assim, segue que

$$
0 \neq \bar{f}^{*}\left(p_{N}\right) !(u)=\left(p_{M}\right) ! f^{*}(u)
$$

e, da injetividade de $\left(p_{M}\right)$ !, concluímos que $f^{*}(u) \neq 0$. Agora, como

$$
H^{n}\left(N ; \mathbb{Z}_{2}\right)=H^{n}\left(M ; \mathbb{Z}_{2}\right)=\mathbb{Z}_{2},
$$

pois $M$ e $N$ são compactas, conexas e sem bordo de dimensão $n$ e

$$
f^{*}: H^{n}\left(N ; \mathbb{Z}_{2}\right) \rightarrow H^{n}\left(M ; \mathbb{Z}_{2}\right)
$$

é não trivial, concluímos que $f^{*}$ é um isomorfismo. Suponhamos agora que $M$ e $N$ sejam orientáveis e, que o grau de $f$ seja igual a $2 k$, com $k$ inteiro. Levando a módulo 2 , temos que $f^{*}: H^{n}\left(N, \mathbb{Z}_{2}\right) \rightarrow H^{n}\left(M, \mathbb{Z}_{2}\right)$ é nula, contradizendo o resultado anterior. Portanto, o grau de $f$ é ímpar.

(2) Por hipótese, usando o Teorema 3.2.9 temos que

$$
\operatorname{ker} q_{N}^{*} \neq \operatorname{ker} q_{M}^{*}=H^{n-\operatorname{dim} G}\left(B G ; \mathbb{Z}_{2}\right),
$$

ou seja, $q_{M}^{*}=0$ e $q_{N}^{*}$ é não nulo. Com isto, podemos provar que $\bar{f}^{*}$ é não injetiva. Com efeito, como $q_{N}^{*}$ é não nulo, seja $x \in H^{n-\operatorname{dim} G}\left(B G ; \mathbb{Z}_{2}\right)$ tal que $q_{N}^{*}(x) \neq 0$. Logo, usando o diagrama 4.5 temos que

$$
\operatorname{ker}\left(\bar{f}^{*} q_{N}^{*}\right)=\operatorname{ker} q_{M}^{*}=H^{n-\operatorname{dim} G}\left(B G ; \mathbb{Z}_{2}\right)
$$


ou seja, $\bar{f}^{*} q_{N}^{*}$ é nula e, portanto, $\bar{f}^{*} q_{N}^{*}(x)=0$. Além disso,

$$
H^{n-\operatorname{dim} G}\left(M / G ; \mathbb{Z}_{2}\right)=H^{n-\operatorname{dim} G}\left(N / G ; \mathbb{Z}_{2}\right)=\mathbb{Z}_{2}
$$

pois $M / G$ e $N / G$ são variedades compactas, conexas e sem bordo de dimensão $n-\operatorname{dim} G$. Como

$$
\bar{f}^{*}: H^{n-\operatorname{dim} G}\left(N / G ; \mathbb{Z}_{2}\right) \rightarrow H^{n-\operatorname{dim} G}\left(M / G ; \mathbb{Z}_{2}\right)
$$

é não injetiva, concluímos que $\bar{f}$ é trivial. Agora, usando os isomorfismos $\left(p_{M}\right)$ ! e $\left(p_{N}\right)$ ! temos que

$$
\left(p_{M}\right) !\left(f^{*}(u)\right)=\bar{f}^{*}\left(\left(p_{N}\right) !(u)\right)=0
$$

o que implica $f^{*}(u)=0$, para todo $u \in H^{n}\left(N, \mathbb{Z}_{2}\right)$. Portanto, $f^{*}: H^{n}\left(N ; \mathbb{Z}_{2}\right) \rightarrow H^{n}\left(M ; \mathbb{Z}_{2}\right)$ é o homomorfismo trivial. Suponhamos agora que $M$ e $N$ sejam orientáveis e, que o grau de $f$ seja igual a $2 k+1, \operatorname{com} k$ inteiro. Levando a módulo 2 , temos que

$$
f^{*}: H^{n}\left(N, \mathbb{Z}_{2}\right) \rightarrow H^{n}\left(M, \mathbb{Z}_{2}\right)
$$

é não trivial, o que é uma contradição. Portanto, o grau de $f$ é par. 


\section{Fatos Algébricos e Topológicos}

Este apêndice introduz as noções básicas e notações que serão usadas em todo este trabalho. Introduziremos a linguagem de categorias e funtores, espaços paracompactos e join de espaços topológicos. Uma discussão mais completa destes resultados, incluindo provas e aplicações, pode ser encontrada nas seguintes referências [14], [17], [25], [42],[43], [53].

\section{A.1 Pares de espaços e aplicações de pares}

DEFINIÇÃo A.1.1. Um par de espaços $(X, A)$ consiste de um espaço topológico $X$ e um subespaço $A \subset X$.

DEFInIÇão A.1.2. Dados pares de espaços $(X, A)$ e $(Y, B)$, uma aplicação de pares $f:(X, A) \rightarrow(Y, B)$ é uma função contínua $f: X \rightarrow Y$ tal que $f(A) \subset B$.

DEFINIÇÃo A.1.3. Duas aplicações de pares $f_{0}, f_{1}:(X, A) \rightarrow(Y, B)$ são homotópicas como aplicações de pares, se existe uma aplicação de pares

$$
F:(X \times I, A \times I) \rightarrow(Y, B)
$$

tal que $F(x, 0)=f_{0}(x)$ e $F(x, 1)=f_{1}(x)$, para todo $x \in X$.

\section{A.2 Categorias e Funtores}

DEFINIÇão A.2.1. Uma Categoria C consiste de:

(1) Uma classe de objetos $X$.

(2) Para cada par ordenado $(X, Y)$ de objetos de $\mathscr{C}$, um conjunto Hom $(X, Y)$ de morfismos $f$. Se $f \in \operatorname{Hom}(\mathrm{X}, \mathrm{Y})$, escrevemos $f: X \rightarrow Y$.

(3) Uma função, chamada composição de morfismos,

$$
: \operatorname{Hom}(\mathrm{X}, \mathrm{Y}) \times \operatorname{Hom}(\mathrm{Y}, \mathrm{Z}) \rightarrow \operatorname{Hom}(\mathrm{X}, \mathrm{Z})
$$


a qual está definida para toda tripla $(X, Y, Z)$ de objetos de $\mathbf{C}$. Dados quaisquer dois morfismos $f: X \rightarrow Y$ e $g: Y \rightarrow Z$, a imagem do par $(f, g)$ pela operação de composição será denotada por $g \circ f: X \rightarrow Z$ e os seguintes axiomas devem ser satisfeitos:

Axioma 1.(Associatividade) Dados morfismos $f: W \rightarrow X, g: X \rightarrow Y$ e $h: Y \rightarrow Z$, então: $h \circ(g \circ f)=(h \circ g) \circ f$.

Axioma 2.(Existência de identidades) Para cada objeto $X$, existe um elemento $I d_{X}: X \rightarrow$ $X$ tal que

$$
I d_{X} \circ f=f \text { e } g \circ I d_{X}=g
$$

para todo morfismo $f \in \operatorname{Hom}(\mathrm{W}, \mathrm{X})$ e para todo morfismo $g \in \operatorname{Hom}(\mathrm{X}, \mathrm{Y})$, onde $W$ e $Y$ são arbitrários.

OBSERVAÇão A.2.2. O morfismo identidade $I d_{X} \in \operatorname{Hom}(\mathrm{X}, \mathrm{X})$ é único (vide [43, pg. 155]).

Nos exemplos a seguir, listamos as categorias básicas que estaremos considerando neste trabalho, a partir das quais serão definidas novas categorias.

EXeMPlo A.2.3. A categoria dos espaços topológicos (às vezes chamados apenas de espaços) e das funções contínuas será denotada por Top.

EXEMPlo A.2.4. Pares de espaços e suas aplicações de pares constituem uma categoria, a qual será denotada por Top $^{2}$. Note que podemos associar a cada espaço topológico $X$, o par $(X, \emptyset)$ e a cada função contínua $f: X \rightarrow Y$ a correspondente aplicação de pares $f:(X, \emptyset) \rightarrow(Y, \emptyset)$.

DEFInição A.2.5. Dados morfismos $f \in \operatorname{Hom}(\mathrm{X}, \mathrm{Y})$ e $g, g^{\prime} \in \operatorname{Hom}(\mathrm{Y}, \mathrm{X})$, se $g \circ f=I d_{X}, g$ é chamado um morfismo inverso à esquerda para $f$ e se $f \circ g^{\prime}=I d_{Y}, g^{\prime}$ é chamado um morfismo inverso à direita para $f$.

OBSERVAÇÃO A.2.6. Observemos que se $f$ possui morfismo inverso à esquerda $g$ e inverso à direita $g^{\prime}$, então $g=g^{\prime}$ (vide [43, pg.156]). Nesse caso, $g$ é chamado morfismo inverso para $f$. Além disso, mostra-se que $g$ é único.

DEFINIÇÃo A.2.7. Se $f$ possui morfismo inverso, então $f$ é chamado uma equivalência na categoria em questão.

OBSERVAÇ̃̃o A.2.8. Uma equivalência na categoria Top é chamada um homeomorfismo.

DEFINIÇÃo A.2.9. Um funtor covariante $\mathfrak{F}$ de uma categoria $\mathbf{C}$ em uma categoria $\mathbf{D}$ é uma função, a qual será denotada por $\mathfrak{F}: \mathbf{C} \rightarrow \mathbf{D}$, que associa a cada objeto $X$ de $\mathbf{C}$, um objeto $\mathfrak{F}(X)$ em $\mathbf{D}$ e, a cada morfismo $f: X \rightarrow Y$ de $\mathbf{C}$, um morfismo $\mathfrak{F}(f): \mathfrak{F}(X) \rightarrow \mathfrak{F}(Y)$ de $\mathbf{D}$, satisfazendo as seguintes condições:

(i) $\mathfrak{F}\left(I d_{X}\right)=I d_{\mathfrak{F}(X)}$, para todo objeto $X$ de $\mathbf{C}$.

(ii) $\mathfrak{F}(g \circ f)=\mathfrak{F}(g) \circ \mathfrak{F}(f)$, para todo morfismo $X \stackrel{f}{\rightarrow} Y \stackrel{g}{\rightarrow} Z$ em C.

Ou seja, um funtor covariante deve preservar composições e identidades. 
OBSERVAÇÃo A.2.10. Seja $\mathfrak{F}: \mathbf{C} \rightarrow \mathbf{D}$ um funtor covariante. Observemos que se $f: X \rightarrow Y$ for uma equivalência na categoria $\mathbf{C}$, então existe um único morfismo $g$ : $Y \rightarrow X$ tal que $g \circ f=I d_{X}$ e $f \circ g=I d_{Y}$. Assim, segue da Definição A.2.9 que $\mathfrak{F}(g) \circ \mathfrak{F}(f)=$ $\mathfrak{F}(g \circ f)=\mathfrak{F}\left(I d_{X}\right)=I d_{\mathfrak{F}(X)}$ e $\mathfrak{F}(f) \circ \mathfrak{F}(g)=\mathfrak{F}(f \circ g)=\mathfrak{F}\left(I d_{Y}\right)=I d_{\mathfrak{F}(Y)}$ e, portanto, $\mathfrak{F}(f)$ será uma equivalência na categoria $\mathbf{D}$.

DEFINIÇão A.2.11. Um funtor contravariante $\mathfrak{F}$ de uma categoria $\mathbf{C}$ em uma categoria $\mathbf{D}$ é uma função, a qual será denotada por $\mathfrak{F}: \mathbf{C} \rightarrow \mathbf{D}$, que associa a cada objeto $X$ de $\mathbf{C}$, um objeto $\mathfrak{F}(X)$ em $\mathbf{D}$ e, a cada morfismo $f: X \rightarrow Y$ de $\mathbf{C}$, um morfismo $\mathfrak{F}(f): \mathfrak{F}(Y) \rightarrow \mathfrak{F}(X)$ de D, satisfazendo as seguintes condições:

(i) $\mathfrak{F}\left(I d_{X}\right)=I d_{\mathfrak{F}(X)}$, para todo objeto $X$ de $\mathbf{C}$.

(ii) $\mathfrak{F}(g \circ f)=\mathfrak{F}(f) \circ \mathfrak{F}(g)$, para todo morfismo $X \stackrel{f}{\rightarrow} Y \stackrel{g}{\rightarrow} Z$ em C.

OBSERVAÇÃo A.2.12. Exemplos importantes de funtores contravariantes que estaremos considerando neste trabalho serão apresentados no Apêndice B (Cohomologia Simplicial e Cohomologia de Čech) e no Capítulo 2 (Cohomologia G-equivariante de Borel).

\section{A.3 A Categoria dos Pares Paracompactos: Top ${ }_{p c}^{2}$}

Os resultados apresentados nesta seção podem ser encontrados em [17].

DEFINIÇÃo A.3.1. Seja $\mathscr{U}=\left\{U_{\alpha}: \alpha \in \mathfrak{A}\right\}$ uma cobertura de um espaço topológico $X$. Um refinamento de $\mathscr{U}$ é uma cobertura $\mathscr{V}=\left\{V_{\beta}: \beta \in \mathfrak{B}\right\}$ de $X$ tal que para cada $\beta \in \mathfrak{B}$, existe $\alpha \in \mathfrak{A}$ com $V_{\beta} \subset U_{\alpha}$. Se $\mathscr{V}$ for um refinamento de uma cobertura $\mathscr{U}$ de $X$, dizemos que $\mathscr{V}$ refina $\mathscr{U}$.

OBSERVAÇÃo A.3.2. Se os elementos de $\mathscr{V}$ são subconjuntos abertos de $X$, dizemos que $\mathscr{V}$ é um refinamento aberto de $\mathscr{U}$. Se os elementos de $\mathscr{V}$ são subconjuntos fechados de $X$, dizemos que $\mathscr{V}$ é um refinamento fechado de $\mathscr{U}$.

DEFINIÇão A.3.3. Uma cobertura $\mathscr{U}=\left\{U_{\alpha}: \alpha \in \mathfrak{A}\right\}$ de um espaço topológico $X$ é chamada localmente finita se para cada $x \in X$, existe um subconjunto aberto $V(x) \subset X \operatorname{com} x \in$ $V(x)$ tal que $U_{\alpha} \cap V(x) \neq \emptyset$, apenas para um número finito de índices $\alpha \in \mathfrak{A}$, ou seja, o conjunto

$$
\left\{\alpha \in \mathfrak{A}: U_{\alpha} \cap V(x) \neq \emptyset\right\}
$$

é finito.

OBSERVAÇÃo A.3.4. Se $\mathscr{U}=\left\{U_{\alpha}: \alpha \in \mathfrak{A}\right\}$ for uma cobertura localmente finita de um espaço topológico $X$, então

$$
\overline{\bigcup_{\alpha \in \mathfrak{A}} U_{\alpha}}=\bigcup_{\alpha \in \mathfrak{A}} \overline{U_{\alpha}}
$$

(Vide [42, Lema 39.1]).

DEFINIÇÃo A.3.5. Um espaço de Hausdorff $X$ é paracompacto se toda cobertura aberta de $X$ possui um refinamento aberto localmente finito. 
OBSERVAÇÃo A.3.6. Como parte da definição do termo paracompacto, seguindo a convenção de Bourbaki, estamos incluindo a hipótese de que o espaço deve satisfazer a condição de Hausdorff.

PROPOSIÇÃo A.3.7. Todo espaço compacto Hausdorffé paracompacto.

Demonstração: Seja $X$ um espaço compacto Hausdorff. Dada uma cobertura aberta $\mathscr{U}=\left\{U_{\alpha} ; \alpha \in \mathfrak{A}\right\}$ de $X$, desde que $X$ é compacto, existe uma subcobertura finita $\mathscr{V}=$ $\left\{U_{\alpha_{1}}, U_{\alpha_{2}}, \cdots, U_{\alpha_{n}}\right\}$ de $\mathscr{U}$, a qual refina $\mathscr{U}$. Além disso, dado $x \in X$, para todo aberto $V(x)$ de $X$ contendo $x$, temos que

$$
V(x) \subset \bigcup_{i=1}^{n} U_{\alpha_{i}}=X,
$$

ou seja, $V(x)$ intercepta apenas uma quantidade finita de abertos de $\mathscr{V}$ e, portanto, $X$ é paracompacto.

OBSERVAÇ̃̃o A.3.8. Se $X$ for um espaço regular então $X$ é paracompacto se, e somente se toda cobertura aberta de $X$ possui um refinamento fechado localmente finito. Esse resultado foi provado por Ernest Michael em [38, Pg.831, Lema 1] (Vide também [17, Pg.163, Teorema 2.3]).

PROPOSIÇão A.3.9. Todo subespaço fechado de um espaço paracompacto é paracompacto.

Demonstração: Sejam $Y$ um subespaço fechado de um espaço paracompacto $X$ e $\mathscr{V}=$ $\left\{V_{\alpha}, \alpha \in \mathfrak{A}\right\}$ uma cobertura aberta de $Y$ por subconjuntos abertos em $Y$. Para cada $V_{\alpha} \in \mathscr{V}$, escolhamos um aberto $U_{\alpha}$ de $X$ tal que $U_{\alpha} \cap Y=V_{\alpha}$. Então, a coleção

$$
\mathscr{U}=\left\{U_{\alpha}: U_{\alpha} \cap Y=V_{\alpha} \in \mathscr{V}\right\} \cup\{X-Y\}
$$

é uma cobertura aberta de $X$ e, desde que $X$ é paracompacto, existe um refinamento aberto localmente finito $\mathscr{W}=\left\{W_{\beta}, \beta \in \mathfrak{B}\right\}$ de $\mathscr{U}$. Mostremos que a coleção

$$
\mathscr{W}_{Y}=\left\{W_{\beta} \cap Y: W_{\beta} \in \mathscr{W}\right\}
$$

é um refinamento aberto localmente finito de $\mathscr{V}$. Com efeito, dado $W_{\beta} \in \mathscr{W}$, desde que $\mathscr{W}$ refina $\mathscr{U}$, existe um aberto $U_{\alpha} \in \mathscr{U}$ tal que $W_{\beta} \subset U_{\alpha}$, logo $W_{\beta} \cap Y \subset U_{\alpha} \cap Y=V_{\alpha}$, o que mostra que $\mathscr{W}_{Y}$ refina $\mathscr{V}$. Além disso, dado $y \in Y \subset X$, desde que $\mathscr{W}$ é localmente finito, existe um aberto $V(y)$ em $X$ tal que o conjunto $\left\{\beta \in \mathfrak{B}: W_{\beta} \cap V(y) \neq \emptyset\right\}$ é finito. Assim, $V(y) \cap Y$ é um aberto de $Y$ contendo $y$ tal que o conjunto

$$
\left\{\beta \in \mathfrak{B}:\left(W_{\beta} \cap Y\right) \cap(V(y) \cap Y) \neq \emptyset\right\}
$$

é finito e, portanto, $Y$ é paracompacto.

PROPOSIÇÃo A.3.10. Todo espaço paracompacto é normal. 
Demonstração: Seja $X$ um espaço paracompacto e mostremos primeiramente que $X$ é regular. Dados $a \in X$ e $F$ um fechado de $X$ tal que $a \notin F$, desde que $X$ é Hausdorff ${ }^{1}$, para cada $x \in F$, podemos escolher um aberto $U(x)$ que contém $x$ tal que $a \notin \bar{U}(x)$. A coleção

$$
\mathscr{U}=\{U(x): x \in F\} \cup\{X-F\}
$$

é uma cobertura aberta de $X$ e, sendo $X$ paracompacto, existe um refinamento aberto localmente finito $\mathscr{V}$ de $\mathscr{U}$. Consideremos a seguinte subcoleção

$$
\mathscr{W}=\{W \in \mathscr{V} ; W \cap F \neq \emptyset\} \subset \mathscr{V} .
$$

Então, $\mathscr{W}$ é uma cobertura aberta de $F$ e, além disso, se $W \in \mathscr{W}$, então $a \notin \bar{W}$. Desde que $W \cap F \neq \emptyset$, existe $U(x) \in \mathscr{U}$ tal que $W \subset U(x)$, $\operatorname{com} a \notin \bar{U}(x)$. Seja

$$
V=\bigcup_{W \in \mathscr{W}} W
$$

Temos que $V$ é um aberto de $X$ contendo $F$ e, desde que $\mathscr{W}$ é uma cobertura aberta localmente finita (pois $\mathscr{V}$ o é) segue de A.3.4 que

$$
\bar{V}=\bigcup_{W \in \mathscr{W}} \bar{W} .
$$

Assim, $a \notin \bar{V}$. Portanto, $V$ e $X-\bar{V}$ são abertos disjuntos contendo $F$ e $a$, respectivamente, o que implica que $X$ é regular.

Para concluirmos que $X$ é normal, sejam $F_{1}$ e $F_{2}$ dois fechados disjuntos de $X$. Usando a regularidade $\mathrm{e}^{2}$ de $X$, para cada $x \in F_{1}$ podemos escolher um aberto $U(x)$ de $X$ contendo $x$ tal que $\overline{U(x)} \cap F_{2}=\emptyset$. Usando os mesmos argumentos anteriores, é possível exibir um aberto $V$ de $X$ contendo $F_{1}$ tal que $\bar{V} \cap F_{2}=\emptyset$. Dessa forma, $V$ e $X-\bar{V}$ são abertos disjuntos contendo $F_{1}$ e $F_{2}$, respectivamente, o que implica que $X$ é normal.

obServaÇão A.3.11. Usando-se a Proposição A.3.10 e o Teorema de E.Michael [17, Pg.163, Teorema 2.3(2)], prova-se o resultado a seguir.

Proposição A.3.12. [17, Pg.165, Teorema 2.6] Sejam X um espaço paracompacto e $f: X \rightarrow Y$ uma aplicação contínua, fechada e sobrejetora. Então, $Y$ é paracompacto.

PROPOSIÇão A.3.13. O produto de um espaço paracompacto com um espaço compacto Hausdorffé paracompacto.

Demonstração: Sejam $X$ um espaço paracompacto e $Y$ um espaço compacto Hausdorff. Dada uma cobertura aberta $\mathscr{U}$ de $X \times Y$, para cada $x \in X$ fixado, desde que $\{x\} \times Y$ é compacto, existe uma subcoleção finita $\left\{U_{\alpha_{1}}^{x}, \ldots, U_{\alpha_{n_{x}}}^{x}\right\}$ de $\mathscr{U}$ que cobre $\{x\} \times Y$. Podemos

\footnotetext{
${ }^{1} X$ é Hausdorff se, e somente se, dado $p \in X$, para cada ponto $q \neq p$ em $X$, existe um aberto $U(p) \subset X$ tal que $q \notin \overline{U(p)}$ (Vide [17, Pg.138, Teorema 1.2]).

${ }^{2} X$ é regular se, e somente se, dados um ponto $x \in X$ e um fechado $F \subset X$ que não contém $x$, existe um aberto $V \subset X$ contendo $x$ tal que $\bar{V} \cap F=\emptyset$ Vide [17, Pg.141, Teorema 2.2].
} 
escolher um aberto $V(x) \subset X$ contendo $x$ tal que $V(x) \times Y \subset \bigcup_{i=1}^{n_{x}} U_{\alpha_{i}}{ }^{3}$. Consideremos a cobertura aberta

$$
\mathscr{U}_{X}=\{V(x): x \in X\}
$$

de $X$. Como $X$ é paracompacto, existe um refinamento aberto localmente finito $\mathscr{V}_{X}$ de $\mathscr{U}_{X}$ e assim, para cada $V \in \mathscr{V}_{X}, V \subset V(x)$ para algum $x \in X$. Mostremos que a cobertura aberta

$$
\mathscr{W}=\left\{(V \times Y) \cap U_{\alpha_{i}}^{x} ; i=1, \ldots, n_{x}, V \in \mathscr{V}_{X}\right\}
$$

de $X \times Y$ é um refinamento aberto localmente finito de $\mathscr{U}$. Com efeito, temos que $(V \times$ $Y) \cap U_{\alpha_{i}}^{x} \subset U_{\alpha_{i}}^{x}$, com $U_{\alpha_{i}}^{x} \in \mathscr{U}$. Além disso, dado $(x, y) \in X \times Y$, como $\mathscr{V}_{X}$ é localmente finito, existe um aberto $U$ de $X$ contendo $x$ tal que $U \cap V \neq \emptyset$, apenas para um número finito de elementos $V \in \mathscr{V}_{X}$. Então, considerando o aberto $U \times Y$ de $X \times Y$ contendo $(x, y)$, temos que $(U \times Y) \cap\left[(V \times Y) \cap U_{\alpha_{i}}^{x}\right] \neq \emptyset$, apenas para um número finito de elementos $(V \times Y) \cap U_{\alpha_{i}}^{x} \in \mathscr{W}$ e, portanto, $X \times Y$ é paracompacto.

DEFINIÇÃo A.3.14. Um par de espaços $(X, A)$ é chamado par paracompacto, se $X$ for paracompacto e se $A$ for um subespaço fechado de $X$ (Vide [4, Pg.7]).

OBSERVAÇÃo A.3.15. A categoria dos pares paracompactos $(X, A)$ e das aplicações de pares de espaços paracompactos $f:(X, A) \rightarrow(Y, B)$ será denotada por Top ${ }_{p c}^{2}$.

DEFINIÇão A.3.16. Um espaço topológico $X$ é chamado $\sigma$-compacto se ele pode ser escrito como uma reunião enumerável de subconjuntos compactos (Vide[38, Pg.833]).

obServaÇão A.3.17. Ernest Michael mostrou em [38, Proposição 4, Pg.837] que o produto cartesiano de um espaço paracompacto com um espaço regular $\sigma$-compacto é paracompacto.

\section{A.4 Join de Espaços Topológicos}

Para qualquer espaço topológico $X$, o cone $C X$ é o espaço quociente $\frac{(X \times I)}{\sim}$, onde $I=$ $[0,1]$ e $\sim$ é a relação de equivalência definida por $(x, 1) \sim\left(x^{\prime}, 1\right)$, para todo $x, x^{\prime} \in X$ ou, equivalentemente,

$$
C X=\frac{X \times I}{X \times\{1\}} .
$$

Intuitivamente, o cone $C X$ é obtido a partir de $X \times I$, identificando-se $X \times\{1\}$ em um único ponto e pode ser visto como sendo a união de todos os segmentos de reta ligando os pontos de $X$ a um ponto que não pertence a $X$, chamado vértice. De maneira análoga, a suspensão $S X$ é o espaço quociente $\frac{(X \times I)}{\sim}$, onde $\sim$ é a relação de equivalência $(x, 0) \sim\left(x^{\prime}, 0\right)$ e $(x, 1) \sim\left(x^{\prime}, 1\right)$, para todo $x, x^{\prime} \in X$. Intuitivamente, $S X$ é obtido de $X \times I$ identificando-se $X \times\{0\}$ a um vértice e $X \times\{1\}$ a um outro vértice e pode ser visto como sendo a união de todos os segmentos de reta ligando pontos de $X$ a dois vértices distintos, ambos não pertencendo a $X$. Mais geralmente, temos a seguinte

\footnotetext{
${ }^{3}$ Sejam $A \subset X, B \subset Y$ e $W$ um aberto em $X \times Y$ tal que $A \times B \subset W$. Se $A$ e $B$ são compactos, existem abertos $U \subset X$ e $V \subset Y$ tais que $A \times B \subset U \times V \subset W$ (Vide [42, Pg.171, Exercício 9])
} 
DEFINIÇÃo A.4.1. O join $X * Y$ de dois espaços $X$ e $Y$ é o espaço quociente

$$
X * Y=\frac{X \times I \times Y}{\sim}
$$

onde é a relação de equivalência definida por

$$
\begin{aligned}
& (x, 0, y) \sim\left(x, 0, y^{\prime}\right), \forall y, y^{\prime} \in Y \text { e para cada } x \in X \text { fixado, } \\
& (x, 1, y) \sim\left(x^{\prime}, 1, y\right), \forall x, x^{\prime} \in X \text { e para cada } y \in Y \text { fixado. }
\end{aligned}
$$

A classe de equivalência de um elemento $(x, t, y)$ em $X * Y$ será denotada por $[x, t, y]$. Observemos que o espaço $X$ torna-se naturalmente um subespaço do join $X * Y$, onde os pontos de $X$ são pontos finais de segmentos de reta. A inclusão natural é dada por

$$
\begin{aligned}
\imath: X & \hookrightarrow X * Y \\
x & \mapsto[x, 0, y] .
\end{aligned}
$$

Notemos também que a fórmula A.1 para a inclusão faz sentido e não depende da escolha particular do ponto $y \in Y$. Existe um mergulho similar

$$
\begin{aligned}
\jmath: Y & \hookrightarrow X * Y \\
y & \mapsto[x, 1, y] .
\end{aligned}
$$

Intuitivamente, $X * Y$ é obtido identificando-se o subespaço $X \times\{0\} \times Y$ a $X$ e o subespaço $X \times\{1\} \times Y$ é identificado com $Y$. Esse espaço pode ser visualizado como sendo a união de todos os segmentos de reta unindo pontos em $X$ a pontos em $Y$, onde tais segmentos não possuem pontos em comum, exceto possivelmente em seus extremos, ou seja, todo ponto $[x, y, t] \in X * Y$ está sobre um único segmento de reta unindo o ponto $x \in X \subset X * Y$ ao ponto $y \in Y \subset X * Y$; tal segmento é obtido fixando-se os pontos $x, y$ e variando-se a coordenada $t$.

EXEMPLO A.4.2. Seja $X$ um espaço topológico arbitrário.

(i) Se $Y=\left\{y_{0}\right\}$, então $X *\left\{y_{0}\right\}$ é o cone $C X=\frac{X \times I}{X \times\{1\}}$.

(ii) Se $Y=\left\{y_{1}, y_{2}\right\}$ for o espaço consistindo de dois pontos distintos, então $X * Y$ é a suspensão $S X$ discutida anteriormente. Observemos que a suspensão pode ser vista como a união de dois cones, com vértices $y_{1}$ e $y_{2}$ respectivamente, identificados ao longo do "equador" $X$, ou seja,

$$
\begin{aligned}
& \left(x, 0, y_{1}\right) \sim\left(x, 0, y_{2}\right), \text { para cada } x \in X \\
& \left(x, 1, y_{1}\right) \sim\left(x^{\prime}, 1, y_{1}\right) \text { e }\left(x, 1, y_{2}\right) \sim\left(x^{\prime}, 1, y_{2}\right), \text { para todo } x, x^{\prime} \in X .
\end{aligned}
$$

(iii) $S^{0} * S^{0}$ é homeomorfo à esfera $S^{1}$. Mais geralmente, $S^{n} * S^{m}$ é homeomorfo à esfera $S^{n+m+1}$, via o homeomorfismo $\left(t_{1} z_{1}, t_{2} z_{2}\right) \mapsto\left(\sqrt{t_{1}} z_{1}, \sqrt{t_{2}} z_{2}\right)$. Detahes desse fato no caso $n=1$ e $m=2 n-1$ podem ser encontrados em [2].

(iv) Se $X$ e $Y$ forem iguais ao intervalo fechado $I=[0,1]$, então $I \times I \times I$ é um cubo e $I * I$ é obtido identificando-se as duas faces opostas desse cubo: $X \times\{0\} \times Y$ e $X \times\{1\} \times Y$ aos segmentos de reta $X \times\{0\} \times\{0\}$ e $\{0\} \times\{1\} \times Y$, respectivamente e, deste modo, $I * I$ é homeomorfo a um tetraedro. 
LEMA A.4.3. As inclusões $\imath: X \hookrightarrow X * Y$ e $J: Y \hookrightarrow X * Y$ definidas em A.1 e A.2 respectivamente, são homotópicas a uma aplicação constante.

Demonstração: Fixemos um ponto $y_{0} \in Y$. Por definição, o mergulho $l: X \hookrightarrow X * Y$ se fatora como a composição

$$
\begin{aligned}
\imath: \quad X & \hookrightarrow X *\left\{y_{0}\right\} \subset X * Y \\
x & \mapsto\left[x, 0, y_{0}\right] .
\end{aligned}
$$

Mas, como observado no Exemplo A.4.2 (i), $X *\left\{y_{0}\right\}$ é o cone sobre $X$ e, portanto, contrátil. Isso significa que $\imath$ é homotópica à constante, como queríamos demonstrar. De maneira inteiramente análoga, mostra-se que a inclusão $J: Y \hookrightarrow X * Y$ é homotópica a uma aplicação constante.

Desde que todo ponto $[x, t, y] \in X * Y$ está sobre um único segmento de reta unindo o ponto $x \in X \subset X * Y$ ao ponto $y \in Y \subset X * Y$, uma forma conveniente de se escrever um elemento de $X * Y$ é como uma combinação linear formal $t_{1} x+t_{2} y$, com $0 \leq t_{i} \leq 1 \mathrm{e}$ $t_{1}+t_{2}=1$, com as seguintes condições: $0 x+1 y=y, \forall x \in X$ e $1 x+0 y=x, \forall y \in Y$, as quais correspondem exatamente às identificações $(x, 1, y) \sim\left(x^{\prime}, 1, y\right) \mathrm{e}(x, 0, y) \sim\left(x, 0, y^{\prime}\right)$, respectivamante, na definição de $X * Y$. De maneira análoga, o join de uma coleção finita de espaços topológicos $\left\{X_{1}, X_{2}, \ldots, X_{n}\right\}$ é definido como segue.

DEFINIÇão A.4.4. O join $X_{1} * \cdots * X_{n}$ de $n$-espaços $X_{1}, \ldots, X_{n}$ é o espaço das combinações lineares formais $t_{1} x_{1}+\cdots+t_{n} x_{n}$ tais que $0 \leq t_{i} \leq 1$ e $\sum_{i=1}^{n} t_{i}=1$, com a convenção de que os termos $0 t_{i}$ podem ser omitidos.

EXEMPlo A.4.5. Um caso especial da definição acima é quando cada um dos espaços $X_{i}$ é apenas um ponto. Assim, temos que:

(i) $\mathrm{O}$ join de dois pontos $\left\{x_{0}\right\} *\left\{x_{1}\right\}$ é um segmento de reta.

(ii) $\mathrm{O}$ join de três pontos $\left\{x_{0}\right\} *\left\{x_{1}\right\} *\left\{x_{2}\right\}$ é um 2-simplexo com vértices $x_{0}, x_{1}, x_{2}$.

(iii) $\mathrm{O}$ join de quatro pontos $\left\{x_{0}\right\} *\left\{x_{1}\right\} *\left\{x_{2}\right\} *\left\{x_{3}\right\}$ é um 3 -simplexo com vértices $x_{0}, x_{1}, x_{2}, x_{3}$.

(iii) Mais geralmente, se $\left\{x_{0}, x_{1}, \ldots, x_{k}\right\}$ for uma coleção de $k+1$ pontos distintos, então, o join $x_{0} * x_{1} * \ldots * x_{k}$ é a envoltória convexa desses pontos e, portanto, é o $k$-dimensional simplexo $\Delta^{k}$ com vértices $\left\{x_{0}, x_{1}, \ldots, x_{k}\right\}$. Se os $k+1$ pontos $\left\{x_{0}, x_{1}, \ldots, x_{k}\right\}$ são tais que $\left\{x_{i}-x_{0}, i=1, \ldots, k\right\}$ formam a base canônica do $\mathbb{R}^{k}$, então o seu join é o $k$-simplexo padrão

$$
\Delta^{k}=\left\{\left(t_{0}, \ldots, t_{k}\right) \in \mathbb{R}^{k+1} ; \sum_{i=0}^{k} t_{i}=1 \text { e } 0 \leq t_{i} \leq 1\right\} .
$$

Mais geralmente, o join ${ }^{4}$ de uma família arbitrária de espaços topológicos pode ser definido como segue

\footnotetext{
${ }^{4}$ Essa construção é devida a Milnor [39].
} 
DEFINIÇão A.4.6. Dada uma família de espaços topológicos $\left\{X_{j}\right\}_{j \in J}$, definimos o join $X=$ $*_{j \in J} X_{j}$ como sendo o seguinte espaço: os elementos de $X$ são representados por $J$-uplas

$$
\left(t_{j} x_{j}\right)_{j \in J} \text {, onde } t_{j} \in[0,1], x_{j} \in X_{j} \text { e } \sum_{j \in J} t_{j}=1 \text {, }
$$

onde apenas um número finito dos $t_{j^{\prime} s}$ são não nulos. Os elementos $\left(t_{j} x_{j}\right)$ e $\left(u_{j} y_{j}\right)$ representam o mesmo elemento de $X$ se, e somente se,

(i) para cada $j \in J, t_{j}=u_{j}$,

(ii) para cada $j \in J, t_{j} \neq 0$, implica que $x_{j}=y_{j}$.

Temos aplicações coordenadas

$$
\begin{aligned}
t_{j}: \quad X=*_{j \in J} X_{j} & \rightarrow[0,1] \\
\left(t_{i} x_{i}\right) & \mapsto t_{j} \\
p_{j}: \quad t_{j}^{-1}(0,1] & \rightarrow X_{j} \\
\left(t_{i} x_{i}\right) & \mapsto x_{j} .
\end{aligned}
$$

A topologia de Milnor sobre $X=*_{j \in J} X_{j}$ é a topologia menos fina que torna contínuas todas as aplicações $t_{j}$ e $p_{j}$, a qual pode ser caracterizada pela seguinte propriedade universal: uma aplicação $f: Y \rightarrow X=*_{j \in J} X_{j}$ é contínua se, e somente se, as aplicações

$$
t_{j} \circ f: Y \rightarrow[0,1] \quad \text { e } \quad p_{j} \circ f: f^{-1}\left(t_{j}^{-1}(0,1]\right) \rightarrow X_{j}
$$

são contínuas, onde $Y$ é arbitrário.

Proposição A.4.7. $O$ join $X_{1} * \cdots * X_{n}$ de $n$-espaços topológicos compactos é um espaço compacto.

Demonstração: É suficiente provar o caso $n=2$ e o resultado seguirá por indução. Dados $X_{1}, X_{2}$ espaços compactos, o produto cartesiano $X_{1} \times I \times X_{2}$ é um espaço compacto. Desde que a projeção

$$
p: X_{1} \times I \times X_{2} \rightarrow X_{1} * X_{2}=\frac{X_{1} \times I \times X_{2}}{\sim}
$$

é contínua e sobrejetora, segue que $X_{1} * X_{2}$ é compacto. 


\section{Referências Bibliográficas}

[1] Atiyah, M.F. and MacDonald, I.G., Introduction to Commutative Algebra. AddisonWesley, Reading, MA (1972)

[2] Araújo, A.M., Versão Homológica do Teorema de Borsuk-Ulam para funções $\mathbb{Z}_{q^{-}}$ equivariantes, Dissertação de Mestrado, UFSCAR (1998).

[3] Baues, H.J., The Algebra of Secondary Cohomology Operations Progress in Mathematics 2003

[4] Bacon, P., Axioms for the Čech Cohomology of Paracompacta Pacific Journal of Mathematics, Vol. 52, $N^{o} 1$, (1974).

[5] Borel, A., Seminar on Transformation Groups. Ann. of Math. Studies 46 Princeton Univ. Press Princenton N.J., 1960.

[6] Borsuk, K.,Uber Zerlegung einer euklidischen n-dimensionalen Volkugeln in $n$ Mengen Verhandlungen des Internationalen Mathematiker Kongresses, Zürich, (1932); II. Band: Sekt.-Vorträge, Orel Fussli, Zürich, (1932), 142-198.

[7] Borsuk, K.,Drei Sätze über die n-dimensionale euklidische Sphäre. Fund. Math. 20 (1933), 177-190.

[8] Bredon, G.E., Introduction to Compact Transformation Groups, Pure and Applied Mathematics 46 Academic Press New York and London, (1972).

[9] Brouwer, L.E., Über Abbildung von Mannigfaltigkeiten, Mathematische Annalen, 71(4)(1912), 598.

[10] Dieck, T., Transformation Groups, Walter de Gruyter, Berlin-New York, (1987).

[11] Dieck, T.t. and Bröcker, T., Representations of Compact Lie Groups, Springer-Verlag, New-York, Berlin, Heidelberg, Tokio, 1985. 
[12] Dieck, T.t., Algebraic Topology, EMS Textbooks in Mathematics, European Mathematical Society, 2008.

[13] Dold, A., Lectures on Algebraic Topology, Second Edition, Springer-Verlag, Berlin, Heidelberg, New York, (1980).

[14] Dowker, C.H., Čech Cohomology Theory and the Axioms, Annals of Mathematics, Vol. 51, no. 2, 1950.

[15] Conner, P.E. and Floyd, E.E, Fixed point free involutions and equivariant maps, Bull. Amer. Math. Soc. (N.S.) 66 (1960), 416-441.

[16] Conner, P.E. and Floyd, E.E, Fixed point free involutions and equivariant maps II, Trans. Amer. Math. Soc. 105 (1962), 222-228.

[17] Dugundji, J., Topology, Allyn and Bacon, Inc.,Boston, (1966).

[18] Gonçalves, D.L., Jaworowski J., Pergher, P.L.Q. and Volovikov, A.Y. Coincidences for maps of spaces with finite group actions. Topology and its Appl. 145 n.1-3 61-68, 2004.

[19] Eilenberg, S.,On the theorem of P.A. Smith concerning fixed points for periodic maps. Duke Mathematical Journal 6 (1940), 428-437.

[20] Fadell, E.R. and Huseini, S., An ideal-valued cohomological index theory with applications to Borsuk-Ulam and Bourgin-Yang theorems. Ergodic Theory Dynam. Systems 8 (1988), Charles Conley Memorial Issue, 73-85.

[21] Fadell, E.R. and Husseini, S., Relative cohomological index theories Adv. in Math. 64:1 (1987), 1-31.

[22] Fadell, E.R. and Rabinowitz, Generalized cohomological index theories for Lie groups actions with an application to bifucartion questions for Hamiltonian systems, Invent. Math. 45:2 (1978), 139-174.

[23] Gottlieb, D.H. Fibre bundles and the Euler characteristic. Journal Differential Geom. 10 (1975), 39-48.

[24] Hara, Y. The degree of equivariant maps, Topology and its Applications, 148, (2005), Pg. 113-121.

[25] Hatcher, A. Algebraic Topology, Cambridge University Press 2002

[26] Hofmann, K.H. and Morris, S.A., The Structure of Compact Groups, 2nd Revised and Augmented Edition, Berlin-New York, Walter de Gruyter, 2006.

[27] Howard, P., Keremedis, K., Rubin, H. and Rubin, J., Disjoint Unions of Topological Spaces and Choice, Math. Log. Quart. Vol.44 (1998), 493-508.

[28] Hu, S.T. Introduction to Homological Algebra, Holden-Day, Inc., San Francisco, Cambridge, London, Amsterdam, (1968) 
[29] Hungerford, T.W., Algebra, Springer-Verlag, New York, (1974).

[30] Husemoller, D., Fibre Bundles, Third edition, Springer-Verlag, New York (1994).

[31] Jaworowski, J. Maps of Stiefel Manifolds and a Borsuk-Ulam Theorem. Proc. Edinburg Math. Soc. 98 (1986), 158-162.

[32] Jaworowski, J. The degree of maps of free G-manifolds and the average. Journal Fixed Point Theory appl. 1 (2007), 111-121.

[33] Komiya, K. Borsuk-Ulam Theorem and Stiefel Manifolds. Osaka J. Math. 43 (2006), 183-191.

[34] Lima, E.L., Variedades Diferenciáveis, Publicações Matemáticas, Impa, (2008).

[35] Lusternik, L. and Schnirelman, A.,Métodes Topologiques dans des Problèmes Variationels. Hermann, 1934.

[36] May, P. A Concise Course in Algebraic Topology, Chicago Lectures in Mathematics Serie, The Uiversity of Chicago Press, (1999).

[37] McCleary, J. User's Guide to Spectral Sequences. Mathematics Lectures Series Publish or Perish, Inc., Wilmington, Delaware U.S.A., 1985.

[38] Michael, E., A Note on Paracompact Spaces, Proceedings of the American Mathematical Society Vol. 4, n.5 (1953), 831-838.

[39] Milnor, J., Construction of Universal Bundles. II, Ann. of Math. 63 (1956), 430-436.

[40] Mimura, M. and Toda, H., Topology of Lie Groups, I and II, American Mathematical Society, Providence, Rhode Island, Vol.91, (1991).

[41] Montgomery, D., Orbits of highest dimension, Seminar on TRansformation Groups, Ann. of Math. Studies, No. 46, Chapter IX. Princeton Univ. Press, Princeton, New Jersey, 1960.

[42] Munkres, J.R., Topology, A First Course, Prentice Hall, 2000.

[43] Munkres, J.R., Elements of Algebraic Topology, The Benjamin/Cummings Publishing Company, Inc., Cambridge, Massachusetts, (1984).

[44] Pergher, P.L.Q., de Mattos, D. and dos Santos, E.L. The Borsuk-Ulam Theorem for General Spaces. Arch. Math. 81 n.1 96-102, 2003.

[45] Pontrjagin, L., Topological Groups, Princeton University Press, Princeton, 1946.

[46] Rotman, J., An Introduction to the Theory of Groups Graduate Texts in Mathematics 148 ((4th ed.) ed.). Springer-Verlag.

[47] Shvarts, A.S., Some estimates of the genus of a topological space in the sense of Krasnosel'ski , Uspekhi Mat. Nauk 12:4 (1957), 209-214 (Russian). 
[48] Shvarts, A.S., The genus of a fiber space, Trudy Moskov. Mat. Obshch. 11 (1962), 99126; English translate in Amer. Math. Soc. Transl.(2)55 (1966).

[49] Spanier, E.H., Algebraic Topology, Springer-Verlag, New York, 1966.

[50] Steenrod, N., The Topology of Fiber Bundles, Princeton Mathematical Series No. 14, Princeton University Press, (1951).

[51] Vassiliev, V.A. Introduction to Topology American Mathematical society 2001

[52] Vick, J.W. An Introduction to Algebaric Topology Second Edition, Springer Verlag, (1994).

[53] Wallace, A.H., Algebraic Topology, Homology and Cohomology W. A. Benjamin Inc., New York, (1970).

[54] Yang, C.T., On theorems of Borsuk-Ulam, Kakutani-Yamabe-Yujobo and Dyson. I, Ann. of Math. 60:2 (1954), 262-282.

[55] Yang, C.T., On theorems of Borsuk-Ulam, Kakutani-Yamabe-Yujobo and Dyson. II, Ann. of Math. 62:2 (1955), 271-283. 


\section{Índice Remissivo}

$G$-índice valor ideal absoluto, 57

$G$-ação à direita, 27

$G$-espaço, 28

$G$-fibrado

principal, 32

produto, 33

universal, 34

$G$-variedade, 27

$R$-módulo

de cohomologia orientado, 11

graduado, 49

de $q$-cadeias simpliciais relativo, 10

de $q$-cocadeias simpliciais relativo, 11

livre, 9

Índice

de Fadell e Rabinowitz, 66

de valor ideal relativo, 53

de valor numérico absoluto, 58

de valor numérico relativo, 53

de Yang, 66

órbita, 28

Ação

à esquerda, 26

diagonal, 30

efetiva, 29

Livre, 29

produto, 27

Transitiva, 29
Trivial, 29

Aplicação

$G$-homotopia, 32

$G$-equivariante, 29

classificante, 35

de órbitas, 28

de Borel, 40

de pares, 83

de pares de Borel, 40

induzida nos espaços órbitas, 31

induzida nos espaços de órbitas, 30

multiplicação:inversão, 24

projeção natural, 28

de pares $G$-equivariante, 31

de pares simpliciais, 9

própria, 30

projeção, 13

simplicial, 8

Axiomas

de Eilenberg-Steenrod, 19

Caregoria, 83

Cobertura

cobertura aberta do par, 11

localmente finita, 85

nervo de uma cobertura, 13

refinamento, 85

refinamento, 12

Cohomologia

de Čech, 14 
Complexo

de cocadeias, 11

simplicial abstrato, 7

subcomplexo, 8

Conjunto

cofinal, 4

dirigido, 1

pré-ordenado, 1

Construção de Milnor, 37

Construção de Milnor, 37

Continuidade

Čech, 20

equivariante, 47

Espaço

$G$-espaço à esquerda, 27

$G$-invariante, 29

Borel, 39

classificante, 34

paracompacto, 85

Fibrado

$G$-fibrado principal, 32

$G$-fibrado universal, 34

induzido, 33

produto, 33

filtração decrescente, 75

Funtor

cohomologia Čech, 18

cohomologia equivariante Borel, 45

contravariante, 85

covariante, 84

construção de Borel, 41

Graduado

anel, 49

Grupo

$G L(n, \mathbb{R}), 26$

$O(n), 26$

$S L(n, \mathbb{R}), 26$

compacto, 24

de Lie, 25

topológico, 24

grupo de transformação, 27
Homomorfismo

de $R$-módulos graduados, 50

induzido em cohomologia $G$-equivariante, 45

transfer, 72,78

induzido em cohomologia de Čech, 17

Homotopia

de pares, 83

Ideal

graduado, 53

Integração ao longo fibra, 76

Join

de espaços topológicos, 89

de uma família de espaços topológicos,

91

Limite

direto de $R$-homomorfismos, 3

direto de $R$-módulos, 2

direto do sistema direto de espaços topológicos, 6

módulo diferencial, 73

Morfismo

$B$-morfismo, 34

Operador

cobordo relativo, 11

bordo, 10

Par

$G$-par paracompacto, 32

$G$-par, 31

de Borel, 40

de espaços, 83

simplicial, 9

Produto cup

$G$-equivariante, 47

Čech, 21

Pull-back, 33

Sequência espectral, 74

Simplexo

$q$-simplexo, 7

orientado, 9 
Sistema

direto, 1

direto de $R$-módulos, 2

direto de espaços topológicos, 6

soma topológica, 6

subgrupo de isotropia, 28

Translação à esquerda, 27

União

disjunta, 6

livre, 6

Variedade

$G$-variedade, 31

de Stiefel, 67

Grassmanniana, 68

Vizinhança

par de vizinhanças fechadas, 20

par de vizinhanças, 20 